\title{
Abstracts \\ www.anatomy.org.tr \\ doi:10.2399/ana.15.001s \\ Abstracts for the \\ 13th Turkish Neuroscience Congress April 30th 2015 to May 3rd 2015, Konya, Turkey
}

\section{Invited Lectures and Conferences}

$$
\text { (C-001 - C-52) }
$$

Thursday, 30 April 2015

$17: 30-18: 30$

OPENING LECTURE (CONFERENCE 1)

New perspectives in neurosciences

Chairs: Prof. Dr. Nuran Hariri \& Prof. Dr. Serdar Gergerlioğlu

\section{C-001}

\section{New perspectives in neurosciences}

Yaşargil G

Department of Neurosurgery, Yeditepe University, Faculty of Medicine, Istanbul, Turkey

Intensive research activities within the past 200 years have revealed that the central nervous system (CNS) is a heterogeneous, heteromorphic, multidimensional and multifunctional compound organ system. Scientists working in Comparative Anatomy and Embryology determined that the transformativeadaptive evolution of the CNS occurred over an extensive time period of 500 million years and continues to unfold and progress. The phylogenic, ontogenic, histogenic, angiogenic, endocrinogenic and immunogenic defined compartments of the CNS present a unique macro- and microarchitecture along the spinal cord, brain stem, diencephalon and telencephalon with archipallial, paleopallial and neopallial intertwining architecture. The continuing upswing in scientific technology is providing medicine and surgery with tools of immense value; the availability of achromatic microscope, free from spherical aberration, invented by J.J. LISTER, 1830, in the United Kingdom, later the formulation of the equation "Angular Aperture" by Ernst ABBE, in 1880, Zeiss Company, Jena, Germany, followed by the Electron Microscope in 1933, Scanning Tunneling Microscope in 1981 and Positron Microscope in 1987. Scientists in biology and neurobiology have thus been compelled and stimulated to initiate profound studies on morphology and function of all organs and their cells.
Scientists in molecular biology and genetics were ultimately able to reveal a fascinating inter- and intracellular microarchitecture such as receptors, microtubules, mitochondria and synapses. Scientists in physics and chemistry finally succeeded to bypass Abbe's optical limitation of 0.2 micrometers and the nanoscope was born. This latter achievement opens a new era in biology and pathology, allowing the study in-vivo of ultrastructures. The visualization of living ultra- micro-structures is a great accomplishment, a culmination of centuries of scientific endeavors.In contrast, the phylogenic aspects of vascular, neoplastic, degenerative, toxic and viral diseases of the CNS had hitherto found only marginal attention. The clinical, neuroradiological and neuropathological observations on a large number of patients with neurovascular and neoplastic lesions, degenerative diseases and viral infections alerted us to specific predilection sites of these diseases, which affect certain compartments of the CNS, in relation to their phylogenic, ontogenic, histogenetic and angiogenic order. The adjacent compartments remain unaffected. The conduct of these lesions teaches us new aspects in neuropathology. These observations will be presented and discussed.

Friday, 1 May 2015

$8: 30-10: 30$

SYMPOSIUM 1

Neuroendocrine control of reproduction

Chairs: Prof. Dr. Haluk Keleştimur \& Doç. Dr. Süleyman Sandal

\section{C-002}

Neuroendocrine control of hypothalamus-pituitarygonadal axis

Keleştimur H

Department of Physiology, FIrat University Medical School, Elazığ, Turkey 
Gonadotropin-releasing hormone $(\mathrm{GnRH})$ is a hypothalamic neuropeptide that regulates the release of follicular-stimulating hormone (FSH) and luteinizing hormone (LH) from the anterior pituitary. These gonadotropic hormones lead to gonadal growth and production of gonadal sex steroids, eliciting the development of secondary sexual characteristics. The pulsatile pattern of gonadotropin-releasing hormone release has a critical importance in the intermittent release of these gonadotropic hormones from the pituitary. Unlike the other hypothalamic cell groups, GnRH neurons originate from the olfactory placode region to the hypothalamus during embryogenesis. The activation of these neurons occurs during juvenile development, yet initiates puberty via their reactivation. However, the mechanisms underlying this reactivation are not well understood. During the last decade, our understanding of this hypothalamus-pituitarygonadal axis has quickly expanded. Two novel hypothalamic ArgPhe-amide- related peptides, kisspeptin and gonadotropininhibitory hormone $(\mathrm{GnIH})$, may be important regulators of the reproductive axis. Kisspeptin neurons of the arcuate nucleus (ARC) of the hypothalamus have been proposed as a critical component regulating the pulsatile release of GnRH, and its modulation by endogenous steroid hormones and exogenous environmental cues. GnIH may also directly modulate GnRH secretion because $\mathrm{GnIH}$-immunoreactive neurons have been shown to form close appositions with $\mathrm{GnRH}$ neurons. $\mathrm{GnIH}$ have inhibitory actions on GnRH neurons. In summary, kisspeptin and $\mathrm{GnIH}$ appear to be involved in GnRH secretion. These findings may have translational importance for understanding the basis for human disorders in which central control of $\mathrm{GnRH}$ secretion is impaired.

This study was supported by TUBITAK Project No:113S193.

Keywords: GnRH, kisspeptin, GnIH

\section{C-003}

\section{Electrophysiological features of neuroendocrine cells}

Ayar A

Department of Physiology, Karadeniz Technical University, Faculty of Medicine, Trabzon, Turkey

Neuroendocrine cells, so called because they are structurally similar to neurons and they secrete hormones like endocrine cells, are present throughout the body and constitute the diffuse network of neuroendocrine system. In accordance with their wide distribution they play crucial roles in variety of physiological bodily functions by responding to stimuli and their secretion products. In addition to their physiological and pathophysiological functions, neuroendocrine cells can sometimes, like other types of cells in our body, develop into cancer and lead to serious medical conditions, including neuroendocrine tumors. Use of electrophysiological techniques is a useful tool for investigating the neuroendocrine cells primary functions, namely controlled secretion of neurotransmitters and hormones, at single cell and subcellular level. Indeed identification of electrophysiological characteristics of certain neuroendocrine cells have not only significantly contributed to the clarification of control mechanism of neurotransmitters and hormones but also to understanding of related pathophysiological processes that are clinically relevant. Neuroendocrine system performs a vast variety of functions with ubiquitous neuroendocrine cell types including anterior pituitary cells, hypothalamic supraoptic and paraventricular neurosecretory cells, pancreatic islet cells, parathyroid cells, chromaffin cells, Sertoli cells, renin producing cells of the kidney, atrial natriuretic peptide producing cells of the heart, $\mathrm{G}$ cells of the gastric antrum, the gastrointestinal enterochromaffin cells and Merkel cells of the skin. The neuroendocrine cells share the characteristics of excitable cells as electrical activity plays important role in intercellular communication in the stimulus-secretion coupling a process involves, action potentials, cell surface receptors, voltage and gated ion channels, intracellular signaling (including intracellular calcium signaling). Utilization of electrophysiological techniques in the relatively new research field of neuroendocrinology have a great potential of clarification of physiological and pathophysiological roles of neuroendocrine cells and these information have a great potential to be translated from bench to hospital bed.

Activities on electrophysiological characteristics of neuroendocrine cells are supported by TUBITAK (proje No: 114S179).

\section{C-004}

\section{Calcium signaling in hypothalamus neurons}

\section{Özcan $\mathrm{M}^{1}$, Keleştimur $\mathrm{H}^{2}$, Ayar $\mathrm{A}^{3}$, Canpolat $\mathrm{S}^{2}$}

'Department of Biophysics, Firat University, Faculty of Medicine, Elazı̆g, Turkey; 'Department of Physiology, Firat University, Faculty of Medicine, Elazığ, Turkey; ${ }^{3}$ Department of Physiology, Karadeniz Technical University, Trabzon, Turkey

The pulsatile pattern of gonadotropin releasing hormone $(\mathrm{GnRH})$ release from hypothalamus, which results in the intermittent release of gonadotropic hormones from the pituitary, has a critical importance for reproductive function but the factors responsible from this release pattern are not known. A rise in intracellular free calcium concentration $([\mathrm{Ca} 2+] \mathrm{i})$ is the common trigger for exocytosis in most cell types. There are some agents which regulate $\mathrm{GnRH}$ secretion from the hypothalamus by changing $[\mathrm{Ca} 2+]$ i. The effects of kisspeptin, melatonin, leptin, orexin, alfa-MSH and NPY on [Ca2+]i were investigated in GT1-7 cells, an in vitro model of GnRH-secreting neurons. Kisspeptin-10 caused a triphasic change characterized by an initial small increase followed by a significant decrease and increase in $[\mathrm{Ca} 2+]$ i. Melatonin caused increase in intracellular $\mathrm{Ca} 2+$ transients in GT1-7 cells in a dose-dependent manner. Similarly orexin caused a significant increase in $[\mathrm{Ca} 2+]$ i compared to basal levels in GT1-7 cells. Leptin caused an increase in [Ca2+]i which is localised to certain sites of GT1-7 cells rather than being widespread and can not be detected significantly when the whole cell area is measured. Alfa-MSH and NPY did not have any significant effects on $[\mathrm{Ca} 2+] \mathrm{i}$ in GT1-7 cells. The results suggest that agents may exert their effects on $\mathrm{GnRH}$ release by producing different calcium responses in $[\mathrm{Ca} 2+]$.

This study was supported by TUBITAK Project No: 113 S193

Keywords: hypothalamus, calcium, GnRH, neuron 


\section{C-005}

Effects on reproductive function of antidepressants

Canpolat $\mathrm{S}$

Department of Physiology, Firat University, Faculty of Medicine, Elazı̆̆, Turkey

Sexual dysfunctions are among the most frequent adverse effects of selective serotonin uptake inhibitors (SSRI) that are the most preferred drugs used in treatment of depression. Dopamine and norepinephrine reuptake inhibitor antideprassants are less commonly associated with sexual dysfunctions, and also their beneficial effects have been reported in eliminating adverse effects of SSRI. There are a few studies regarding sexual effects of agomelatine, a new antidepressant that has antagonist actions at serotonergic 5HT2C receptors and agonist actions at melatonergic receptors. Side effects of antidepressants that are increasingly used in children are not well known. In the present study, effects of paroxetine (a SSRI type antidepressant) and bupropion (dopamine and norepinephrine reuptake inhibitor type antidepressant) and agomelatine on pubertal maturation were investigated in rat models. Paroxetine and agomelatine advanced puberty in the female rats. Puberty onset was delayed by bupropion and agomelatine in male rats. Cauda epididymal sperm numbers (106/organ) were significantly lower in the paroxetine, bupropion and agomelatine-treated groups. The present findings suggest that antideprassants may affect pubertal maturation differently in rats.

This study was supported by TUBITAK Project No: 113S193

Keywords: antidepressant, reproductive, paroxetine, agomelatine, bupropion

Friday, 1 May 2015

$8: 30-10: 30$

SYMPOSIUM 2

Neurogenesis and neuronal survival pathways in learning and memory

Chairs:: Prof. Dr. Işıl Kurnaz \& Prof. Dr. Cem Süer

\section{C-006}

The relation of ETS proteins with neuronal survival, neurogenesis and learning

Kurnaz Aksan I

Department of Molecular Biology and Genetics, Molecular Neurobiology Lab (Axanlab), Gebze Technical University, Kocaeli, Turkey

Elk-1, a member of the TCF (Ternary Complex Factor) subfamily of the ETS (E26) domain superfamily, is a ubiquitously expressed transcription factor. Yet, a mild mental retardation was reported in Elk-1 knockout mice, and in brain sections this transcription factor was found to be localized to dendrites and axons. Our and others' work have pointed out to a potential neuronal survival role for Elk-1. The purpose of this study has been to elucidate how Elk-1 may contribute to neuroprotection during hypoxic and normoxic conditions. In this study, we have first addressed in SH-SY5Y neuronal model systems whether Elk-1 localization and phosphorylation is affected by hypoxia vs normoxia using immunofluorescence. Subsequently, the genes regulated by Elk-1 under both conditions were analyzed using RTPCR and microarray analyses. Although there are minute changes in Elk-1 localization and phosphorylation, the more interesting result for us has been the genes regulated by Elk-1VP16 under even normoxic conditions; these were genes related to hypoxia, pluripotence and miRNA regulation. A previous preliminary study with CD133+ hNSC and hBTIC celsl had shown higher levels of Elk-1 expression (Singh lab, unpublished data). The fact that in microarray analysis Elk-1 was found to regulate pluripotency- and hypoxia-related genes, taken together with the preliminary hNSC data, suggests that especially in highly proliferating and thus transiently hypoxic cells Elk-1 might support survival. The relation of this to learning is an additional topic of interest, particularly since Elk-1 phosphorylation was shown to increase in learning and memory paradigms.

This work has been supported through TÜBİTAK grant no. $211 \mathrm{~T} 167$.

Keywords: Elk-1, hypoxia, neurogenesis, learning

\section{C-007}

Effect of thyroid hormone on intracellular signaling pathways involved in learning and behavior

Süer C

Department of Physiology, Medical Faculty of Erciyes University, Kayseri, Turkey

Rats are able to learn an initial location of hidden platform in a maze (initial learning) and then to "forget" this information and to learn a new location (reversal learning). This type of behavior requires for the experience-dependent enhancement and weakening of synaptic efficacy in the hippocampus, and are closely associated with LTP (long-term potentiation)- and LTD (longterm depression)-like mechanisms, which are both represent cellular correlates of bidirectional plasticity. Although the maximal vulnerability of the central nervous system to thyroid hormones (TH, T4 and T3) imbalance occurs during the earliest stages of development, synaptic plasticity can be also affected by moderate reductions/elevations in TH levels during adulthood. Actions of $\mathrm{TH}$ do not primarily involve nuclear $\mathrm{TH}$ receptors and thus are "nongenomic". Binding of T4 and-less potently, T3 to a cell surface receptor activates mitogen-activated protein kinase (MAPKERK1/2) pathway, leading to nuclear translocation of phosphoMAPK. Two important downstream molecules of this pathway are Elk-1 and CREB, having a pivotal role in the establishment of long-term memory. In agreement with previous studies, our data has shown that both hypothyroid and hyperthyroid states elicit similar effects on learning performance and LTP/LTD. This apparent paradox may be due to hypo- and hyperthyroidism activating distinct mechanisms that have similar end results. In the light of our data, we propose a hypothetical model for regulation of creb gene expression by $\mathrm{TH}$ receptors and for phosphorylation of CREB and Elk-1 by T4. This model may have clinically important results to explain cognitive decline in TH imbalances. 
This research was financially supported by the Scientific and Technological Research Council of Turkey (TUBITAK) grant number $113 \mathrm{~S} 345$ to C.S. This work was further supported by the Erciyes University Research Found grant number TOA-20134555 .

Keywords: thyroid hormone, learning and memory, longterm potentiation, long-term depression, intracellular signaling pathways

\section{C-008}

Synaptic plasticity, adult neurogenesis and memory

Laroche S

CNRS and University Paris, Sud, Paris and Saclay Neuroscience Institute, Orsay, France

A defining characteristic of the brain is its remarkable capacity to undergo activity-dependent functional and structural remodelling via mechanisms of plasticity that form the basis of our capacity to encode and retain memories. The prevailing model of how our brain stores new information suggests it resides in activitydriven modifications of synaptic strength and remodelling of neural networks brought about by cellular and molecular changes within the neurons activated during learning. To date, the idea that a form of activity-dependent synaptic plasticity known as long-term potentiation plays a central role in the laying down of memories has received considerable support. Beyond this mechanism of plasticity at the synapse, adult neurogenesis, the birth and growth of new neurons, is another form of neural plasticity that occurs continuously in defined brain regions such as the dentate gyrus of the hippocampus and there is accumulating evidence that this form of neural plasticity also contributes to memory function. Based on work on the role of the transcription factor Zif268, I will review recent evidence which support the idea that the occurrence of synaptic plasticity and the functional recruitment of newborn neurons in the hippocampus are functionally linked mechanisms of brain plasticity that are essential to store memories.

Keywords: learning, memory, synaptic plasticity, Zif268

Friday, 1 May 2015

11:00-11:45

\section{CONFERENCE 2}

What happens in your cerebellum while you learn a motor skill Chair: Prof. Dr. Ertuğrul Kılıç

\section{C-009}

\section{What happens in your cerebellum while you learn a} motor skill?

Lisberger $\mathrm{S}$

Duke University School of Medicine, Howard Hughes Medical Institute, Department of Neurobiology Investigator, Durham, NC, USA

The theory of cerebellar learning relies on motor errors signaled by climbing-fiber inputs to cause long-term depression of synaps- es from parallel fibers to Purkinje cells. In my talk, I will describe how we have transformed the cerebellar learning theory into a cerebellar learning reality. I will show that a climbing fiber input on one behavioral trial is linked to a depression of Purkinje cell output on the following trial and that plasticity is graded through modulation of the duration of the climbing fiber inputs. I will argue that we are observing the product of short-term cellular plasticity caused by the climbing fiber input. I will also show that short-term plasticity and/or learning strongly facilitate long-term learning. I will conclude that the climbing fiber input to the cerebellum plays a primary role in motor learning.

Friday, 1 May 2015

11:45-12:30

CONFERENCE 3

Nanomedicines for stroke

Chair: Prof. Dr. İsmail Hakkı Ulus

\section{C-010}

\section{Nanomedicines for stroke}

Dalkara T

Institute of Neurological Sciences and Psychiatry, Hacettepe University, Ankara, Turkey

Most of the potential therapeutic agents developed to treat CNS diseases have limited penetration to the brain. For example, caspase inhibitors (e.g. Z-DEVD-FMK) or growth factors (e.g. bFGF) suppress cell death, making them promising agents for treatment of the neurodegenerative diseases, but they cannot cross the blood-brain barrier (BBB). For this, we have developed brain-targeted chitosan nanoparticles to efficiently transport these peptides across the BBB. On the other hand, many neuroprotective drugs like adenosine, are inefficient on systemic administration because of their rapid clearance from the blood. For this, we have developed nanoassemblies by conjugating adenosine to squalene to obtain a prolonged circulation time. These nanoassembilies and nanoparticles provides excellent neuroprotection in a mouse stroke model.

Friday, 1 May 2015

$11: 45-12: 30$

CONFERENCE 4

Optogenetic dissection of feeding circuits

Chair: Prof. Dr. Ahmet Hacımüftüoğlu

\section{C-011}

Optogenetic dissection of feeding circuits

Atasoy D

Department of Physiology, Istanbul Medipol University, Istanbul, Turkey

New tools for mapping and manipulating molecularly defined neural circuits have improved the understanding of how the central nervous system regulates appetite. Activation of starvationsensitive AGRP neurons can rapidly elicit behavioral state similar to food deprivation, which present an entry point for reverse- 
engineering neural circuits for hunger. We mapped functional synaptic interactions of AGRP neurons with multiple cell populations in mice and probed the contribution of these distinct circuits to feeding behaviour using optogenetic and pharmacogenetic techniques. We have also developed tools for detailed structural analysis of AGRP neuronal connections using serial-section electron microscopy. Our results characterized some basic features of functional and anatomical circuit organization for AGRP axon projections.

Friday, 1 May 2015

14:00 - 16:00

\section{SEYMPOSIUM 3}

Experimental epilepsy

Chairs: Prof. Dr. Cafer Marangoz \& Prof. Dr. Mehmet Kaya

\section{C-012}

\section{Some features of penicillin-induced epilepsy}

Marangoz C

Department of Physiology, Ondokuz Mayıs University, Samsun, Turkey

Epilepsy is an important neurological disease from which more than 50 million persons worldwide suffer, and about $20-30 \%$ of those afflicted have seizures that are resistant to treatment with the currently available antiepileptic drugs. Studies on the epilepsy have been done in experimental models. Development of new and effective antiepileptic drugs, and the studying mechanisms of epileptogenesis require appropriate experimental models. The penicillin-induced seizure model is one of the widely studied models of experimental epilepsy. Epileptiform activity induced by certain doses of penicillin administered to mice, cats, dogs and monkeys was demonstrated in 1945. This model has many advantages as well as some limitations. The main advantages of the model can be summarized as follows: cheap and easy to apply, no significant cytotoxic effect when applied cortically, non-resistant against anticonvulsant drugs, effects completely disappear within 24 hours after penicillin injection. Penicillin induces epileptiform activity mainly through GABA antagonism. It creates ictal as well as interictal activity. The spike-wave activity induced with high dose of intramuscular penicillin in cats is similar to the activity recorded in clinical petit mal epilepsy $(3 \mathrm{~Hz}$, spike-wave activity). High concentration of potassium salt of the penicillin applied into the cerebral cortex may induce spreading depression. This is the main limitation of the model. As a conclusion, the penicillin-induced epilepsy is a valuable model of simple partial (acute), generalized myoclonic, generalized tonicclonic, and generalized absence epilepsies.

Keywords: experimental epilepsy, penicillin, mechanism

\section{C-013}

Blood-brain barrier features in kainic acid induced temporal lobe epilepsy animal models

Kaya $M$

Department of Physiology, Istanbul University Istanbul Faculty of Medicine, Istanbul, Turkey
Temporal lobe epilepsy (TLE), which is characterized by partial seizures of temporal lobe origin and resistance to antiepileptic treatment, occurs in adults following status epilepticus, traumatic brain injury, brain infection, stroke or febrile seizures. Recent studies point to blood-brain barrier (BBB) dysfunction in the development of epileptic seizures and epileptogenesis is associated with the loss of control by endothelial cells over the passage of substances across BBB. In kainic acid (KA) induced animal models, focal metabolic and electrical alterations develop in limbic structures within several hours between the injection of the acid and the resultant BBB disruption. Animal models of TLE suggest that in the course of brain injury, functional alterations in neurovascular unit components such as pericytes, astrocytes and barrier type endothelial cells take place in the more susceptible brain regions, particularly hippocampus and piriform cortex. BBB constituted by brain capillary endothelial cells is severely affected in TLE, and plays an important role in the pathophysiology of epileptic brain injury following KA intoxication. This lecture focuses on the importance of $\mathrm{BBB}$ in the pathophysiology of TLE induced by KA administration. In both acute and chronic phases of TLE, frequent vesicles containing electrondense reaction products of horseradish peroxidase tracer were present in the cytoplasm of brain capillary endothelial cells of animals treated with KA. In conclusion, the disruption of BBB integrity of animals in the course of TLE may play a major role in the pathogenesis of TLE particularly by facilitating the passage of blood-borne toxic substances into the brain.

Keywords: temporal lobe epilepsy, animal models, blood-brain barrier

\section{C-014}

\section{Interaction between cannabinoid CB1 receptor and ghrelin in penicillin-induced epileptiform activity}

Ağar E

Department of Physiology, Ondokuz Mayıs University, Faculty of Medicine, Samsun, Turkey

Experimental models have been used to obtain data about molecular mechanisms, electrophysiological basics and other properties of epilepsy. Evidences from experimental and clinical studies revealed that the CNS uses a lot of outstanding auto-protective or repair mechanisms that intend to avoid or minimize neuronal damage after epileptic seizures, such as neuropeptides. The majority of experimental and clinical studies show that ghrelin and cannabinoids are potent inhibitors of epileptic activity in various models of epilepsy. Previous studies demonstrated the connection between ghrelin and cannabinoid signalling in the regulation of food intake. Since there is no data about functional interaction between ghrelin and cannabinoids in epilepsy, we examined the relationship between these systems in penicillininduced epileptiform activity in rats. Doses of the CB1 receptor agonist ACEA (2.5 and $7.5 \mu \mathrm{g})$, the CB1 receptor antagonist AM$251(0.25$ and $0.5 \mathrm{Ig})$ and ghrelin $(0.5$ and $1 \mu \mathrm{g})$ were administered intracerebroventricularly (i.c.v.) 30 minutes after the intracortical (i.c.) application of penicillin. The administration of a $0.25 \mu \mathrm{g}$ dose of AM-251 increased the frequency of penicillin- 
induced epileptiform activity by producing status epilepticus-like activity. A $7.5 \mu \mathrm{g}$ dose of ACEA decreased the frequency of epileptiform activity. Effective doses of AM-251 (0.25, $0.5 \mu \mathrm{g})$ reversed the ghrelin's anticonvulsant activity. The application of non-effective doses of ACEA $(2.5 \mu \mathrm{g})$ together with ghrelin $(0.5$ $\mu \mathrm{g})$ caused anticonvulsant activity, which was reversed by the administration of AM-251 (0.25 $\mu \mathrm{g})$. The electrophysiological evidence from this study suggests a possible interaction between ghrelin and cannabinoid CB1 receptors in the experimental model of epilepsy.

Keywords: epilepsy, CB1, cannabinoid, ghrelin, rat

\section{C-015}

\section{The effects of melatonin in experimental epilepsy}

Yildirım M

Department of Physiology, Karadeniz Technical University Faculty of Medicine, Trabzon, Turkey

Melatonin is a neurohormone synthesized and released primarily by the pineal gland during periods of darkness. It is a component of the circadian clock that plays a significant role in the regulation of many physiological and pathophysiological functions in central nervous system, including sleep-wake cycles, mood, neuroprotection and scavenging of free radicals. Melatonin can also modulate the electrical activity of the neurons through its own receptors and other membrane receptors. Animal models play an important role in the investigation of new-generation antiepileptic drugs, and in the understanding of mechanism's underlying epileptogenesis. The relationship between melatonin and epilepsy has been studied using a variety of experimental models. Many studies have shown that melatonin has a protective effect against seizures induced by chemical or electrical stimuli such as pentylenetetrazole, pilocarpine and amygdala kindling. However, there is evidence that melatonin can have a proconvulsant effect in some animal models. In studies conducted in our laboratory, we have observed that melatonin (40 and $80 \mathrm{ug} / \mathrm{rat}$, icv) increased the latency of the first spike and reduced spike frequency of penicillin-induced epileptiform activity in rats. In another study, we also found that pinealectomy reduced the latency of the first spike and increased the spike frequency. However, melatonin $(20-80 \mathrm{mg} / \mathrm{kg}$, ip) did not provide a protection against pentylenetetrazole-induced kindling in rats. Consistent with the literature, our results indicate that endogenous melatonin signaling to have a tonic inhibitory effect on neuronal excitability and epileptiform activity.

\section{C-016}

Translation from animal to humans in temporal lobe epilepsy: a clinical and eeg findings comparison

Gürses C

Istanbul Faculty of Medicine, Istanbul University, Istanbul, Turkey

Epilepsy affects approximately 50 million people worldwide, which is about $1 \%$ of the population. Epilepsy involves econom- ic and social burden not only for the patient but also for the family. While $70 \%$ of the patients are treated with drug therapy, $30 \%$ are intractable. Among the major causes of epilepsy are genetic mutations, head traumas, brain tumors, focal cortical dysplasias. Frequent seizures, other comorbid diseases, postictal complications, adverse effects of the drugs, and sudden unexpected death in epilepsy patients (SUDEP) are the most important problems associated with epilepsy. An effective treatment targets not only epileptogenesis but also comorbid diseases. About 60 to $70 \%$ of focal seizures originate from the temporal lobe. Temporal lobe epilepsy (TLE), with its interesting symptomatology, is the most commonly researched type of epilepsy. Febrile seizure in childhood and family history of epilepsy are commonly seen in epilepsy patients with TLE. Mesial TLE is the most common type of temporal lobe epilepsies. Diagnosis is made based on clinical history, magnetic resonance imaging (MRI) and electroencephalography (EEG). Cranial MRI reveals hippocampal sclerosis in $70-80 \%$ of the patients. There are animal models for acute and chronic epilepsies. In acute animal models, seizures are induced rather than spontaneous. The effects of these studies on treatment are limited. Determining the development of epileptogenesis is necessary for the advancement of antiepileptogenic drugs. However, the impact of chronic epilepsy animal models on the development of treatment has been limited. MRI, serum biomarkers, EEG and behavioral tests on animals are good basis for translation to human patients. In order to provide the basis for translation to human, methodology needs yet to be revised and better approaches need to be developed as soon as possible. I will be discussing seizure findings on video EEG monitoring in animal models and humans with a comparative perspective.

Keywords: temporal lobe epilepsy, animal models, video EEG monitoring, epileptogenesis, comorbidity

Friday, 1 May 2015

14:00 - 16:00

\section{SYMPOSIUM 4}

Proprioception - the forgotten sixth sense

Chairs: Prof. Dr. Tayfun Uzbay \& Prof. Dr. Ersin Koylu

\section{C-017}

\section{Brain and neuroplasticity}

Uzbay T

Neuropsychopharmacology Application and Research Center (NPARC), Üsküdar University, Istanbul, Turkey

Neuroplasticity can briefly be defined as adaptive changes against internal and external stimuli in the brain's neurons, and structural and functional changes in synapses formed by these neurons. If the changes are not confined to a single neuron but reach the level of a synapse the adaptive response formed may also be called "synaptic plasticity". Long term potentiation (LTP) formation in neurons is necessary for learning and it is an adaptive response associated with neuroplasticity and synaptic plasticity. Neuroplasticity can cause positive as well as negative changes. Unfavorable neuroplasticity is characterized by an adverse adap- 
tation of the brain and appears in a number of central nervous system (CNS) disorders. Environmental factors such as stress and endogen factors such as increases in free radicals and glucocorticoids not only decrease neurogenesis but also induce apoptosis. Neurotrophic factors are always released in very low concentrations and sometimes they change neurotransmitter-mediated central neurochemical transmission. Their primary function is to help the development and regeneration of neurons, and they contribute to the important neuronal pathways for their structural health and for the maintenance of their function. Finally, neuroplasticity is a flexible re-organization, adaptation or remodeling of mammalian brain carried out by changes in synaptic formation and elements. It is affected by endogenous, exogenous and environmental stressful factors. While normal synaptic plasticity is involved in several vital functions such as mind, memory, learning, psychomotor performance and healthy behaviors, abnormal neuroplasticity may cause some serious CNS disorders such as Alzheimer and Parkinson diseases, depresyon, autism and schizophrenia.

Keywords: brain, neuroplasticity, synaptic plasticity, stress

\section{C-018}

\section{Neuroplasticity in musculoskeletal injuries}

Yertutanol Kaya FD

Neuropsychopharmacology Research and Application Center, Uskudar University, Istanbul, Turkey

Proprioception is the conscious and unconscious awareness of joint position and movement. Conscious proprioceptive pathway starts with transmission of sensory information that comes from the proprioceptive receptors of muscles and the mechanoreceptors of skin to posterior roots of spinal cord. These axons that rise in posterior column of spinal cord cross over to the other side of the medulla and project to thalamus and then somatosensory cortex. Unconscious proprioceptive pathway form spinocerebellar tract in spinal cord and project to ipsilateral cerebellar hemisphere. Coordination and regulation of motor movement is provided by integration of the proprioceptive information with other somatosensory, visual and vestibular sensory information and also feedback and feed forward mechanisms in nervous system. Due to these complex relations, the only factor that causes functional impairment in musculoskeletal injuries is not the organ itself that is injured like muscle, joint or tendon. There are some neuroplastic alterations of proprioceptive pathway that starts from receptor level in acute or chronic disorders of musculoskeletal system. First step takes place in proprioceptive receptors. Proprioception may change or deteriorate secondary to pain, trauma, effusion, immobilization and fatigue. The abnormal proprioceptive input following injury may increase need and dependence to other visual and vestibular information to manage movements. Increased nociceptive transmission in the presence of pain also contributes to neuroplastic alterations. Neuroplastic changes in central nervous system may involve spinal cord, thalamus, primary somatosensory cortex and primary motor cortex also with other cortical areas. Today, classical mechanic rehabilitation methods in musculoskeletal injuries are replaced by more comprehensive methods that involve neurocognitive interventions too.

Keywords: proprioception, pathway, neuroplasticity, musculoskeletal, rehabilitation

\section{C-019}

\section{The effects of the somatic therapatic approaches on proprioception}

Kaya D

Biruni University, Faculty of Health Sciences, Department of Physical Therapy and Rehabilitation, Istanbul, Turkey

Proprioception is the afferent information to the central nervous system provided by specialized nerve endings called mechanoreceptors that contributes to the conscious and unconscious sensation, automatic control of movement, posture, and balance. The studies from the literature have been mostly based on physiological basis of propriception, testing procedures, loss of proprioception with aging, degenerative joint disases, injuries or surgical interventions. Proprioception contributes to the motor programming for neuromuscular control required for precision movements and also contributes to muscle reflex, providing dynamic joint stability. Therefore, exercise seems crucial for rehabilitation of the proprioceptive defisits or loss of proprioception. Although proprioceptive or kinesthetic exercises are mostly included in therapeutic exercise programs for treating upper or lower extremity injuries, there is still lack of evidence based information about effect of exercise on proprioception. This presentation aims to explain possible effects of exercise on proprioception and to discuss the literature results related with proprioception using various type of exercises. Additionally, this presentation describes in details the effect of different muscle contraction types like isotonic, isometric, isokinetic on joint position sense and kinaesthetic sense. It also describes relationship between proprioception and the most common exercises such as strengthening exercises or resistive exercises, stretching exercises, close kinetic chain exercises, proprioceptive exercises, pliometrics and Tai Chi.

Keywords: proprioception, joint position sense, kinaesthetic sense, motor control, exercise

\section{C-020}

Does surgical methods affect proprioception?

Doral MN

Department of Orthopedics and Traumatology, Hacettepe University, Ankara, Turkey

Proprioception can be defined as the ability of conduction of the sense of body position, perseption and interpretation of this information, the reaction to the stimulus to move and to provide feedback information. Proprioceptors are located within the skin, muscles, tendons, ligaments and joints. İn many musculoskeletal injuries, proprioceptive feedback mechanisms are inhibited or the ability is altered. These circumstances delay the time of tissue regeneration as well as the risk of re-injury post-rehabilitation. 
For these reasons, balance education, kinetic chain excercise and sport-specific excersise should be a part of the rehabilitation in all muscluloskeletal injury with proprioceptive damage. Based on this, it is very important to address and map the proprioceptive tissues and proprioceptors within the body in order to successfully assess the medical treatment and surgery options. The care and protection of tissues rich in mechanoseptors during minimal invasive approaches, endoscopic and artroscopic operations will enhance the rate of success after surgery as well as the rehabilitation of the patient. The injury of anterior cruciate ligament (ACL) results in mechanical and functional instability and the aim of ACL reconstruction is to achieve the stabilization of the joint. Loss of proprioception following ACL rupture is due to both loss of input from the nerve ends of the ligament, and the changes in forces and kinematic motion within the joint capsule. it has been shown that major proprioceptive loss occur after ACL repair and this loss continues after reconstrutive surgical repair. It has also been shown that Ruffini, Pacini and Golgi tendon mechanosensors decline with age (Aydoğ ST, Korkusuz P, Doral MN, Tetik $\mathrm{O}$, Demirel HA. Decrease in the numbers of mechanoreceptors in rabbit ACL: the effects of aging. Knee Surg Sports Traumatol Arthrosc. 2006). It has long been recognized by surgeons that the loss of sense related to ligamentous damage can affect the results of surgical repair and replacement. Theoretically, surgical techniques remediate proprioception directly through the reinnervation of damaged tissue or indirectly through reduction of strain within capsuloligamentous tissue to its normal value. It should be noted that unnecessary surgery without the set of proper indication enhances the risk of complication and alters the deep sensory return. Proprioceptive excercise is effective in the construction of this way, and the importance of brain function should not be underestimated. The questions that need to asked can be listed as below: 1) Can the receptors be produce in the laboratory? 2) When a tissue with the receptors is removed from the body, which other receptors are taking their place, is there any increase in their numbers, and how can these be measured if so? 3) Is there any ratio between the number of functional muscle fibers and receptors?

Friday, 1 May 2015

$17: 45-18: 30$

CONFERENCE 5

Functional architecture of spatial circuits in the brain Chair: Prof. Dr. Erdal Ağar

\section{C-021}

\section{Functional architecture of spatial circuits in the brain}

\section{Witter MP}

Norwegian University of Science and Technology, Trondheim, Norway

The representation of space and self-location is mediated by a diversity of neural circuits involving the hippocampal region, an area of the brain that is the core component of our conscious memory system. The neural circuits involved comprise a number of functionally specialized cell types, which among others code for location, directionality and velocity. Historically, the system appeared neatly organized, with individual functional cell-types belonging to unique neuronal networks, organized in a serial fashion. The current view is that the system is a complex parallel system. In my presentation, I will describe the functional cell types found in this brain region and give an overview of the wiring of networks that may underlie this functional diversity.

Saturday, 2 May 2015

08:30 - 10:00

PANEL 1

Different approaches to etiopathology of childhood

psychiatric disorders

Chairs: Doç. Dr. Sabri Hergüner \& Doç. Dr. Yavuz Selvi

\section{C-022}

Do androgens play a role in the etiology of neurodevelopmental disorders?

Hergüner $\mathrm{S}$

Department of Child and Adolescent Psychiatry, Necmettin Erbakan University, Meram Faculty of Medicine, Konya, Turkey

Childhood neurodevelopmental disorders (e.g. autism, attention deficit hyperactivity disorder, tic disorder) more frequently observed in male gender. Several studies suggested that intra uterin fetal testesteron levels might be responsible for that gender prepondarence. Indirect measurement of intra uterin testesteron levels is ratio of the second digit to fourth digit. Both in autism and attention deficit hyperactivity disorder 2:4 ratio was found to be more masculin than normal developing subjects. The association between fetal androgens and neurodevelopmental disorders is also tested in several disorders (e.g. congental adrenal hyperplasia, polycystic ovary syndrome) which were associated with excessive androgen levels. Subjects with CAH and PCOs were shown to have higher autistic traits and hyperactivity levels. Our group also showed a close relation between age at menarche and autistic traits in non clinical university female population.

Keywords: androgens, male gender, autism, children

\section{C-023}

Psychiatric disorders and vitamin D

Gurkan K

Department of Child and Adolescent Psychiatry, Ankara University, Ankara, Turkey

In this presentatiton the role of vitamin $\mathrm{D}$ in psychiatric disorders will be discussed. Vitamin D has important functions in bone, brain and whole body. It is thought that vitamin D has a role in regulation of cellular growth, adhesion, DNA restructuring, apoptozis, membrane transport, oxidative stres and many other bodily functions. It also has importtant function in gene regulation. In light of the recent literature it is thought that vitamnin D deficiency may play a role in pathogenesis of some psychiatric disorders including autism, schizophrenia and depression. I try to explain the role of vitamin D in psychiatric disorders.

Keywords: autism, schizophrenia, depression, gene regulation, vitamin D 


\section{C-024}

Neurotrophins in childhood onset psychiatric disorders

Bilgiç A

Department of Child and Adolescent Psychiatry, Necmettin Erbakan University Faculty of Medicine, Konya, Turkey

Neurotrophins have been considered as key mediators for the development and maintenance of neurons, neuroplasticity, neuronal connectivity, and the regulation of the activity of many neurotransmitter systems. Although data is not universal, neurotrophins have been reported to be related to various psychiatric disorders such as depression, bipolar disorders, schizophrenia, autism spectrum disorders, and attention-deficit hyperactivity disorder. Furthermore, it has been suggested that an alteration of neurotrophin concentrations in some patients might reflect abnormal prenatal and early postnatal neural development. This debate has aimed to review the possible impacts of neurotrophins for the development of childhood onset psychiatric disorders.

Keywords: neurotrophins, psychiatric disorders, child

\section{C-025}

\section{Anxiety and relationship with visual perception}

\section{Yilmaz S}

Department of Child and Adolescent Psychiatry, Necmettin Erbakan University, Meram School of Medicine, Konya, Turkey

Visual perception is a complex process that can be affected by a variety of factors, such as emotion, motivation, life experiences, condition, and the location of the person or perceived object. Most studies investigating the relationships among anxiety, fear, and visual perception have found that anxious individuals demonstrate cognitive biases, especially with regard to threat-relevant stimuli. Among individuals with specific phobias, a small snake may cause an exaggerated behavioural reaction, a harmless spider may appear to move at breakneck speed, and a neutral facial expression can suddenly appear to be a threatening stimulus. For example, a spider depicted in a video as crawling toward them is seen as faster and larger by individuals who fear spiders than by non-fearful individuals. Likewise, fearful individuals verbally report higher estimates of the ?ickering tongue movements of a snake and of the jumping movements of a spider compared with non-fearful individuals; these biases diminish following fear reduction. Similarly, subjects more frequently overestimate the height of a balcony after imagining themselves falling, particularly if they were already afraid of heights. Height is consistently overestimated when subjects are asked to estimate the distance from the top rather than from the bottom of a balcony Stefanucci and Proffitt, and this overestimation is thought to be due to fear. Previous studies have demonstrated that perceptual distortions occur under anxiety-provoking conditions, but data regarding the relationship between anxiety and the perceived size of a neutral object remain limited. Visual perception and anxiety relationship may be bidirectional. In this presentation, this relationship discuss with recent findings.

Keywords: visual perception, anxiety
Saturday, 2 May 2015

08:30 - 10:00

PANEL 2

Psychiatric disorders and inflammation

Chairs: Prof. Dr. Gönül Peker \& Prof. Dr. Dilek Taşkıran

\section{C-026}

\section{A time tunnel yet to be enlightened:} brain-mind-body-behavior

Peker G

Department of Physiology, Ege University, Faculty of Medicine, Izmir, Turkey

The problematics of the unity or duality of the brain and body, and whether a collaboration or coordination exists between them have been major subjects of philosophy, theology and science for millennia. Thales, Pythagoras, Plato, Socrates, Anaxogoras, Hippocrates, Alcmaeon of ancient Greece have marked the Golden Age of Philosophy by their teachings of various scopes. Orthodox Christian dogma (including exorcism to heal the psychiatric patients) have domineered the Medieval Age despite the efforts of a few scholars striving to address the issues integrating theology and philosophy. However, corruption of the Church and the so called Holy War (Crucaders) were inevitable. St. Augustin revisited Plato while the Islamic world identified with positivism. Avicenna revived Aristoteles and studied intellect through senses as a whole. Nevertheless, he stated the importance of the seven sub faculties or components of the integrated intellect in the following rank order: Practical intellect, processing information, memory, cognition, creative imagination, the five physical senses, and utilization knowledge gained. Eventually, intellects crossed the intrepid waters to split reason from faith and religious doctrine. Renaissance triggered the scientific revolution, and empiricism became stronger. René Descartes tried to explain the interaction (Interactionism) of the physical with non-physical in terms of dualism, stated pineal as the home of the spirit and someway superposed the spirit (mind) with the brain and nervous system. Spinoza seemed to confirm at the beginning but later proposed his own understanding of dualism. Following Descartes, empiricist John Locke argued that each human start as tabula rasa and the essence is what he/she acquires through life. Against the Cartesian Dualism, Leibniz stated the Doctrine of Parallelism as the second type of dualism. In the 17 th century, Malebranche, defined Occasionalism, briefly accepting dualism however, refusing interactionism. Thomas Hobbes, a strong advocate of Monoism, declared that all cognitive processes are physical matters and can be thoroughly explained by materialistic approach. In the Age of Enlightenment (18th and 19th centuries), the understanding of brain science and the interdependence of the mind and body led the way to new thoughts and oriented the "mind-body dichotomy". Taught by the positivist (scientist) Robert Boyle, his scholar Locke opened and developed experimental and theoretical avenues to understanding of human fear, education, government, and behavioral therapy. Another reflection to past characterized by empiric and spiritual approaches was made by George Berkeley who said that "the essence is not the matter but it is definitely the idea". David Hume, originally a real supporter of Berkeley, defined a very strong distinction between 
impressions (strong perceptions) and ideas (weak perceptions). In the 19th century, popular German master of letters and Nobel Laureate, Thomas Mann, inspired by an archaic Indian mythology published a short novel entitled, "The Transposed Heads" which although lavish and melodramatic, functioned as a real breakthrough for the intellects as well as the laymen and led to the modern understanding the brain-body interaction and the sound interdependence between them. This set the setting for foundation of psycho-neuro-endocrinology. Meantime, the real tragic case of the American railroad worker Phineas Gage who survived a severe blast injury, but later developed mental and behavioral disorder set the retrospective experimental and materialistic background for today's understanding of inter-dependence among brain, mind and behavior. Starting from 540 BC, Heraclitus, Empedocles and Hippocrates, then the 19th century scientist, Claude Bernard, and finally the 20th century physiologist Walter B. Cannon coined the terms and the underlying evidence-based bio-facts such as "milieu intérieur", "homeostasis", and more recently, "allostasis" and "homeodynamics" one after the other. In the first half of the 20th century, "Translational Decades" came by relying mostly on the data from Hans Selyé's ground breaking studies and resultant statements of "stress, stressors, and stress responses" also leading the way to the understanding of "psycho-neuro-endocrino-immune" biology and medicine. Then at least two generations of bright students from Selyé's lab demonstrated that "the brain is not a prohibited site or a conservatory" for immune responses and factors (cytokins) originating in the body or periphery. These scientists also showed that both CNS- and PNS-specific glia were actors of much more complex roles. More recently, neuroscientists proved that glia can play either the friends or foes in protection and restoration of the CNS and PNS in a context-dependent manner. Also, these responses and interactions are not exclusively valid in neurologic disorders but are capable of making both the brain and the body sick at the same time in psychiatric disorders.

Keywords: monoism, dualism, positivism, materialism, spiritualism

\section{C-027}

\section{Inflammation and sickness behavior}

\section{Taşkıran D}

Department of Physiology, Ege University School of Medicine, Izmir, Turkey

Inflammation is a protective response of immune system to pathogen and/or tissue injury. Physiologically, the purpose of inflammation is to remove the pathogen and necrotic cells and to repair tissue damage. Inflammatory response involves immune cells, blood vessels, and molecular mediators such as cytokines, acute phase reactant proteins, chemokines, and adhesion molecules. Several studies indicate that pro-inflammatory cytokines, produced by monocytes, macrophages and brain microglia, play an essential role as mediators of external and internal stress responses. The pro-inflammatory cytokines including interleukin-1 (IL-1), interleukin-6 (IL-6), and tumor necrosis factor alpha (TNF- $\alpha$ ) lead to a breakdown of the blood-brain barrier and influence the synaptic neurotransmission in the brain. Also, these cytokines may increase the central expression of NF- $\mathrm{NB}$ that triggers transcription and secretion of pro-inflammatory cytokines in the brain. Both in animals and humans, systemic inflammatory pathways can induce "sickness behavior", including behaviors such as fatigue, anhedonia, anxiety, weight loss, impaired concentration and sleep disturbances. Several plasma inflammatory biomarkers have been found to be increased in patients with major depressive disorder. Furthermore, clinically, there is a strong correlation between major depressive disorder and chronic inflammatory disorders, such as diabetes mellitus, metabolic syndrome, and obesity. The chronic administration of antidepressants systemic lessens inflammatory response and stimulates synaptic plasticity, axonal growth and regeneration. Although the precise mechanism of antidepressants is unclear, recent studies suggest that they can suppress the release of proinflammatory cytokines and other immunological parameters.

Keywords: inflammation, sickness behavior, depression, cytokines

\section{C-028 \\ Cytokin related behavioral changes and inflammation in psychiatric illnesses}

Erbaş O

Department of Physiology, Bilim University, Istanbul, Turkey

A mild degree of peripheral inflammation in psychotic patients has been detected and reported in the literature. Investigators indicate that bipolar patients and schizophrenics express elevated levels of IL-6, and serum oxidative stres and TNF-alpha, respectively. Stress, obesity, genetic factors and nutritional styles induce inflammation by upregulated release of cytokins such as IL-1, IL2, IL-6, and TNF-alpha which may be of peripheral origin and capable of crossing the blood-brain barrier and / or come into play via activation of the brain glia. In the central nervous system, the cytokins modulate dopaminergic, glutamatergic and GABAergic action, thus yielding to "sickness behavior" comprising of a series of disrupted conditions and symptoms. Additionally, psychotic patiens have been reported to express rather TLR-4 (Tolllike receptor 4) exclusively, for which these patients' disturbing mucosal inflammation had been attributed to this subtype. Psychiatric illnesses are found to co-exist with irregular neurotransmitter release which inflammation is most often blamed for. Advancement in this area of research is likely to improve / optimize novel therapeutic agents and more efficient strategies.

Keywords: sickness behaviour, inflammation

\section{C-029}

\section{Inflammation in neurodegenerative processes}

\section{Solmaz V}

Turhal State Hospital, Tokat, Turkey

The site of neuro-inflammatory onset can be either the center or periphery of the nervous system. In neurodegerative processes, inflammation emerges at the very beginning or at some point of time during the ongoing course. The order of causation - in 
other words - neither the cause nor the effect can distinctly differ from one another. Most certain fact is that there is no neurodegeneration without inflammation. The highest incidence neuro-clinical cases such as Alzheimer's, Parkinson's, neuropathic and demyelinating disorders, and also various subtypes of epilepsy benefit from anti-inflammatory medication almost inclusively. There definitely remains a lot more to unveil, define and identify the multifaceted nature of neuro-inflammation so that more effective and target-specific strategies can be developed. Only then, it may be possible or combat or supress the inflammation which may either emerge to set the stage for igniting the cascade of destructive events or worsening the resultant burden of neuro-degeneration.

\section{C-030}

Essential pathophysiological mechanisms underlying psychiatric disorders and their relation to inflammation Akseki HS

Nazilli State Hospital, Nazilli, Aydın, Turkey

Recent research has shown that there may most probably exist strong interrelations between psychosis and immunity / inflammation. Several publications have clearly reported evidence for inflammation at the time of the very first episode in patients who had not been on any anti-spsychotic treatments. These patients of mention expressed significant cytokin-positivity for various cytokins. We have also performed a series of rat models for severe metabolic disfunction, and obtained significant data supporting the relation between simulated sickness and psychotic behavior. Other investigators demonstrated that several factors activating infection and microglial upregulation have affected neurogenesis, thus inducing significant predisposition and vulnerability to psychosis. The endophenotype phenomenen emerging as an intermediate feature during the long course from genes to clinical cases are detectable with sensitive techniques in the present day. This data obtained may serve as an important key in advancing our studies and understanding of psychosis in clinical cases and experimental paradigmas. Collectively, unveiling the interrelation between either psychosis or other psychiatric disorders and inflammation seems definitely promising to enlighten the pathophysiology of such disorders and help develop novel strategies to combat the affected humans.

Saturday, 2 May 2015

10:30 - 11:15

\section{CONFERENCE 6}

Burden of brain disorders: a global perspective on the impact on patients, families, societies and policies

Chair: Prof. Dr. Gürkan Öztürk

\section{C-031}

Burden of brain disorders: a global perspective on the impact on patients, families, societies and policies

\section{Leonardi $M$}

Department of the Neurological Institute, Carlo Besta IRCCS Foundation Milan, Italy
Brain disorders represent a great challenge for the economy of several countries of the world. They have in fact large costs for the individuals as well as for societies mainly due to epidemiological transition and to compression of morbidity, due to ageing. Neurological and mental disorders compose a significant proportion of the global burden of disease. The WHO and World Bank Global Burden of Disease study, last update GBD2010 published in 2012, measures burden with four summary measures of health that address disability associated to diseases across countries. In GBD2010 mental and behavioural disorders comprised $7.4 \%$ and neurological disorders $3 \%$ of the global burden of diseases. However these categories don't include meningitis, encephalitis, HIV/AIDS, chronic pain, head and spinal cord injury and stroke that alone accounts for an additional $4.1 \%$ of the total GBD. Inclusion of these categories greatly increases the estimate of brain burden. Although GBD measures of health aren't directly translatable into monetary cost the European Brain Council, EBC, has brought to the attention of the international community the costs associated to the high burden of brain disorders since 2003. EBC filled the knowledge gap related to costs by providing sound estimates of costs of brain disorders in Europe with two subsequent EBC cost studies. The first, in 2004, was prevalence based and pointed out that 127 millions of Europeans lived with a brain disorder for a total cost of 385 billion euro (62\% due to psychiatric disorders and $38 \%$ due to neurological disorders). The EBC 2010 cost study updated the costs of brain disorders in 30 European countries and showed that one third of all European citizens had at least one brain disorder accounting for 798 billion euro per year that include direct medical (37\%) and non medical costs (23\%) and indirect costs (40\%). It is clear that brain diseases are the current and future major health economic challenge for many countries. To face this societal emergency a strong networking of basic and clinical research, of public health policies and of involvement of relevant stakeholders is needed.

Keywords: burden, brain disorders, costs, disability, research

Saturday, 2 May 2015

10:30 - 11:15

\section{KONFERENCE 7}

Molecular anatomy of cortical projection neurons

Chairs: Prof. Dr. Esat Adıgüzel \& Prof. Dr. Fatma Sultan Kılıç

\section{C-032}

Molecular anatomy of cortical projection neurons

Ulupinar E

Department of Anatomy, Eskisehir Osmangazi University, Faculty of Medicine, Eskisehir, Turkey; Interdisciplinary Neuroscience Department of Health Science Institute, Eskisehir Osmangazi University, Eskisehir, Turkey

Cortex is not only very complex, but also a highly organized structure, containing hundreds of different neuronal cell types. Within this region of the nervous system, two broad classes of cortical neurons; interneurons and projection neurons make local or distant connections, respectively. Neocortical projection neu- 
rons having unique morphological features are generally located in different cortical layers, express different transcription factors and work for different functions. However, relatively little is known about the molecules and mechanisms that control the commitment of progenitors giving rise to diverse groups of projection neurons. In recent years, remarkable advances have been made in identification of laminar- and subtype-specific markers by using current molecular techniques and comparing the expressions of genes in purified neuronal populations. In addition, lossof-function experiments in null mutant mice have been demonstrated their functional significances. In this talk, I will first review the development of the rodent neocortex in the context of current data regarding the role of individual genes in controlling the specification of distinct projection neuron subtypes. Then, I will focus on subcerebral projection neurons as a clinically important neuronal population; since they consist of corticospinal motor neurons that degenerate in motor neuron diseases. And finally, I will present our experimental findings from a novel animal model that displays profound cell loss in the corticospinal motor neurons via increased endoplasmic reticulum stress.

Keywords: neocortex, projection neurons, animal models

Saturday, 2 May 2015

14:00 - 15:45

PANEL 3

Visual states without an object - dream, imagination, hallucination Chairs: Prof. Dr. Mustafa Sercan \& Prof. Dr. Lütfü Hanoğlu

\section{C-033}

Visual hallucinations in terms of neuropsychiatric and environmental factors

Sercan M

Turkish Neuropsychiatric Society, Istanbul, Turkey

Hallucinations are abnormal psychic experiences. Voluntary (meditation) or involuntary (illness, punishment, torture) solitude, sleep deprivations and chemical stimulants are hallucinogen. Sleep deprivation may be a trigger or outcome of some psychiatric symptoms and cortical excitability that provokes hallucination and seizure (Babkoff $\mathrm{H}$ et al., 1989) correlates negatively with duration of being awake (Huber R et al., 2013). Stimulants (LSD, cocaine) may cause hallucinations too by neurotransmitter pathways and fluctuations on neurotransmitters (dopamine, serotonin, asetylcholin, glutamate, NMDA) involve hallucination. In the aspect of neurobiology, schizophrenia disrupts the sequential proceeding of stimulations (Braff et al., 1995). Failure in filtration of sensory incomes is relevant abnormality of perception and attention in schizophrenia (Venables 1964). Schizophrenia accompanies a different default mode network (DMN) that is organized by anterior and posterior cingulate cortex (Garrity et al., 2007). Temporal fluctuations in regulation of DMN may be concluded changes in connections intra and inter areas of brain. In an fMRI research, task-related suppression of activation in DMN of schizophrenia patients and relatives have markedly reduced (Whitfield-Gabrieli et al., 2009). The DMN that is responsible for periods of autobiographical 'task independent' (Ballard et al., 2011; Mazoyer et al., 2001), the Dorsal Attention Network (DAN) that encodes behavioral significance of salient stimuli, particularly in the visual domain (Asplund et al., 2010; Kincade et al., 2005) and the Ventral Attention Network (VAN) that coordinates activity between DMN and DAN, provides together correct perception by interactivity (Corbetta and Shulman, 2002). Disorders in different parts of this system appear visual misperception. 'Simple' visual hallucinations arise from disturbances within regions responsible for the primary processing of visual information; in contrast, 'complex' visual hallucinations reflect dysfunction within and between the Attentional Control Networks (especially DAN) (Shine et al., 2014). As a result, isolation of brain from external stimulation, certain personality structures, disorders or lifestyles, rising in excitability of brain are considerable as facilitator the hallucinations. In the view of neurobiology; neurotransmitters, temporary or permanent imbalances in some activity of sensory cortex or activity of networks or structures for organizing attention may explain the processing of visual hallucination. It is required to concentrate on using in treatment.

\section{C-034}

\section{Seeing without an object: dreaming}

Yalçıner B

Neurology Clinic, Içerenköy Bayındır Hospital, Istanbul, Turkey

As a result of brain-scanning studies that have been going on for 30 years, it has been found that there is an anatomically defined specific brain system that is activated especially when a subject is not focused on exterior stimuli. These findings have started to be presented in meta-analyses of fMR studies: Resting state networks - "Default Mode Network (DMN)" -have been identified as a new brain system that plays a role in the formation of cognition, and its importance has only recently been recognized. $\mathrm{DMN}$ is a brain system, just like the motor system or the visual system. This network system, which consists of a number of inter-related sub-systems, contains interactive brain areas that have strong functional relationships to the other systems of the brain. While it is known that DMN activity is maintained during light sleep, recent studies have shown that it is also maintained in deep sleep; however, the functional connection of MPFC with the remaining DMN segments is broken. This finding will enable us to make further evaluations regarding misperceptions during Lucid dreaming and sleep.

\section{C-035}

\section{Epilepsy and hallucinations}

\section{Özkara Ç.}

Department of Neurology, Istanbul University, Cerrahpasa Faculty of Medicine, Istanbul, Turkey

Seizure is the transient findings caused by excessive, hypersynchronous discharges of a group of neurons in the brain and epilepsy is the chronic condition in which seizures occur without 
any provoking factor. Phenomenology in epileptic seizure is related to the anatomic structures and pathways effected by spreading discharges of the seizure. The patient can be unaware in some seizures, while in others can remember the whole incident. Seizure in which subjective sensations can be perceived by the patient but not by a second party is called aura. In particular there are sensory, emotional, visual, gustatory and olfactory or auditory auras and the patient personally can give us this information. Because patients can communicate during nondominant hemisphere seizures, sometimes the sensations can be described in detail. Especially when the seizure involves the regions of visual, auditory or olfactory functions, visual, auditory and oflactory hallucinations occur by irritative effect. While elementary images like bright lights or shapes are perceived in primary cortex involvement; more complicated, structured images like people or events are perceived in the involvement of association cortexes. Similarly superior temporal cortex related seizures generate simple auditory hallucinations, while secondary auditory cortex involvement can create a perception of change of surrounding sound. Eventhough the pathophysiology is not yet fully clarified, epileptic discharges are thought to be noise causing disruption within the hierarchical system of central nervous system. It is speculated that the discharges away from visual network but within association areas are perceived as a new object or the actual image may be disrupted or changed. Especially as rhinal cortical structures are localised between functional ventral streams and hypocampal streams an abnormal synchronisation can lead to a interpretation error. This error is in the form of signalisation rather than coding of signals and it is hypothesised as "coding experienced in the form of recall". In this way, déjà vu or events that occur in the form of paroxysmal recollection may be associated with increased synchronization between hippocampus and cortex. It is known that the network which provides recollection is wide and extends to association areas. Some conditions are accompanied by function loss while in some conditions increase of the function can be seen. On the other hand depending on the impressions obtained from the epilepsy patients, it is thought that especially the more complicated hallucinations may be due to the liberalization of traumatising events in the implicit memory in the form of seizure. This issue will be discussed with examples of seizures.

\section{C-036}

Parkinsonian visual hallucinations and their mechanisms

\section{Hanoğlu L}

Department of Neurology, Istanbul Medipol University, Istanbul, Turkey

"Creating maps is one of the distinctive elements of the brain." says Damasio. This action of the brain causes the images to come existence, which are the principle elements of the brain. Building of maps/creating images on our mind is a continuous process; even when asleep the maps are created as we dream. These maps in our minds contain the information about an outer object, movements, the relationship of this object with the others, the relationship between the brain and its body and they recreates this information within the time and space dimensions in our mind. This maps are not simple copies of outer objects. Whatever the source, these inner images have, they are always principally the same. Always the same regions of the brain are responsible for the creation of these images. How can we be sure about the reality of the visions, thoughts, meanings, fictions; which occupy our mind. How can we be sure about our "mind concepts", which we found ready and naturally in our mind, being a true reflection of our outer reality, not a fiction that plays a shenanigan on us, or a dream, or even a hallucination and except for these how can we know when one or the other is present? So, the visual hallucinations in patients with Parkinson's disease are a good neurological model for building the answer of these questions. In this speech it is going to be reviewed and argued the knowledge in this field through these concepts and questions.

Keywords: hallucination

Saturday, 2 May 2015

14:00 - 15:45

\section{SYMPOSIUM 5}

Pericytes: Newly discovered significance of neglected cells Chairs: Prof. Dr. Turgay Dalkara \& Prof. Dr. Alp Can

\section{C-037}

\section{Pericytes and vessel microenvironment revisited}

Can A

Department of Histology and Embryology, Ankara University School of Medicine, Ankara, Turkey

Pericytes, which make intimate connections with adjacent capillary endothelial cells are the mural cells of blood microvessels, have recently come into focus as regulators of vascular and skeletal muscle morphogenesis and innervation; function during development, cardiovascular homeostasis, fat accumulation, ectopic bone formation and some diseases such as Alzheimer disease. Pericytes are implicated in the development of diabetic retinopathy and tissue fibrosis, and they are potential stromal targets for cancer therapy. Due to their heterogenic property some pericytes are probably mesenchymal stem or progenitor cells, which give rise to adipocytes, chondrocytes, osteblasts, fibroblasts and muscle cells. However, there is still confusion about the identity, ontogeny, and progeny of pericytes. Here, in this presentation the history of these investigations, indicate emerging concepts, and point out problems and promise in the field of pericyte biology will be discussed.

Keywords: pericyte, vessel

\section{C-038}

\section{Pericytes in brain: friend or foe?}

\section{Yemişci $M$}

Institute of Neurological Sciences and Psychiatry and Department of Neurology, Faculty of Medicine, Hacettepe University, Ankara, Turkey

Pericytes were localized to microvasculature after 50 years, following their first identification in 1873. Recent studies highlighted the importance of pericytes, by their critical location around the microvessels in brain and retina, for maintenance of blood 
brain barrier function, regulation of endothelial cell functions and angiogenesis. As a continuation of vascular smooth muscle cells in large vessels, the contractile properties of pericytes make them significant modulators of blood flow at the microcirculatory level especially in pathologic conditions. Their role in modulating capillary blood flow in physiologic conditions and their role in physiologic neuro-vascular coupling has not been elucidated sufficiently. Studies have shown that capillary pericytes constrict in response to neurotransmittes and vasoactive molecules. The contractile property of pericytes adversely contributes to the pathophysiology in certain pathologic conditions like ischemia. Ischemic stroke is the most common cerebrovascular disease, and the most common causes of death, and leading cause of disability in the adult population. Currently approved therapeutic approaches in ischemic stroke primarily aim for the recanalization of occluded arteries; yet cannot establish satisfactory improvements in microcirculatory blood flow despite successfully fulfilling this aim. The impaired blood flow in the microcirculation is accompanied by insufficient delivery of critical elements for neuronal and glial survival,like glucose and oxygen. Pericytes were shown to contract during this ischemia/reperfusion in the brain and remain contracted despite restoration of blood flow in larger vessels, thereby preventing the establishment of reperfusion in the microcirculation (no-reflow phenomenon). This leads unfavorable tissue and clinical outcome following cerebral ischemia. The prevention of oxidative and nitrative damage has been demonstrated to reverse partially this pathologic contraction in pericytes. The understanding of the physiologic functions of pericytes is imperative not only for understanding the dynamics of cerebral blood flow at the microcirculatory level but also for alleviating the pathologic responses associated with them.

Keywords: brain, pericyte, ischemia

\section{C-039}

\section{When pericytes turn into the baddies}

Alarcon-Martinez L

Institute of Neurological Sciences and Psychiatry, Hacettepe University, Ankara, Turkey

Pericytes are essential cells in CNS, having important functions. However, during cerebral ischemia, pericytes constrict microvessels, which do not relax after recanalization. Since the retina contains the highest density of microvascular pericytes of any organ, retinal reperfusion after ischemia may also be impaired by pericyte-induced microvascular constrictions. Here, we analyze whether pericytes constrict retinal microvessels during in vivo ischemia and hinder reperfusion. To do it, we used wild type $(\mathrm{n}=35)$ and NG2-DsRed $(\mathrm{n}=10)$ mice. We induced clot formation in the central retinal artery by topical $20 \%-\mathrm{FeCl} 3$ application over the artery for 3 minutes. After 60 minutes of ischemia, we infused tissue plasminogen activator (tPA) through tail vein to induce recanalization. We monitored the retinal blood flow by laser speckle contrast imaging. We also imaged retinal pericytes and blood flow in vivo under conditions of normal perfusion, ischemia, and reperfusion by using a two-channel adaptive optics scanning laser ophthalmoscopy (AOSLO). After, we labeled whole mount retinas ex vivo with markers for pericytes and ves- sels and, stereologically counted the constrictions and vessel diameter. We found a significantly higher number of microvessel constrictions and a decreased microvessel diameter in ex vivo ischemic $(n=3)$ retinae compared to the sham group $(n=3)$. Constrictions were not restored after recanalization of the retinal artery $(n=3)$. We confirmed these observations with in vivo retinal imaging $(\mathrm{n}=10)$. There was a significant co-localization between pericytes and in vivo microvascular constrictions. Finally, we found a novel mechanism that explains how pericytes constrict microvessels under ischemic conditions. Thus, after ischemia, pericytes constrict microvessels permanently and lead to incomplete microcirculatory in reperfusion.

Keywords: pericyte, ischemia, reperfusion, retina, imaging

Saturday, 2 May 2015

16:15 - 17:00

CONFERENCE 8

Multiple sclerosis and neuroimmunology

Chair: Prof. Dr. Şerefnur Öztürk

\section{C-040}

\section{Multiple sclerosis and neuroimmunology}

Rieckmann P

Department of Neurology, University of Erlangen-Nürnberg, Bamberg Academic Hospital, Bamberg, Germany

Multiple sclerosis (MS) is the most common inflammatory, demyelinating, neurodegenerative disorder of the central nervous system (CNS), leading to chronic neurological defecits in young adults. It is widely considered a T-cell mediated autoimmune disease that develops in genetically susceptible individuals, possibly under the influence of certain environmental trigger factors and vitamin $\mathrm{D}$ deficiency. The invasion of autoreactive CD4+ T-cells into the CNS is thought to be a central step that initiates the disease. Several other cell types, including CD8+ T-cells, B-cells, macrophages and glial cells oft he CNS appear to be involved in causing inflammation and eventually neurodegeneration. But inflammation is not entirely deleterious in MS. Evidence has accumulated in the recent years that demonstrate the importance of regulatory immune mechanisms restraining tissue damage and initiating aregeneration. Another important aspect of neuroimmunology in MS is related to mechanisms of autoimmune cell activation, a process essential for immune cell migration across the vlood brain barrier. While activation has been traditionally associated with microbial infections, recently, studies of animal models revealed a critical role of the commensal gut flora as a key triggering factor. These findings may pave the way to new strategies to treat MS and other human autoimmune diseases, and commend a reevaluation of dietary approaches.

Saturday, 2 May 2015

16:15 - 17:00

\section{CONFERENCE 9}

The problem of self of un-conscious psychology

Chair: Prof. Dr. Serdar Gergerlioğlu 


\section{C-041}

The problem of self of un-conscious psychology

Kara $\mathrm{H}$

Istanbul Erenkoy Hospital, Istanbul, Turkey

Consciousness was considered as a subjective experience; for this reason it was not involved in the scientific research as a topic for a long time. What is more, the science of mind, psychology, had been resistant to accept the consciousness and related concepts until to the last quarter of the 20th century. It is ironic that this resistance was over after the discovery of quantum physics. Classic physics is based on the fact that there is an objective reality independent of the existence of an observer. According to the classical physics, the universe is out 'there' and functions unconsciously by its own deterministic laws. Modern psychology is based on this model of universe. However, quantum physics states that without the existence of an observer, world of subatomic particles cannot be described. Psychology, involuntarily, had to go along with this revolution of thought led by quantum physics after fifty years of resistance. This revolutionary change of the idea of consciousness made us to confront with a new problem:the problem of self. As F. Brentano said 'consciousness is always the consciousness of a thing'. This simple reality refers not only to the objects of consciousness, but also to the self who is the conscious being. The questioning of the self is a very hard theme and there is an amazing background for this hardship in the intellectual history of the western societies. As Nietszche stated, western ideology has shrewdly tried to get rid of the concept of self since Descartes;even has tried to make the self an object of mind. Actually, this historical war against the self is also reflected on the resistance of modern psychology to accept consciousness. In this presentation, concepts of self, consciousness and unconsciousness will be discussed by considering this historical perspective, especially from the stance of psychology.

Sunday, 3 May 2015

08:30 - 10:30

\section{SYMPOSIUM 6}

Networks of brain connectivity from the perspective of basic and clinical neurosciences

Chairs: Prof. Dr. Tamer Demiralp \& Prof. Dr. Metehan Çiçek

\section{C-042}

Investigation of the functional connectivity in the brain by using $\mathrm{fMRI}$

Demiralp T ${ }^{1}$, Kurt E ${ }^{2}$, Ulaşoğlu $C^{2}$, Kıçik A², Bayram A Özbek S

'Department Of Physiology, Istanbul University, Istanbul Faculty Of Medicine, Istanbul, Turkey; ${ }^{2}$ Hulusi Behçet Life Sciences Research Laboratory - Neuroscience Unit, Istanbul University, Istanbul, Turkey; ${ }^{3}$ Biomedical Equipment Technology Department, Üsküdar University, Istanbul; ${ }^{4}$ Department Of Neuroscience, Istanbul University, Institute For Experimental Medical Research, Istanbul, Turkey

Functions of the nervous system are based on connectivities in multiple spatial scales forming the microcircuits and small- and large-scale neural networks. Neural processes also occur in a wide temporal range starting with electro-chemical activities at millisecond scale running along the metabolic and hemodynamic responses at second scale to longer lasting neuro-modulation and plasticity processes. Understanding the neural basis of brain functions necessitates the integration of these multiple tempo$\mathrm{ral} /$ spatial scales. Although electrophysiological measurements capture neural activities at millisecond resolution, they are limited in displaying spatial characteristics due to biophysical reasons. First non-invasive 3D localization of function in the nervous system was possible with positron emission tomography (PET). This has been followed by the development of the hemodynamic response and tissue perfusion measurements by using magnetic resonance imaging (MRI), which due to neurovascular coupling allow imaging of neural activity. fMRI measurements that became popular for detecting brain regions activated during specific tasks, later were developed for neural network and connectivity analyses that are more relevant for understanding brain functions. Functional and effective connectivity analyses were carried out by investigating the correlations among brain areas during tasks. In last years, resting state or intrinsic connectivity networks came to foreground on the basis of fMRI correlations among brain regions during resting condition. These functional connectivity patterns are correlated with anatomic connectivity observed by other MRI based techniques. In this talk, I will shortly explain fMRI based functional connectivity analyses and present examples based on our recent work on healthy controls and ADHD patients.

Keywords: fMRI, functional connectivity, neural networks, temporo-spatial anlysis, intrinsic connectivity networks

\section{C-043}

\section{Assesment of effective connectivity of the brain with fMRI}

\section{Çiçek M}

Department of Physiology, Ankara University Faculty of Medicine, Ankara, Turkey

We know understand that to understand the brain we should understand the neural networks. We can assess the connectivity of human brain with structural and functional methods. Functional magnetic resonance imaging is one of the most frequently used functional method. Brain mechanisms during cognitive processes evokes neurovascular activity. This activity causes magnetic differences and can be recorded by fMRI. Functional and effective connectivity analysis can reveal the basiscs of brain connectivty. Functional connectivity is generaly used for resting state networks and looks for correlational ossilations. Effective connectivity analysis can reveal causal relationships between brain regions. It looks for if one region causes the activation of another and if the cognitive task affects this relationship. Dynamic Causal Models (DCM) is a common method for effective connectivity analysis. DCM starts with defining regions of interest (ROI). Researchers model the connectivity between the regions. The direciton and the effects of cognitive tasks are important in these models. DCM finds the best model depend- 
ing on the fNRI data. We searched for the relationship between the time perception and working memory. We applied visual tasks to healthy individuals and found brain activity in dorsolateral prefrontal, inferior parietal, insular cortex and basal ganglia.But the standart $\mathrm{fMRI}$ analysis do not give information about connectivity. The above mentioned brain regions are taken as ROIs and a DCM analysis was performed. The results were discussed in terms of "internal clock" and "neural network" hypothesis which of time perception mechanisms.

Keywords: fMRI, effective connectivity, DCM, time perception

\section{C-044}

\section{Connectivity modeling and dynamic Bayesian networks}

Ulusoy I

Faculty of Electrical and Electronics Engineering, Middle East Technical University, Ankara, Turkey

DTI, fMRI and EEG data are used to model structural, functional and effective connectivity. Anatomical connectivity can be extracted from DTI data however for functional and effective connectivity fMRI and EEG data are used although there are resolution problems. fMRI has a very high spatial resolution but low temporal resolution whereas EEG has just the opposite. In spite of all these shortcomings, EEG and fMRI data is used for brain connectivity modeling by the following approaches: correlation and covariance based methods, information theory, Granger causality, dynamic causal models, graph theory and dynamic Bayesian networks (DBN). These methods may be applied in time or frequency domain. Many of these methods use the assumption that the connectivity among brain regions is stationary and linear. All of the methods, except DCM, are data driven modeling approaches. However, DCM proposes a model and verifies it based on the acquired data. All of the methods, except DBN, are deterministic. Only DBN is a probabilistic approach. With the help of DBN, nonlinear and probabilistic connectivity models can be developed. Brain regions are represented by the nodes of the network and the connectivty among the regions are represented by the directed edges of the network. Nodes and edges are modeled by conditional probabilities. The presence or absence of edges among nodes can be learned from the acquired data and this is known as structure learning. When structure is learned, connectivty model is reached. Many DBN approaches have been proposed but they are still open to further development.

Keywords: connectivity model, effective connectivity, dynamic Bayesian model

\section{C-045}

\section{Brain connectivity networks in neurology and psychiatry}

Bilgiç B

Department of Neurology, Istanbul University, Istanbul Faculty of Medicine, Istanbul, Turkey

Functional brain imaging is one of the main research method used for to investigate the neural basis of perception, cognition and emotion. In the absence of any task, a spontaneously correlated blood oxygen level-dependent (BOLD) signal can be detected in spatially distinct but functionally related cortical and subcortical regions. Resting state of the brain characterized by low-frequency activity consumes approximately $80 \%$ of all energy used by the brain and is called "dark energy of the brain". Based on the resting activity of the brain detected by fMRI, "Intrinsic Connectivity Networks - ICN" have been identified and "Default Mode Network-DMN" is the first of them. Data collected on DMN suggests that it is involved in retrieval of the semantic knowledge and autobiographic memories. Abnormal DMN activity is the very early feature of Alzheimer's disease and this abnormality appears even before the onset of the symptoms, however, other networks such as salience network and sensorimotor network seem to be preserved in the course of the disease. Frontotemporal dementia, another form of neurodegenerative dementias, is strongly correlated with the diminished connectivity in salience network and DMN seems to be preserved or show increased connectivity in the course of the disease. Recently different pattern of network abnormalities have been reported in other neurodegenerative diseases and psychiatric disorders. As a non-invasive method, resting state fMRI has a potential to be used as a biomarker and to identify very early stages (also preclinical) and to track the course of the many neuropsychiatric diseases.

Keywords: connectivity, resting state networks, default mode network, neurodegenerative, neuropsychiatry

Sunday, 3 May 2015

08:30 - 10:30

PANEL 4

Neurogenetics

Chairs:: Prof. Dr. Aslı Tolun \& Prof. Dr. Uğur Özbek

\section{C-046}

\section{Modes of inheritance and importance of genetics in health}

Turanlı Tahir E

Faculty of Science and Letters, Department of Molecular Biology and Genetics, Istanbul Technical University, Istanbul, Turkey

Modes of inheritance attempt to describe the transmission patterns of genetic based traits and diseases from one generation to another. The three main types of inheritance modes are monogenic (Mendel mode), complex and mitochondrial. Muscle diseases and neurodegenerative diseases are examples of monogenic diseases common in our country. Although genetics have an important role in some types of brain cancers, they show complex inheritance. Most of the mitochondrial mutations cause severe neurological diseases; the defect is usually caused by a new mutation in the mother. New diseases in our country, where consanguineous marriages common, are more frequently observed. Variable penetrance, expression, locus and allelic heterogeneity, epistasis impedes the diagnosis of the patients within the pedigrees, which poses a problem for understanding of the correct inheritance pattern. Complex inherited diseases are common, 
caused by genes and environmental interactions. Some neurological and psychiatric disorders fall into this group. These disorders do not usually follow a strict inheritance mode, however dominant or recessive forms are observed in some families. For example, $5 \%$ of Parkinson's disease patients show a familial inheritance due to mutations in PARK genes. GWAS have led to identification of many susceptibility genes and variations in complex disorders including Alzheimer's disease and multiple sclerosis. These diseases usually have multiple genes and alleles contributing to predisposition. Since genome variations in common disease don't explain all the heritability, missing heritability, epigenetic and post-translational modifications are important research topics. Some SNPs are associated with drug response, interactions and metabolic pathways through pharmacogenomics research. SNPs in cytochrome $\mathrm{P} 450$ gene are associated with response to treatment in of most frequently used antipsychotic drugs and other common drugs. Similarly, nutrigenomics analyses associations between food response and genetic variations. Drug or food response also show complex inheritance and such susceptibility types variations have varying frequencies among populations.

Keywords: monogenic disease, complex disease, genomic variations

\section{C-047}

\section{Gene identification strategies with relevant examples from Turkey in neurological disorders}

\section{Uğur SA}

Department of Genetics, Istanbul University, Institute of Experimental Medicine, Istanbul, Turkey

Identification of genes associated with single gene disorders that show Mendelian inheritance patterns have been accelerated upon completion of the Human Genome Project and application of high throughput next generation technologies in genetic research. This capability has long been an impulsion on genetic analysis of complex disorders as well. Neurological disorders including epilepsy, intellectual disability, muscle dystrophy and peripheral neuropathy together with very well known neurodegenerative disorders such as Alzheimer's and Parkinson's diseases have served as attractive models to study common and rare genetic variants underlying the complex patterns determining the development and function of the nervous system. There is an increased incidence for rare neurological disorders and also rare subtypes of common disorders in Turkey due to a relatively high rate of consanguineous marriages and presence of families having multiple children traditionally. This presentation focuses on familial gene identification strategies in rare neurological disorders using linkage analysis, homozygosity mapping and/or exome sequencing with real examples from Turkey. These rare disease studies have the potential to unravel individual components of complex neurological processes, which will eventually serve as effective diagnostic and therapeutical targets.

Keywords: linkage analysis, exome sequencing, homozygosity mapping, rare neurological disorders

\section{C-048}

\section{Neurogenetics consultancy}

Özbek U

Institute of Experimental Medicine, Istanbul University, Istanbul, Turkey

Genetic consultancy takes place between a genetic consultant and an individual affected from an inherited disorder. The necessity for genetic consultation occurs when diagnosed condition runs in a family or a clinically suspicious undiagnosed condition inherited within a family is subjected to genetic testing. Neurological conditions take up $50 \%$ of the conditions subjected to genetic consultation. The steps taken for a patient with a neurogenetic condition includes; clinical evaluations, recording familial history, providing pre-genetic consultancy, performing genetic testing and reporting after final evaluations. These final reports include evaluations for the index patient as well as any risks calculated for the patient's family members. Genetic tests will provide important results for diagnosis. A patient having a positive family history, a disorder similar to a known genetic condition, a chronic and progressive prognosis, parental consanguinity and/or an ethnicity with a high risk for neurogenetic disorder(s) provide valuable cues for necessity of genetic consultancy. Autosomal dominant, recessive, $\mathrm{X}$-linked and mitochondrial inheritance types usually marks forfamilial history. On the other hand, isolated or sporadic cases along with family members acquiring a non-genetic condition due to environmental conditions do not require genetic consultation. 2 people in a population have approximately one nucleotide change (variation) in each 1000 DNA bases. These variations detected more than $1 \%$ in a population are referred as polymorphisms. The protein coding regions of the genome are under strict control and thus throughout evolution are well preserved and protected from mutations. Therefore, genetic variations usually reside in the non-coding genome. The importance of single nucleotide polymorphisms (SNPs) associated with disorders in diagnosis and prognosis will be highlighted in the presentation.

\section{C-049}

Diagnosis projects by using next-generation sequencing in undiagnosed patients

\section{Onat OE, Özçelik T}

Department of Molecular Biology and Genetics, Bilkent University, Ankara, Turkey

In the last three decades, phenotype-driven approaches have been successful especially in discovering the genetic causes of the monogenic disorders. These approaches include recognition of phenotypic and radiological features, analysis of biopsies and metabolites measurements or specific tests such as karyotyping, comparative genomic hybridization, candidate single gene tests and gene-panel tests. However, they are not adequate in diagnosing rare disorders that are not discrete clinical entities, have variable clinical manifestations or are clinically very similar. Genetic disorders are estimated to occur 40-82 times per 1000 living births, and this rate increases to $8 \%$ if all congenital anomalies included. Because many of these cases are unable to be specifical- 
ly diagnosed, next generation sequencing techniques are used for the genotype-driven molecular diagnosis of rare or common multigenic genetic disorders in the very recent years. These techniques able to identify Mendelian or de novo point mutations, insertions/deletions, structural variations, and copy number changes. The first diagnostic study, published in 2012, genetically diagnosed six of 12 undiagnosed patients with exome sequencing. A study published in 2013, with 250 probands of whom $80 \%$ were children with neurological phenotypes, demonstrated $25 \%$ success in molecular diagnosis. Another study conducted on 2000 patients and 814 trios that consulted California University, Clinical Genomics Center in 2012-2014 revealed that exome sequencing was able to diagnose $25.2 \%$ and $26 \%$ of the undiagnosed patients, respectively. Additionally, $31 \%$ of 1133 children referred to 24 services of National Health Center around UK and Ireland were diagnosed molecularly and meta-analysis of these results revealed 12 novel genes associated with these diseases. Overall, using genotype-driven approaches, increased the diagnosis rate to $25-50 \%$ in patients who cannot be diagnosed by phenotype-driven approaches as well as provided identification of novel genes associated with the disease phenotypes.

Keywords: molecular diagnosis, next-generation sequencing

Sunday, 3 May 2015

11:15 - 12:00

CONFERENCE 10

Trafficking in neuronal cells: new evidences in Alzheimer's disease Chair: Prof. Dr. Gülgün Şengül

\section{C-050}

Trafficking in neuronal cells: new evidences in Alzheimer's disease

Di Luca $M$

Department of Pharmacological and Biomolecular Sciences, University of Milan, Milan, Italy

Alzheimer's disease (AD) is emerging as the most prevalent and socially disruptive illness of aging populations. Genetic and pathological evidence strongly supports the amyloid cascade hypothesis, which states that Amyloid $\beta$ (A $\beta$ ) has an early and crucial role in $\mathrm{AD}$. $\mathrm{A} \beta$ is liberated from the amyloid precursor protein (APP) by BACE and $\gamma$-secretase activity. Alternatively, APP is cleaved within the A $\beta$ domain by ADAM10, which prevents $A \beta$ formation. In addition to $A \beta$, synapse loss has a central role in $\mathrm{AD}$ pathogenesis, rather than just a consequence of cell death. Synapse loss represents an early insult that advances with disease and dendritic spine loss is seen in several $\mathrm{AD}$ models. Therefore, it is fundamental to study the mechanisms underlying the crosstalk between $A \beta$ and synaptic function and failure. Here, we provide new evidences on local and long-distance trafficking pathways relevant for $\mathrm{AD}$ pathogenesis. At a local level, we described the mechanisms responsible for ADAM10 forward trafficking and endocytosis, that are important for the A, production and spine remodelling. We provided a mechanism according to which ADAM10 local trafficking from dendritic Golgi outposts to the postsynaptic density is mediated by SAP97 and is under the control of PKC-dependent phosphorylation of SAP97.
On the other hand, ADAM10 endocytosis is mediated by activity-regulated association of ADAM10 with the clathrin adaptor AP2 complex. Moreover, we showed that ADAM10 local trafficking mechanisms are impaired in $\mathrm{AD}$ patients at the earlier stages of the disease. With regards to long-distance trafficking, we have identified the Ring Finger Protein 10 (RNF10), a novel synaptonuclear protein messenger, which directly associates with the GluN2A subunit of NMDA receptors. Activation of synaptic GluN2A-containing NMDARs leads to translocation of RNF10 from distal dendritic segments and dendritic spines to the nucleus and results in the induction of expression of newly identified RNF10 target genes, implicated both in spine morphology and in $\mathrm{AD}$ pathogenesis. Furthermore, RNF10 expression is altered in $\mathrm{AD}$ patients' hippocampus at the earlier stages of the disease and A, oligomers trigger NMDA-induced RNF10 translocation from the synapse to the nucleus, indicating the involvement of RNF10 mediated pathway in $\mathrm{AD}$ pathogenesis.Taken together these results provide a complete picture of the network of interacting pathways leading to $\mathrm{AD}$ that is mandatory for the development of successful therapies.

Sunday, 3 May 2015

14:00 - 14:45

CONFERENCE 11

Principles of astrogliopathology

Chair: Prof. Dr. Ahmet Ayar

\section{C-051}

Principles of astrogliopathology

Verkhratsky A

Faculty of Life Sciences, University of Manchester, Manchester, UK

The common and prevailing point of view considers neurones as main substrates of pathological progression of neurological diseases. Today, however, we know that integration and information processing in the brain occurs though close interactions of two cellular circuits represented by neuronal networks embedded into internally connected astroglial syncytium. Our understanding of glial function changed dramatically over last two decades. This change concerns the whole concept of how the brain is organized, and how the development, life and death of neural circuits are controlled. There is compelling evidence demonstrating that these are the astrocytes that are creating the compartmentalisation in the CNS, and these are the astrocytes that are able to integrate neurones, synapses, and brain capillaries into individual and relatively independent units. Astroglial syncytia allow intercellular communication routes, which permit translocation of ions, metabolic factors and second messengers. The resulting potential for parallel processing and integration is significant and might easily be larger, but also fuzzier, than the binary coded electrical communication within the neuronal networks. The neuronal-glial circuitry endowed with distinct signalling cascades, form a "diffuse nervous net" suggested by Golgi, where millions of synapses belonging to very different neurones are integrated first into neuronal-glial-vascular units and then into more complex structures connected through glial syncytia. These many levels of integration, both morphological and functional, 
presented by neuronal-glial circuitry ensure the spatial and temporal multiplication of brain cognitive power. Neuroglial cells are intimately involved in all forms of neurological diseases and this are neuroglia, which, to a very large extent, determine the progression and outcome of neuropathological process. Astrocytes are specifically involved in various neurodegenerative diseases including Alzheimer's disease, Amyotrophic lateral sclerosis, Parkinson's disease and various forms of dementia. Recent evidence suggest that early stages of neurodegenerative processes are associated with atrophy of astroglia, which causes disruptions in synaptic connectivity, disbalance in neurotransmitter homeostasis and neuronal death through increased excitotoxicity. At the later stages astrocytes became activated and contribute to neuro-inflammatory component of neurodegeneration.

Sunday, 3 May 2015

15:00-15:45

CONFERENCE 12

Glymphatic system

Chair: Prof. Dr. Ramazan Bal

\section{C-052}

\section{Glymphatic system}

Akdemir G

Department of Neurosurgery, Selçuk University, Faculty of Medicine, Konya, Turkey; Department of Medicine, Verkman Laboratory for Cell \& Membrane Biophysics, University of California San Francisco (UCSF), San Francisco, CA, USA
Lymphatic system facilitates the clearance of proteins and fluid of intercellular space, a role critical to organs function. The brain, despite its high level organization and metabolic activity, there is not known classical lymphatic system. In the brain small molecules and substrates for specific blood brain barrier transporters are cleared from interstitial space to cerebrospinal fluid space, where they are eliminated via arachnoid granulations and superior sagittal sinus. In two years studies show that brain anatomic pathway that facilitates the exchange of interstitial fluid (ISF) and cerebrospinal fluid (CSF) and eliminated of interstitial solutes from brain. There are three pathways: 1 . Para-arterial CSF influx route 2. Para-venous ISF clearance route 3. Trans-parenchymal pathway; dependent astrocytic aquaporin 4 (AQP4) water channel Subarachnoid CSF rapidly enters the brain parenchyma along paravascular spaces - the Virchow-Robin spaces- then reached the basal lamina of the brain capillary bed. The AQP4 is expressed in perivascular astrocytic end feet. This paravascular AQP4 may facilitate the convective bulk flow of fluid from para-arterial CSF influx pathway through the interstitium, and along the para-venous clearance route. Some researcher and we evaluated CSF pathway in AQP4 knockout mice, CSF influx trough the brain parenchyma was reduced. The critical role that perivascular AQP4 plays in the clearance of interstitial solutes soluble betaamyloid, changes in AQP4 mislocalization after traumatic brain injury, vascular dementia may have critical implications fort he pathogenesis of clinical status. 


\title{
Oral Presentations
}

\author{
(S-001 - S-027)
}

\section{0-001}

\section{An in vitro ischemic neurodegeneration model on rat} hippocampal slices and neuroprotective practices

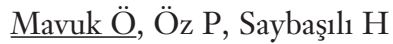

Biomedical Engineering Institute, Bogazici University, Istanbul, Turkey

Objective: Ischemia-related neurodegeneration is a common symptom of neurodegenerative diseases. Our study aims developing an in vitro ischemic neurodegenerative model that can be conducted on $200 \mu \mathrm{m}$ brain slices and analyzed quantitatively through histologic techniques.

Methods: To develop an in vitro ischemic neurodegenerative model, we applied oxygen-glucose deprivation (OGD) on acute hippocampal slices obtained from young adult rats. Histology is used to investigate the morphological changes caused by OGD and neuroprotective factors (baclofen, memantine, Lcarnitine, low temperature). Since our primary technique is whole-cell patch-clamp, histology was performed on $200 \mu \mathrm{m}$ brain slices too. To detect the regional neurodegeneration quantitatively, the stratum pyramidale (SP) width of CA1 is analyzed and changes are expressed as the percentage of mean in each group, normalized with the control group means.

Results: Previous studies report selective vulnerability of CA1 to ischemia-related neurodegeneration. Our study also indicates a selective decrease in mean width of CA1 SP for OGD durations more than 15 minutes. L-carnitine neuroprotective effect is first observed at $10 \mu \mathrm{M}$. Baclofen is effective after $25 \mu \mathrm{M}$ and the effect doesn't change for higher doses. Memantine is effective at $5 \mu \mathrm{M}$ and onwards. It is found that incubation at $+4{ }^{\circ} \mathrm{C}$ during OGD application $(60 \mathrm{~min})$ has an effect equal to the best results of neuroprotective agents. Applications of these agents as combinations of lower doses are also shown to be effective.

Conclusion: Neurodegenerative effect on CA1 can be partially prevented by baclofen, memantine, L-carnitine and low temperature. Our study is continued by electrophysiological experiments.

Keywords: oxygen-glucose deprivation, hippocampus, baclofen, memantine, L-carnitine

\section{0-002}

The association between tau hyperphosphorylation and PIN1 expression in primary cortical neurons

$\underline{\text { Metin D }}$, Gezen-Ak D, Dursun E, Atasoy IL, Y1lmazer S, Öztürk M

Istanbul University, Cerrahpaşa Medical Faculty, Department of Medical Biology, Istanbul, Turkey
Objective: Tau hyperphosphorylation is associated with the inhibition of protein phosphatase 2A (PP2A). Peptidyl-prolyl cis-trans isomerase (Pin1) regulates the phosphorylation of Ser/Thr sites of tau protein; the dephosphorylation of these sites is inhibited by PP2A. Pin 1 is co-localized with phosphorylated tau aggregates in the brains of Alzheimer's patients. In this study, a tauopathy model was established by treating primary cortical neuron cultures with okadaic acid (OKA), which in turn triggers the hyperphosphorylation of tau.

Methods: Cortical neurons were obtained from embryonic day 16 (E16) Sprague Dawley rat embryos. The neurons were treated with $25 \mathrm{nM}$ OKA on day 7 of culture, and the control of PP2A inhibition via tau phosphorylation was analyzed by western blot. Phosphorylated and non-phosphorylated tau proteins were detected by anti- tau antibodies; Thr231 and Tau-1. Pin 1 mRNA expression was determined by qRT-PCR at the 4th, 8th and 24th hours following treatments.

Results: Tau phosphorylation at Thr231 was increased, and nonphosphorylated Tau-1 was decreased 8 hours following treatment with OKA compared with the untreated control and vehicle groups. Pin $1 \mathrm{mRNA}$ expression levels in cortical neurons at both 4 and 8 hours post-OKA treatment were lower than those of the untreated control and vehicle groups. There were no significant differences for Pin $1 \mathrm{mRNA}$ expression in any of the groups after 24 hours of treatment.

Conclusion: We demonstrate for the first time that OKA might induce tau hyperphosphorylation by reducing the expression of Pin 1 mRNA in addition to suppressing PP2A.

Keywords: cortical neuron, tau hyperphosphorylation, pin1, okadaic acid, PP2A

\section{0-003}

Cyto- and chemoarchitecture of the mouse spinal dorsal horn in 3D using CLARITY

Şengül $\mathrm{G}^{1}$, Liang $\mathrm{H}^{2}$, Paxinos $\mathrm{G}^{2}$

'Department of Anatomy, Ege University Faculty of Medicine, Izmir, Turkey; ${ }^{2}$ Neuroscience Research Australia, Sydney, Australia

Objective: CLARITY is a newly-developed technique developed by Deisseroth's group in Stanford (K. Chung et al. Nature Methods 10:508-513, 2013), and is considered one of the most important advances in neuroanatomy. The dorsal horn of the spinal cord is morphologically heterogeneous neurons varying considerably in size, shape and density. There are ten laminae in spinal cord and over ten different nuclei first described in the cat, later in the mouse, rat, and for the first 
time (Atlas of the Spinal Cord, Sengul, Tanaka, Watson, Paxinos, Elsevier, 2013) in marmoset monkey, rhesus monkey and human. This study was designed to show organization and chemo-architecture of the mouse spinal cord in 3D using CLARITY, to reveal new details and be a guide for further cord studies using this technique.

Methods: C57BL/6J mice were perfused $(n=12)$ perfused with ice cold hydrogel solution, spinal cords cut into $2-3 \mathrm{~mm}$ segments and washed with clearing solution until optically transparent. Ten markers were used for immunostaining. Tissue was imaged using multiphoton microscope.

Results: Calbindin, calretinin, parvalbumin, CGRP, ChAT, serotonin, glycine, GAD67, NOS and GABA revealed details of $3 \mathrm{D}$ organization and chemo-architecture of the dorsal horn laminae and spinal cord nuclei.

Conclusion: Until recently, the only way to visualize the spinal cord in $3 \mathrm{D}$ was to do serial sections and make a reconstruction of these. However, tissue sectioning and subsequent imaging of individual sections have various limitations. Interesting details of organization of mouse spinal dorsal horn anatomy and its chemo-architecture were observed in $3 \mathrm{D}$ for the first time.

Keywords: chemo-architecture, CLARITY, cyto-architecture, mouse, spinal cord

\section{0-004}

Morphometric aspects of the hypoglossal canal as a guide for microneurosurgery

\section{$\underline{\text { Kayhan }}^{1}$, İlhan $\mathrm{P}^{1}$, Ertürk $\mathrm{M}^{2}$, Şengül $\mathrm{G}^{2}$}

${ }^{1}$ Department of Neuroscience, Ege University, Institute of Health Sciences, Izmir, Turkey; ${ }^{2}$ Department of Anatomy, Ege University School of Medicine, Izmir, Turkey

Objective: Surgical management of tumors involving the hypoglossal canal (HC) have challenges. Traditional skull base approaches provide limited access to this region. Evaluating $\mathrm{HC}$ bridging is also important to help understand various pathologic processes, such as dural arteriovenous fistula. This study was undertaken to determine morphometric aspects of human HC in recent Turkish population.

Methods: HC's of 100 dry human occipital bones from the collection of Ege University, School of Medicine Department of Anatomy were evaluated. A digital caliper (accuracy 0.01 $\mathrm{mm}$ ) and a protractor were used take measurements of ten different parameters of HC. Paired t-test was used for assessment of right-left differences.

Results: Bilateral single HC was prevalent over other forms of variation of $\mathrm{HC}$ as observed in 78 occipital bones, as opposed to $\mathrm{HC}$ with unilateral septum in thirteen and bilateral septum in seven skulls. Intracranial HC diamater was on the right $6.34 \pm 1.5 \mathrm{~mm}$, on the left $5.72 \pm 1.26 \mathrm{~mm}(\mathrm{p}<0.05)$. Distances from the intracranial and extracranial ends of $\mathrm{HC}$ to jugular foramen were $9.63 \pm 1.81 \mathrm{~mm}$ and $2.87 \pm 1.25 \mathrm{~mm}$, respectively. Distances from the $\mathrm{HC}$ to the opisthion and basion were
$30.40 \pm 2.93 \mathrm{~mm}, 16.05 \pm 2.01 \mathrm{~mm}$ respectively. The distances from the intracranial end of $\mathrm{HC}$ to posterior margins of $\mathrm{OC}$ were $9.69 \pm 1.39 \mathrm{~mm}$ and distance from $\mathrm{HC}$ to anterior margins of OC $10.74 \pm 1.47 \mathrm{~mm}$.

Conclusion: The present study gives insight into the cranial variants of the $\mathrm{HC}$ and morphometric features related to the $\mathrm{HC}$ in a recent Turkish population for achieving accurate orientation and effective maneuvers during surgical procedures, including safe drilling.

Keywords: hypoglossal canal, morphometry, occipital bone

\section{0-005}

\section{Detecting mirror neuron candidates in F5 area via object level decoding}

Kirtay $M^{1}$, Papadourakis $V^{2}$, Raos $V^{2}$, Oztop $E^{1}$

'Computer Science Department, Ozyegin University, Istanbul, Turkey; ${ }^{2}$ Medical School, Foundation for Research \&Technology- Hellas (Forth), and University of Crete, Heraklion, Greece

Objective: It is known that some neurons in the area F5 of macaque monkeys have visuomotor responses for specific type of grasp execution and observation. Usually the determination of whether a neuron has mirror characteristics is done manually in a heuristic way. In this study, we propose a decoding framework that can be used to mechanistically detect mirror neurons given a set of neural firing.

Methods: Neuronal activations of 68 neurons in the left hemisphere of a Macaque Monkey were used in four different experiment conditions with cylinder, sphere, ring and cube shaped objects. The experiment conditions were 'observation with cue', 'observation without cue', 'object fixation', and 'execution'. To apply regression, the neuronal activations $(\mathrm{X})$ was used as samples from the independent variable and the object ids of $\{1,2,3,4\}$ were used in making up Y, the samples of dependent variable to be predicted. Based on the number of correct predictions, all neurons were ranked for each individual object to observe whether the same neurons could be found both in action observation and action execution conditions.

Results: With the available data we observed that for execution-observation 'with cue condition' 5 neurons (this is 3 in 'without cue condition') that were high performers (>40\% successful prediction vs. chance level of $25 \%$ ) were also found to be high performers in action execution condition.

Conclusion: With this work, we give a mechanistic procedure for detecting object-specific mirror neurons and show that it works with real data.

Bilateral S\&T Cooperation Program Turkey (TÜBITAK113S391) and Greece (MCERA/GSRT - ${ }^{\circ} \mathrm{I}$ I 14DUR OBSERVENEMO).

Keywords: mirror neuron, neural representation, neural decoding 


\section{0-006}

The mechanism of $\mathrm{P} 2 \mathrm{X7}$ receptor mediated neuronal survival in vivo and in vitro

Çağlayan AB, Yalçın E, Beker MÇ, Çağlayan B, Keleştemur T, Öztürk G, Kılıç E

Istanbul Medipol University, Faculty of Medicine, Department of Physiology, Istanbul, Turkey

Objective: $\mathrm{P} 2 \mathrm{X} 7$ receptors $(\mathrm{P} 2 \mathrm{X} 7 \mathrm{R})$ are members of the family of cationic-selective ion channels gated by extracellular ATP and a promising target for the treatment of neurodegenerative disorders. In the present study, we evaluated the effect- and mechanism of $\mathrm{P} 2 \mathrm{X} 7$ receptors on neuronal injury, signaling pathways and calcium toxicity in-vivo and in-vitro.

Methods: Besides analysis of expression patterns of P2X7R invivo and in-vitro, we used focal cerebral ischemia model in mice for the evaluation of neuronal injury, DNA fragmentation and signaling pathways by using receptor inhibitor BBG and activator BzATP. Furthermore, we analyzed the role of $\mathrm{Ca}++$ signaling in the neuroprotective effects of $\mathrm{P} 2 \mathrm{X} 7 \mathrm{R}$ in primary cortical cell by using FURA-2 AM analysis.

Results: In contrast to recent studies, we observed that the cellular expression of $\mathrm{P} 2 \mathrm{X} 7 \mathrm{R}$ is limited to neuronal cells in-vivo and in-vitro. Although the activation of $\mathrm{P} 2 \mathrm{X} 7 \mathrm{R}$ had no toxic effect, inhibition of P2X7R improved neuronal survival significantly, which was associated with increased phosphorylation of survival kinases Akt and ERK-1/2. Furthermore, activation of P2X7 receptor with BzATP increases $\mathrm{Ca}++$ entry into the cortical neurons in-vitro. Inhibition of $\mathrm{P} 2 \mathrm{X} 7$ receptors by $\mathrm{BBG}$ significantly decreases Glutamate- and Thapsigargin- induced $\mathrm{Ca}++$ accumulation and neuronal death.

Keywords: P2X7 receptors, neuronal survival, apoptosis, FURA-2 AM, calcium imaging, thapsigargin, glutamate

\section{0-007}

Role of PACAP, VIP and receptors in rat cerebral cortex in experimental diabetes model

Üner MA, Temel M, Tuğlu Mí, Özkut M, Abulimiti R, Özbilgin K

Deparment of Histology \& Embryology, Celal Bayar University, Faculty of Medicine, Manisa, Turkey

Objective: Diabetics is known to cause brain impairment but entire impairment mechanism in the brain remains unknown. Known as neuroprotectant agents PACAP, VIP and their receptors PAC 1, VPAC 2 were intended to be examined in terms of their neuroprotectant influences in diabetics formed rat model.

Methods: 18 Pieces Wistar rats divided into three groups. First group was divided as con-trol group second group was equipped with fructose and the third group was al-located with STZ. Animals by means of cervical dislocation taken out their brains through decapitation. Following classical tissue follow- up procedure al-located dissections by histochemical methodology were stained with monoclonal anticores of PACAP, VIP, PACP 1 and VPAC 2 by immunohistochemical method and with Hematoxylene-eosine.

Results: At the end of cerebral cortex related tissue samples immunochemical detection in PACAP control cohort found to have been expressed negligibly on the other hand predominantly in STZ applied groups expression proliferation was observed. Also detected PACAP receptors similar with PACAP expressions stain-ing properties were found.

Conclusion: Diabetics is known to cause impairment over the nerve tissue. Tissue damage mechanisms due to hyperglycemia are not known entirely. In this present study, PACAP, VIP and their receptors proliferations in diabetics groups made us to suppose as if these molecules may be responsible in diabetics impairment.

Keywords: brain, fructose, STZ, diabet, PACAP, VIP

\section{0-008}

Protective effects of pterostilbene treatment in diabetic neuropathy

$\underline{\text { Bozkurt }}^{1}$, Keser $\mathrm{H}^{2}$, Köken $\mathrm{EC}^{2}$, Bilgin $\mathrm{MD}^{1}$

'Department of Biophysics, Adnan Menderes University Faculty of Medicine, Aydın, Turkey; ${ }^{2}$ Department of Biophysics, Adnan Menderes University Institute of Health Sciences, Aydın, Turkey

Objective: Pterostilbene, a natural metoxyl derivative of resveratrol, is an antioxidant having anti-inflammatory and antidiabetic effects. Pterostilbene has been reported to have protective effects in neurological, cardiovascular, metabolic and hematologic diseases and to decrease blood glucose levels in diabetes. This study aims to evaluate the neuroprotective effects of low and high dose pterostilbene treatment in experimental diabetic neuropathy.

Methods: Adult male Wistar rats $(\mathrm{n}=30)$ were randomly assigned for control, diabetic, low $(10 \mathrm{mg} / \mathrm{kg})$ and high (40 $\mathrm{mg} / \mathrm{kg}$ ) dose pterostilbene treated diabetic groups. Pterostilbene used in the study was generously provided by Sabinsa Corporation. Diabetes was induced by a single intraperitoneal injection of STZ $(50 \mathrm{mg} / \mathrm{kg})$, and 3 days after the STZ injection the rats having hyperglycemia ( $>300 \mathrm{mg} / \mathrm{dl}$ ) were assigned to be diabetic. Rats in treatment groups received low $(10 \mathrm{mg} / \mathrm{kg} /$ day $)$ and high (40 mg/kg/day) doses of intraperitoneal pterostilbene injection for 7 weeks. Nociceptive tests (tail flick, hot plate) were conducted at $3 \mathrm{rd}$ and 5 th weeks of treatment. Nerve conduction velocities (NCV) and distal latencies were determined in sciatic nerves.

Results: High dose pterostilbene treatment led to a significantly decrement in the diabetes-induced elevated plasma glucose levels $(\mathrm{p}<0.01)$. In addition, the elevated tail flick and hot plate latencies in diabetic group were reduced by pterostilbene treatment, revealing its protection on nociceptive pain perception. Moreover, the decreased NCVs in diabetes were improved by the treatment of both doses of pterostilbene. 
Conclusion: In conclusion, these findings revealed the neuroprotective role of pterostilbene treatment in diabetic neuropathy apart from restoring the normal blood glucose levels.

Keywords: pterostilbene, diabetic neuropathy, nerve conduction velocity, nociceptive tests

\section{0-009}

Decision making and skin resistance as a somatic marker in mesial temporal lobe epilepsy

$\underline{\text { İçellioğlu S}}^{1}$, Deli Ş ${ }^{2}$, Yeni N ${ }^{2}$, Kurtish Yağcı $S^{3}$, Korkmaz B ${ }^{2}$

'Department of Psychology, Istanbul Kültür University, Istanbul, Turkey; ${ }^{2}$ Department of Neurology, Istanbul University Cerrahpaşa Medicine Faculty, Istanbul, Turkey; ${ }^{3}$ Department of Neurosurgery, Istanbul University Cerrahpaşa Medicine Faculty, Istanbul, Turkey

Objective: Iowa Gambling Test (IGT) showed patients with frontal lobe lesions performed well on standard neuropsychological tests, however showed lower net scores on IGT. Somatic marker hypothesis $(\mathrm{SMH})$ proposes that emotional processes are guided by a variety of somatic signals such as skin conductance, especially in decision making. The purpose of this study is to test the SMH and to assess DM performances of patients with mesial temporal sclerosis (MTS) with a control group.

Methods: 22 patients $\left(M_{ \pm} \mathrm{SS}=32.7 \pm 10.998\right.$ for age $)$ and 24 healthy paticipants with similar sociodemographic characteristic $\left(\mathrm{M}_{ \pm} \mathrm{SS}=31 \pm-9.8\right.$ for age) were included. The computerized version of IGT had been used to assess DM. Skin resistance values are recorded as an anticipatory response just before $-2 \mathrm{~ms}$. the participant makes his choice. The effect of deck on anticipatory responses had been analysed by using Univariate ANOVA statistics. Independent samples T-test had been used to compare IGT total net scores between control and epilepsy group.

Results: In control group, variance analysis showed a main effect of deck on anticipatory responses for $\mathrm{AB}$ decks ( $\mathrm{F}$ $(1.184)=4.33 ; \mathrm{p}<0.05)$. No main effect had been found in epilepsy group. Results indicated that the scores were significantly higher for control group $(M=11.6, S D=14.5)$ than for epileptic group $(M=-2.55, S D=17.96),(t(44)=2.95 ; \mathrm{p}<0.05)$.

Conclusion: The control group makes more adventogeous choices than the patient group in IGT. MTS patients showed different SM responses with controls. This result may be comparable with patients having ventromedial frontal lobe lesions.

Keywords: mesial temporal sclerosis, decision making, Iowa Gambling test, somatic marker hypothesis

\section{0-010}

Recording cervical vestibular evoked potentials with a different technical procedure

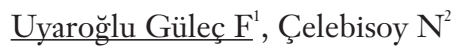

'Department of Neurology, Izmir Tepecik Education and Research Hospital, Izmir, Turkey; ${ }^{2}$ Department of Neurology, Ege University, Faculty of Medicine, Izmir, Turkey
Objective: Cervical vestibular evoked myogenic potential (cVEMP) is a short-latency myogenic response recorded over the sternocleidomastoid muscle (SCM). The aim of this study was to try to make recordings by placing the active electrode on different muscle groups to investigate the origin of the potentials.

Methods: 30 healthy volunteers; 12 women and 18 men, with a mean age of 35 years (18 to 55 years) were included in the study after informed consents were obtained from all. To record the surface EMG activity, an active electrode was first placed on the upper half of the ipsilateral (IL) sternocleidomastoid muscle (SCM) and then IL tibialis anterior (TA) and IL triceps muscles (TCM). The volunteers were asked to rotate the head to activate the SCM maximally during the recordings. The acoustic stimuli were clicks at an intensity of $100 \mathrm{dBnHL}$ (normal hearing level) of 0.1 millisecond duration, delivered at a frequency of $5 \mathrm{~Hz}$ through a headphone unilaterally to each ear. The initial positive/negative polarity of the waveform with peaks was termed p13 and n 23 on the basis of respective latencies. The latencies of peaks $\mathrm{p} 13$ and $\mathrm{n} 23$ and peak-to-peak amplitude of p13-n23 were measured.

Results: In our study cVEMPs were recorded in $100 \%$ of the subjects from the ipsilateral SCM, TCM and TA muscles. Configurations and p13, n23 latencies of cVEMP were not different in all different sides. Median p13, n23 latencies and p13/n23 amplitudes of the responses recorded from three different sites. Amplitudes recorded from SCM were larger than TCM and TA muscles.

Conclusion: The conventional method for recording the VEMP involves measuring EMG activity by surface electrodes placed over the tonically-activated SCM muscles. Responses recorded from different active sites showed nearly the same features which is difficult to explain with the known electrophysiological criteria. Further studies involving different sites must be performed to be more confident about the origin of the potentials.

Keywords: vestibular evoked myogenik potantials, cVEMP, vestibulocollic reflex

\section{0-011}

\section{Comparison of excitability of left and right thumbs by threshold tracking}

Burat $\dot{I}^{1}$, Pala S${ }^{2}$, Korkmaz $M^{2}$, Özmen $G^{2}$

${ }^{1}$ Department of Biophysics, Basic Medical Science, Necmettin Erbakan University, Meram Medical Faculty, Konya, Turkey; ${ }^{2} 2$ nd Grade Student, Necmettin Erbakan University/ Meram Medical Faculty, Konya, Turkey

Objective: Conduction velocity and amplitude calculation from compound muscle action potentials (CMAPs) recorded from thumb which is innerved by median nerve is a frequently used technique in clinic. But there isn't any study that is testing the excitability of thumb branch of median nerve by recording compound nerve action potentials (CNAPs). With 
this study, we aimed to investigate the possible excitability differences between left and right thumb branches of median nerve by recording CNAPs'.

Methods: CNAPs of 6 women and 6 men voluntteers which have ages between 18-24 were recorded. Median nerve was stimulated at wrist by using non-invasive threshold tracking method and CNAP responses were recorded from thumb with ring electrodes. Threshold electrotonus data were obtained by application of over threshold $1 \mathrm{~ms}$ puls couple following $100 \mathrm{~ms}$ subthreshold polarizing current so the excitability of nerve could be recorded along $100 \mathrm{~ms}$.

Results: Recordings from righthanded volenteers for $\% 40$ target has shown that there is significant difference $(\mathrm{p}<0.05)$ in 15 th and 20th miliseconds of depolarizing threshold electrotonus values. Superexcitability, subexcitability, strenght-duration time constant and rheobase parameters didn't show any significant change.

Conclusion: This phase (S1) of electrotonus curve reflects fast $\mathrm{K}+$ channels' activity. These findings for right thumb shows that there is more excitability increase and fast $\mathrm{K}+$ channels have less conductivity. These findings are valid for only moderate and relatively slow conducting fiber so that they gathered from \% 40 electrotonus data. Consequently, there isn't any difference for $\mathrm{Na}+$ channels and rectifying $\mathrm{K}+$ channels activity between left and right thumb branches of median nerve.

Keywords: median nerve, threshold tracking method, compound action potential

\section{0-012}

\section{Effects of autapse on the transsmission of weak localized rhythmic activity}

\section{Baysal $V^{1}$, Yilmaz E ${ }^{1}$, Özer $M^{2}$}

${ }^{1}$ Department of Bioelectronic, Department of Biomedical Engineering, Bülent Ecevit University, Zonguldak, Turkey; ${ }^{2}$ Department of Electronic, Department of Electrical and Electronics Engineering, Bülent Ecevit University, Zonguldak, Turkey

Objective: In this paper, the effects of autapse (a synapse formed between axon and dendrite of same neuron) on the transmission of weak rhythmic activity are investigated depending on ion channel noise in scale-free networks. We assumed that a neuron which has the lowest degree has an autapse. By applying weak signal to this neuron (thereafter called pacemaker), the transmission of its activity across the whole network is investigated.

Methods: In this study, a scale-free network composed of 200 neurons is analyzed. Each neuron is modeled with HodgkinHuxley equatios that reflects the behaviors of a biologic neuron. As a noise source, the ion channel noise stemming from random open-close fluctuations of ion channels is used. To quantitatively measure the transmission of weak localized pacemaker activity in the network, Fourier coefficient (Q) is used.

Results: Firstly, transmission of weak rhythmic activity is investigated by considering that the pacemaker neuron doesn't have autapse. For different values of average degree (kavg) of network and coupling strength ( $\varepsilon 0)$ among neurons, Q is calculated. Maximal transmission is obtained for $\mathrm{kavg}=12$ and $\varepsilon 0=0.05$. Besides, the presence of an optimal channel noise intensity ensuring the best transmission of weak rhythmic activity is obtained. For these optimal parameters, effects of autapse on the transmission of weak rhythmic activity are studied. Obtained results indicate that for the best transmission of the weak rhythmic activity autapse should have the delay time $\tau=20 \mathrm{~ms}$ and coupling strength $\kappa=0.25$.

Conclusion: As a result, autapse has prominently increases the transmission of weak rhythmic pacemaker activity when delay time and autaptic coupling strength have their optimal values.

Keywords: scale-free network, autapse, pacemaker

\section{0-013}

The protective effects of obestatin on oxidative brain damage of rats with epilepsy

Koyuncuoğlu T ${ }^{1}$, Üren D², Yıldırım Ç ${ }^{2}$, Atal SS ${ }^{2}$, Vıdıklar C ${ }^{2}$, Yılmaz $\mathrm{H}^{2}$, Akakın $\mathrm{D}^{3}$, Yüksel $\mathrm{M}^{4}$, Yeğen Çağlayan $\mathrm{B}^{1}$

'Department of Physiology, Marmara University School of Medicine, Istanbul, Turkey; ${ }^{2}$ School of Medicine, Marmara University, Istanbul, Turkey; ${ }^{3}$ Department of Histology and Embryology, Marmara University School of Medicine, Istanbul, Turkey; ${ }^{~}$ Vocational School of Health Related Professions, Marmara University, Istanbul, Turkey

Objective: In generalized epilepsy, increased generation of reactive oxygen metabolites (ROM) triggers convulsions by inactivating glutamine synthase and glutamate decarboxylase, increasing glutamate and decreasing GABA. The present study was planned to elucidate effects of obestatin treatment on severity of seizures, memory performance and brain injury.

Methods: Following a learning-trial using passive-avoidance test, Wistar male rats $(\mathrm{n}=36)$ were injected with saline or obestatin (1 microgram $/ \mathrm{kg}$, intraperitoneally, ip) and $30 \mathrm{~min}$ later pentylentetrazol (PTZ; $45 \mathrm{mg} / \mathrm{kg}$; ip) was injected to induce seizures. In the control group PTZ was not administered. Seizures were video-taped and evaluated by using Racine's scoring (0-5) method. Before rats were decapitated on 72nd hour of PTZ injection, memory performances were evaluated by passive-avoidance test. Brain malondialdehyde (MDA), glutathione (GSH) levels, myeloperoxidase (MPO) activity and chemiluminescence, showing generation of ROM, were measured and histopathological analysis was made. ANOVA and Student's t tests were used for statistical analysis.

Results: Generation of tonic-clonic seizures was reduced in obestatin-PTZ group (25\%) as compared to saline-PTZ (58.3\%) and seizure scores were lower $(\mathrm{p}<0.01)$. Compared to control group, reduced memory performance, increased brain MDA, luminol/lucigenin chemiluminescence, MPO activity $(\mathrm{p}<0.01$ $0.001)$ were reversed by obestatin $(\mathrm{p}<0.05)$. Brain GSH content in both PTZ groups was similar and higher than the control group. Microscopically, neuronal damage observed in the cortex and hippocampus was alleviated in obestatin-treated PTZ group.

Conclusion: Obestatin, which reduced the severity of PTZinduced seizures, improved memory dysfunction and neuronal 
damage, appears to act by inhibiting the generation of ROM, neutrophil infiltration and oxidative damage.

Keywords: epilepsy, oxidative stress, obestatin, memory

\section{0-014}

Effects of mesenchymal stem cell on wound healing of neuroblastoma cell line

$\underline{\text { Abulimiti } \mathrm{R}^{1}}$, Aydemir I $\mathrm{I}^{1}$ Özkut $\mathrm{M}^{1}$, Firat $\mathrm{F}^{1}$, Saygılı $\mathrm{S}^{1}$, Vural $\mathrm{K}^{2}$, Tuğlu $\mathrm{Mi}^{1}$

'Department of Histology-Embryology, Celal Bayar University Faculty of Medicine, Manisa, Turkey; ${ }^{2}$ Department of Pharmacology, Celal Bayar University Faculty of Medicine, Manisa, Turkey

Objective: Investigate the effects of bone marrow mesenchymal stem cells (BMSCs) on the wound healing of mouse neroblastoma cell line $(\mathrm{Na} 2 \mathrm{~B})$ in culture through apoptosis and oxidative stress.

Methods: Na2B cells were differenciated into neurons in culture. BMSCs obtained from Wistar rats and their two day semiconflent meduim were collected as niche and the cells were used for treatment. Na2B cells divided into wounded group (WG), WG treated with niche $(W G+N)$, BMSC $(W G+S C)$ or BMSC combined with niche $(\mathrm{WG}+\mathrm{N}+\mathrm{SC})$ groups. Wounds were created crossshaped by a pipet. Behavior of cells observed by inverted microscope. immunohistochemistry (IHC) staining of eNOS, iNOS, TGF-, 1 and TUNEL staining were applied. Healing scores and IHC parametres stated as the percentage of positive cells.

Results: After 5 days of healing period WG showed \%42, $\mathrm{WG}+\mathrm{N} \% 62, \mathrm{WG}+\mathrm{SC} \% 74$ and $\mathrm{WG}+\mathrm{N}+\mathrm{SC} \% 84$ recovery. We observed that eNOS staining is obvious near the wounded area compare to the rest and treatment reduced the eNOS staining significantly. We demonstrated similar iNOS staining but a bit less compare to the eNOS. Wounded groups showed increased TUNEL staining. TGF-,1 showed increaced staining in treatment groups.

Conclusion: Stem cells and niches have positive effects on experimental wound healling of neural cells separately but more effective used combined. We demonstrate that the healing effects for oxidative stress is via eNOS, iNOS intermediated by TGF-, 1 and by reducing apoptosis. These results indicate that stem cell therapy may affect the neural cell regenaration in vivo positively and may beneficial for nerve system disease as well.

Keywords: bone marrow mesenchymal stem cell, neuroblastoma cell line, apoptosis, wound healing, oxidative stress

\section{0-015}

Moderate neurotoxic effects of local anesthetics on neurons differentiated from stem cells

Tuğlu Mi, Sönmez P, Özkut M, Aydemir I, Firat F, Saygilı S, Ablumiti R

Department of Histology and Embryology, Celal Bayar University Faculty of Medicine, Manisa, Turkey
Objective: Local anesthetics (LA) are used clinically to provide perioperative pain control in many pathologic conditions such as epidural anesthesia. Large concentrations, continuous application and long exposure time can cause neurotoxicity. The aim of this study is to determine the mechanism of toxicity in relation to neurite inhibition, oxidative stress and apoptosis.

Methods: Rat bone marrow stromal stem cells (BMSSC) were induced to differentiate and generate neurites in the culture medium combined with epidermal growth factor and fibroblast growth factor. The culture medium was removed and replaced with serum-free medium containing tetracaine at dilutional concentrations prior to neurite measurement. The cell viability, MTT and toxicity were measured with neurite inhibition. Cells were evaluated for iNOS, eNOS and apoptosis by immunohistochemical staining. Statistical analysis was performed with the scoring.

Results: LA increased neurite inhibition at IC 50 level and it showed toxicity by increasing the number of positive cells by marking of iNOS, eNOS and apoptosis.

Conclusion: LA show toxic effects depending on the dose of repeated use although the use of LA is absolutely reliable and it shows toxicity by neurite inhibition and apoptosis caused by oxidative stress. This means that LA can also damage rather than the benefits and could be a problem in the long term.

Keywords: local anesthetic, stem cell, neurotoxic effect, oxidative stress, apoptosis

\section{0-017}

The effects of adipose derived stem cell application on peripheral nerve regeneration in rats

Kocacan $\mathrm{M}^{1}$, Erdoğan $\mathrm{E}^{1}$, Selimoğlu N${ }^{2}$, Solmaz $\mathrm{M}^{1}$, Tosun $Z^{2}$, Ünlükal $N^{1}$, Yilmaz $M^{1}$

'Department of Histology and Embriyology, Faculty of Medicine, Selcuk University, Konya, Turkey; ${ }^{2}$ Department of Plastic and Reconstructive Surgery, Faculty of Medicine, Selcuk University, Konya, Turkey

Objective: Peripheric nerve injury is an important problem that causes serious morbidity. There are various ways (end to end anastamosis, graft) of restoration in peripheric nerve damage. Synthetic or natural materials are used. In spite of having some disadvantages autograft treatment is still first treatment method. Adipose tissue is a mesodermal based complex tissue containing adipocytes, pre-adipocytes, microvascular endothelial cells, smooth muscle cells, and stem cells. Stem cells are easy to obtain. In our work, we investigated the effects of using adipose derived stem cell to traditional methods on nerve regeneration.

Methods: 64 rats are divided into 8 groups: primary sture, primary sture and stem cell, autograft, autograft and stem cell, disection, disection and stem cell, untreated and control. After 6 weeks, in order to evaluate functional recovery, foot print test (SFI) was made,Samples were stained S-100, PGP 9,5 and DAPI for fluorescence and confocal microscope to evaluate general structure, axon organization and ratio, Schwann morphology and ratio, vascularition, and inflammation condition. 
Results: Primary sture and stem cell, autograft and stem cell groups have increased axon ratio and functional recovery was better. Also there was no smiler effects in disection group. No relation was determined between Schwann cell ratio and stem cell. There was no effect of stemm cells on vascularition, fibrosis and inflammation.

Conclusion: Positive effects of adipose tissue derived stem cells on functional and morphological recovery were determined and these positive effects of stem cells on nerve regeneration are related to secreted sitokins, growth factors and multipotency.

Keywords: peripheral nerve regeneration, adipose tissue derived stem cell

\section{0-019}

\section{Uridine increases pyrimidinergic transmission in rat brain}

Cansev $\mathrm{MH}^{1}$, $\underline{\text { Ulus } \mathrm{IH}^{2}}$, Wurtman $\mathrm{RJ}^{3}$

'Department of Pharmacology, Uludag University Medical School, Bursa, Turkey; ${ }^{2}$ Acibadem University, Medical Faculty, Department of Pharmacology, Istanbul; 3Department of Brain and Cognitive Sciences, Massachusetts Institute of Technology, Cambridge, UK

Objective: Uridine is the principal circulating pyrimidine nucleoside in humans and its nucleotide products uridine-5'diphosphate (UDP) and uridine-5'-triphosphate (UTP) are ligands to P2Y receptors. We now showed that UDP and UTP, as well as uridine, are detectable in dialysates of striatal ECF obtained from freely-moving rats.

Methods: Male rats (300-350 g) anesthetized and microdialysis probe was placed into rthe left striatum 24-h before the experiments. The striatum perfused with the saline solution containing ectonucleotidase inhibitor to prevent breakdown of nucleotides. In some experiments perfusion medium contained high $\mathrm{KCl}(52 \mathrm{mM})$ or TTX $(0.001 \mathrm{mM})$. Uridine, UDP UTP and acetylcholine were assayed by HPLC system.

Results: Mean brain uridine, UDP and UTP levels were $25 \pm 2$, $421 \pm 29$ and $225 \pm 14.6 \mathrm{pmol} / \mathrm{mg}$ tissue, respectively. The basal uridine, UDP and UTP levels in striatal dialysates were 7800, 312 and $181 \mathrm{fmol} / 30 \mathrm{~min}$, respectively. In response to intraperitoneal (i.p.) administration of uridine $(0.1-1 \mathrm{mmol} / \mathrm{kg})$, both brain and dialysate uridine, UDP and UTP levels increased in a dose-dependent manner. Uridine, UDP and UTP levels in the dialysates increased by about 1.7-1.8 fold by high KCL. TTX reduced striatal ECF uridine UDP and UTP levels by about $61-$ $76 \%$. Uridine ( $1 \mathrm{mmol} / \mathrm{kg}$, i.p.) enhanced the striatal ECF concentrations of acetylcholine which was accompanied by increased levels of DAG and IP3 in the brain. The enhanced cholinergic neurotransmission is blocked by pretreatment with suramin.

Conclusion: These data show that existence of pyrimidinergic system in brain and uridine increase pyrimidinergic neurotransmission.

Keywords: uridine, UDP, UTP, pyrimidinergic system

\section{0-020}

Uridine and its nucleotides increase synsaptic proteins and synaptogenesis

Ulus IH

Acıbadem University, Medical Faculty, Department of Pharmacology, Istanbul, Turkey

Objective: Aim of the study was to determine effects of uridine and its nucleotides on synaptogenesis.

Methods: In vitro: PC12 cells were maintained in DMEM supplemented with $10 \%$ fetal bovine serum at 37 oC, $5 \%$ CO2 $+95 \%$ O2. Cells were differentiated for 4 days in medium containing $20 \mathrm{ng} / \mathrm{ml}$ Nerve Growth Factor (NGF) and 1\% fetal with or without test compounds. Uridine (0-0.20o mM), UMP (0-200 $\mathrm{mM})$, UDP $(0-0.020 \mathrm{mM})$ or UTP $(0-0.020 \mathrm{mM})$ was added into the medium. At the end of the period the media were aspirated, cells were scraped in ice-cold water containing protease inhibitors. Synaptic proteins were analysed by Wester Blot. In vivo: Rats or gerbils were given either a control or a UMP-containing $(0.5 \%)$ diet for 3-4 weeks and their brains were harvested and assayed for phospholipids, synaptic proteins and for dentritic spine density.

Results: Incubation differentiated PC12 cells with various doses of uridine, UMP, UDP and UTP resulted with a significant elevations in PSD-95, synapsin, NF-70 and NF-150 levels. Presence of suramin $(0.030 \mathrm{mM})$ or PPADS $(0.030 \mathrm{mM})$, blocked the increases in PSD-95, Synapsin-1, NF-70 or NF150 induced by uridine $(0.100 \mathrm{mM})$, UMP $(0.100 \mathrm{mM})$, UDP $(0.010 \mathrm{mM})$ or UTP $(0.010 \mathrm{mM})$. Dietary supplemntation of UMP $(0.5 \%)$ for 3-4 weeks increased phospholipids, synaptic proteins and dentritic spine density in rat and gerbil brains.

Conclusion: These data show that uridine and its nucleotides stimulate synaptogenesis.

Keywords: uridine, UDP, UTP, synaptogenesis

\section{0-021}

\section{The effect of electromagnetic fields (EMF) on the neuroblastoma cell line behavior during in vitro wound healing}

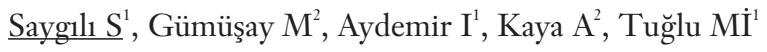

'Department of Histology and Embriyology, Celal Bayar University Faculty of Medicine, Manisa, Turkey; '2Department of Electrical Electronics Engineering, Izmir Katip Celebi University Faculty of Engineering and Architecture, Izmir, Turkey
\end{abstract}

Objective: In our study, we aimed to investigate the effect of pulsed radiofrequency electromagnetic field (PEMF) and Pulsed radio frequency energy (PRFE), which used as an alternative therapeutic applications in biological tissue therapies by producing smallelectrical currents on the wound healing of $\mathrm{Na}$ 2b cells.

Methods: Cells were grown in culture flasks in a humidified incubator at $37^{\circ} \mathrm{C}$ with $5 \% \mathrm{CO} 2$ and were used at the prolif- 
eration and confluent stages. In the presence of fibroblast growth factor (FGF)/ epidermal growth factor (EGF) in serum-freemedia, $\mathrm{Nb2a}$ cells were induced extent of neurite elongation and cells become neuronal cells. Wound model was performed with needle of ppdsyringe in shape of $(+)$. Cultured cells were exposed to the PEMF $(75 \mathrm{~Hz}$ frequency sinosoidal waveform) and PRFE (27.12 MHz carrier frequency square waveform) systems for 5 and 24 hours. The wound healing was investigated by closure of the wound by the cell proliferation with neurite inhibition using inverted microscope image.

Results: At the end of the experiment the control group compared with application group has not yet closed the wound in neuroblastoma cells In the neurons that have neurite inhibition is much more lower than neurite-freeneurons. This decreation and delation is significant after 5 hours compared to 24 hours. Statistical analysis was performed on scored images.

Conclusion: Electromagnetic field applications decrease the proliferations of the wounded neuroblastoma cells and on the otherhand give a proliferative sign also the neurite neurons which means accelerate the wound healing process.

Keywords: pulsed electromagnetic field, neuroblastoma cell line, cancer, culture, wound healing

\section{0-022}

\section{Epigenetic evaluation of the effects of cranial irradiation on mice brain}

$\underline{\text { Koç } T^{1}, \text { Yilmaz EB }}{ }^{2}$, Şahin $L^{3}$, Ergenoğlu $T^{3}$, Öztürk NC, Öztürk $\mathrm{H}^{1}$

'Department of Anatomy, Mersin University Medical Faculty, Mersin, Turkey; ${ }^{2}$ Department of Physiology, Mersin University Medical Faculty Research Hospital, Mersin, Turkey; ${ }^{3}$ Department of Physiology, Mersin University Medical Faculty, Mersin, Turkey

Objective: After a long period of early cranial irradiation effects on adult hippocampal neurogenesis has been studied to evaulate with epigenetic perspective.

Methods: Single dose of 8? Gray (Gy) whole cranial irradiation at postnatal day 14 (P14) (Rad+ Group) or double doses (Rad++ Group) of 8 Gy both at P14 and P21 were administered to the C57BL/6J female pups. Additionally, a group of age and body weight matched mice were assigned as anesthetic or naive controls. Seven months after the cranial irradiation, all groups were first assigned for Open Field test to measure the locomotor activity, and afterwards for Morris Water Maze paradigm to test the hippocampal dependent spatial learning and long term memory. Also, immunohistochemical stainings were employed with phenotypic neuronal and epigenetic markers.

Results: In the Morris Water Maze experiments, Rad+ and $\mathrm{Rad}++$ groups displayed significantly weaker cognitive abilities as compared to the controls. Lastly, a significant dose-dependent difference of irradiation was also detected. We found a significant decrease of Doublecortin (DCX)-im (immature neuron marker) at the inner granule cell layer of dentate gyrus of irradiated mice as compared to the controls. In the same hip- pocampal regions, there were also significant reduction of DNA methylation determinants (DNMT3a-im and MethylCpG Binding Protein 2).

Conclusion: Our overall data suggests that exposure of cranial irradiation to the young brain alters not only the neurogenesis but also the epigenetic profile in adult hippocampus which may reflect the cellular base of the weakened cognitive abilities observed in the Morris Water Maze experiments.

Keywords: adult hippocampal neurogenesis, cranial irradiation, DNA methylation, epigenetic

\section{0-023}

\section{Characterization hypothalamic-hypophysial axis injury models in rats}

Ulutabanca $\mathrm{H}^{1}$, Gergin $\mathrm{S}^{1}$, Tanrıverdi $\mathrm{F}^{2}$, Küçük A ${ }^{1}$, Yücel D ${ }^{3}$, Başaran $\mathrm{E}^{4}$, Sönmez $\mathrm{MF}^{5}$, Bilgen $\mathrm{M}^{6}$, Keleştimur $\mathrm{F}^{2}$,

Selçuklu A

'Department of Neurosurgery, Erciyes University, Kayseri, Turkey, ${ }^{2}$ Department of Endocrinology, Erciyes University, Kayseri, Turkey; ${ }^{3}$ Department of Medical Biology, Erciyes University, Kayseri, Turkey; ${ }^{4}$ Department of Physiology, Erciyes University, Kayseri, Turkey; ${ }^{5}$ Department of Histology and Embryology, Erciyes University, Kayseri, Turkey; ${ }^{6}$ Department of Biophysics, Adnan Menderes University, Aydın, Turkey

Objective: Dysfunction of hypothalamic-hypophysial axis is a common event following a traumatic brain injury. But, the current understanding of the postraumatic events is limited due to a lack of appropriate animal models. Our aim was therefore to develop injury models with rats for use in experimental reseach and demonstrate the characteric differences in the underlying pathophsiological mechanisms.

Methods: Rats (n=93, 10-12 weeks old, 280-360 g) were subjected to head injuries under general isoflurone anesthesia using three methods; Marmarau (250 g of weight was dropped from $1 \mathrm{~m}$ height on closed head), Feeney (250 $\mathrm{g}$ of weight was dropped from $1 \mathrm{~m}$ height on $3 \mathrm{~mm}$ steel disk placed on exposed brain) and Bilgen (controlled cortical impact of exposed brain with $5 \mathrm{~mm}$ injury tip, $15 \mathrm{~m} / \mathrm{s}$ velecity, $85 \mathrm{~ms}$ duration and $3 \mathrm{~mm}$ penetration depth). After 4 weeks postinjury, bloods samples were collected and the levels of LH, FSH, Testastrone, IGF1, Cortikosteron andT4 were measured. The rats were tested for behavior and the Morris water maze performance, and then sacrificed for histopathological analysis.

Results: The rats injured with Marmarau model exhibited greater anxiety and increased neuronal tissue damage and degeneration. Those in the Feeney group performed lower in swimming task, likely due to the TBH deficiency as measured. The effects in the Bilgen group were minimal.

Conclusion: In conclusion, Marmarau injury model appears to be better suited for use in experimenal studies as it closely simulates the basic clinial symtoms of hypothalamic-hypophysial axis injury in humans.

Keywords: traumatic brain injury, hypothalamic-hypophysial axis, injury model, dysfunction 


\section{0-024}

Effects physical exercise on brain structures among schizophrenia patients - an evaluation

$\underline{\text { Ercegil G }}^{1,3,4}$, Alptekin $\mathrm{K}^{2}$

${ }^{1}$ Department of Psychology, Ege University Faculty of Letters, Izmir, Turkey; ${ }^{2}$ Department of Psychiatry, Dokuz Eylul University Medical Faculty, Izmir, Turkey; ${ }^{3}$ Department of Clinical Neuroscience, Dokuz Eylul University Health Sciences Institute, Izmir, Turkey; ${ }^{4}$ Department of Psychology, Ondokuz Mayıs University Faculty of Science and Letters, Samsun, Turkey

Objective: Physical activity has been a very popular issue as a supportive part of psychiatric treatment recently. Especially its help on metabolic health problems of schizophrenia patients is known quite well. But it is still unclear how it effects in the brain level. This study evaluates findings of research on the effects of physical activity on brain structure among schizophrenia patients discussing it with the studies of healthy subjects.

Methods: Out of the search we have made through pubmed, we could find 1185 articles published until 20 March 2015. Only 5 of them were found to be looking at effects of physical activity on brain structure in which two of them were actually the same research. As a result, we ended up with 4 clinical trials.

Results: The trials have examined the effect of physical activity in hippocampal volume, gray matter density, total brain volume, and extrastriatal body area in the brain. The studies of physical acitivities regarding healthy subjects are known to have significant differences in these areas. However, there has not been a difference among schizophrenia patients in total brain volume and gray matter density but for hippocampal volume there has been different results.

Conclusion: Results of the studies still do not draw a clear line on the effects of physical exercise among schizophrenia patients. The need for more trials and comparisons with bigger samples is still required.

Keywords: schizophrenia, brain, hippocampus, physical activity, gray matter

\section{0-025}

Neuroprotection by levetiracetam against rotenoneinduced Parkinson's disease in rats

Erbaş $\mathrm{O}^{1}$, Y1lmaz $\mathrm{M}^{2}$, Taskiran ${ }^{\mathrm{D} 3}$

'Department of Physiology, Istanbul Science University School of Medicine, Istanbul, Turkey; ${ }^{2}$ Department of Neurology, Mugla Sitkı Kocman University School of Medicine, Mugla, Turkey; ${ }^{3}$ Department of Physiology, Ege University School of Medicine, Izmir, Turkey

Objective: Levetiracetam (LEV), a second-generation new anti-epileptic drug, is used for both focal and generalized epilepsy. Also, there is growing body of evidence suggesting that LEV may have neuroprotective effects. The present study was undertaken to investigate the neuroprotective effects of $\mathrm{LEV}$ on rotenone-induced Parkinson's disease $(\mathrm{PD})$ in rats.
Methods: Twenty-four adult Sprague-Dawley rats were infused with rotenone $(3 \mu \mathrm{g} / \mu \mathrm{l}$ in DMSO) or vehicle $(1 \mu \mathrm{l}$ $\mathrm{DMSO}$ ) into the left substantia nigra pars compacta $(\mathrm{SNc})$ under stereotaxic surgery, and PD model was assessed by rotational test ten days after drug infusion. The valid PD rats were randomly distributed into two groups; Group $1(n=7)$ and Group $2(\mathrm{n}=7)$ were administered saline $(1 \mathrm{ml} / \mathrm{kg} /$ day, i.p. $)$ and LEV $(600 \mathrm{mg} / \mathrm{kg} /$ day, i.p.) through 21 days, respectively. The effects of LEV treatment were evaluated by behavioral (rotation score), biochemical (oxidant/antioxidant) and immunohistochemical (tyrosine hydroxylase and caspase-3) parameters.

Results: Apomorphine-induced stereotypic rotations in PD rats were significantly suppressed by LEV treatment. While unilateral rotenone lesion induced a dramatic loss of dopaminergic neurons both in the striatum and $\mathrm{SNc}$, LEV treatment significantly attenuated the degenerative changes in dopaminergic neurons. Furthermore, LEV significantly decreased malondialdehyde levels, a marker of lipid peroxidation, and induced glutathione levels and catalase activity in PD rats compared with saline group.

Conclusion: We conclude that LEV may have beneficial effects on dopaminergic neurons against rotenone-induced injury. The underlying mechanism may be associated with the attenuation of oxidative stress and inhibition of apoptotic pathways.

Keywords: levetiracetam, Parkinson's disease, rotenone, oxidative stress, apoptosis

\section{0-026}

\section{Investigation of the relationship between Parkinson's disease and alpha-synuclein gene polymorphisms}

Kurman $\mathrm{Y}^{1}$, Gezen Ak D ${ }^{1}$, Dursun $\mathrm{E}^{1}$, Apaydın $\mathrm{H}^{2}$, Alaylıoğlu $M^{1}$, Gündüz A², Bilgiç B ${ }^{3}$, Kızıltan $\mathrm{G}^{2}$, Hanağası $\mathrm{H}^{3}$, Ertan $\mathrm{S}^{2}$, Yilmazer $\mathrm{S}^{1}$

'Department of Medical Biology, Istanbul University Cerrahpasa Faculty of Medicine, Istanbul, Turkey; '2Department of Neurology, Istanbul University Cerrahpasa Faculty of Medicine, Istanbul, Turkey; ${ }^{3}$ Department of Neurology Behavioral Neurology and Movement Disorders Unit, Istanbul University Istanbul Faculty of Medicine, Istanbul, Turkey

Objective: Parkinson's disease is a progressive neurodegenerative disease seen with movement disorder. It has been suggested in several case-control and GWA studies that a genetic variation in the SNCA gene ( $\alpha$-Synuclein) is a risk factor for developing Parkinson's disease. In our study, we aim to investigate the association between SNCA single nucleotide polymorphisms (SNPs); rs356219 and rs2736990 with Parkinson's disease in a cohort of Turkish population.

Methods: Peripheral blood samples of 257 patients with Parkinson's disease (184 late onset, 74 early onset) and 165 aged-matched healthy controls were included. SNPs in the SNCA gene were analyzed with real-time polymerase chain reaction method. 
Results: The genotype and allele distributions of both regions did not significantly differ between patients and control groups. Comparing the rs356219 as AG+GG genotypes together vs. AA genotype, we found that AG+GG genotype frequency significantly higher than AA genotype frequency in late onset Parkinson patients comparing with the healthy controls $(\mathrm{p}=0,037)$. The allele and genotype distrubitions of both regions were compared between late-onset and early-onset Parkinson patients also. AG genotype frequency of rs356219 was found to be higher in late onset PD cases and GG genotype frequency was found to be higher in early-onset patient group, yet the statistical difference was nearly significantly $(\mathrm{p}=0.087)$.

Conclusion: Our study showed that SNCA rs356219 single nucleotide polymorphism is associated with increased risk of Parkinson's disease.

Keywords: Parkinson's disease, SNCA ( $\alpha$-Synuclein) gene, single nucleotide polymorphism

\section{0-027}

Effects of the propolis on spatial memory induced by lithium-pilocarpine in the SE model in rats

Emre MH, Kaya GB

Department of Physiology, Inonu University Faculty of Medicine, Malatya, Turkey
Objective: In our study, protective and therapeutic effects of propolis were investigated in lithium-pilocarpine-induced rats with SE. Propolis effects on spatial memory were examined on the Morris Water Maze.

Methods: 50 Sprague-Dawley female rats were divided into five groups $(\mathrm{n}=10)$. Group 1 (control), group 2 (group treated with propolis before the experiment by oral gavage+Li-Pc intraperitonally), group 3 (Li-Pc+diazepam+propolis), group 4 (Li-Pc+propolis) and group 5 (only Li-Pi i.p; epileptic group). Propolis effects on spatial memory were examined on the Morris Water Maze.

Results: According to the results obtained from the spatial memory in the Morris water maze, while the lenght of stay in the dial in propolis-treated group (Group 3 and 4), when compared with epileptic group (group 5), increased, the delay time and distances to the platform decreased statistically significant $(\mathrm{p}<0.05)$.

Conclusion: Our results reveal that propolis has a characteristic which can repair the neurological damage that SE create in children's spatial memory and that this substance could be used as a preventive and anti-epileptic agent in the treatment of SE.

Keywords: epilepsy, propolis, status epilepticus, spatial memory, rat 


\title{
Poster Presentations
}

\author{
(P-001 - P-109)
}

\section{P-001}

Effects of maternal deprivation and enriched environment on NMDAR level and memory in adult mice

$\underline{\text { Akıllığlu K}}{ }^{1}$, Y1lmaz $\mathrm{MB}^{2}$, Boğa A ${ }^{1}$, Binokay $\mathrm{S}^{1}$, Kocatürk-Sel S

'Department of Medical Physiology, Cukurova University Faculty of Medicine, Adana, Turkey; ${ }^{2}$ Department of Medical Biology, Cukurova University Faculty of Medicine, Adana, Turkey

Objective: Early adverse life experiences have been associated with learning-memory impairment. N-methyl-D-aspartate receptors (NMDARs) play an important role in brain developmental processes. Enriched environments are known to positively influence cognitive functions in brain. In our study was examined the effects of MD on NMDA receptor subunits in the hippocampus and learning-memory performance of Balb/c mice and whether these could be reversed by later raising the offspring in an enriched environment.

Methods: For this purpose, mice were separated from their mothers for a 24-h single episode at postnatal day (PND) 9. They were weaned on day 21 and were housed under either standard (SE) or enriched (EE) environmental conditions. Cognitive processes of mice were evaluated using a Morris water-maze (MWM). In the hippocampus, NMDAR subunits (NR1, NR2A and NR2B) mRNA expression levels were examined by real-time PCR.

Results: MD decreased spatial learning-memory performance in the MWM $(\mathrm{p}<0.05)$, however this effect did not reverse by $\mathrm{EE}(\mathrm{p}<0.001) . \mathrm{MD}$ (in the SE and EE conditions) increased NR1, NR2A $(\mathrm{p}<0.001)$ and NR2B $(\mathrm{p}<0.05)$ mRNA expressions in the hippocampus.

Conclusion: In conclusion, MD led to deterioration of cognitive processes during adulthood. Moreover, enriched environment did not reverse the deleterious effect of the MD on cognitive functions and increased NMDAR levels. Funding for this study was provided by the Scientific Research Office of Cukurova University (I.U BAP, Project no: TF2011BAP31).

Keywords: maternal deprivation, environmental enrichment, hippocampus, NMDAR

\section{P-002}

Comparing the facial emotion recognition ability between interns who prefer departments of internal medicine or surgery

Cetinkaya S, Altun I, Uludag C, Ozan E, Gultekin G, Yuksek E, Emul M

Department of Mental Health and Diseases, Istanbul University, Cerrahpasa Medical Faculty, Istanbul, Turkey
Objective: Recognizing facial expressions, including less obvious facial micro-expressions of patients may be useful for the interaction between doctor and patients (Ekman 2004). Thus, we hypothesized that medical students who prefer surgical departments might have less ability to recognize facial emotions and might need more time to response each facial emotions than medical students who prefer different internal medicine departments.

Methods: Anonymously, 101 medical students were enrolled in the study. The preferences of medical students' were divided into two according to their preference of surgery or internal medicine departments. Then, a manual-based test included the photos of four male and four female models (totally 56 mixed photos supported as packaged for software by Ekman \& Friesen) with happy, surprised, fearful, sad, angry, disgusted, and neutral facial expressions from Ekman \& Friesen's series. Student's $t$ and Mann Whitney U Tests were used to compare mean accuracy rates of FER between groups.

Results: There were 59 medical students who preferred internal medicine departments while the rest $(n=42)$ preferred surgery departments. The mean accuracy rates of FER ability between two groups were insignificant: for happy $(6.93 \pm 0.314$ vs $6.98 \pm 0.154, \mathrm{p}=0.355)$; for angry $(5.20 \pm 0.846$ vs $5.10 \pm 0.790$, $\mathrm{p}=0.512)$; for surprised $(5.31 \pm 0.951$ vs $5.24 \pm 0.821, \mathrm{p}=0.706)$; for $\operatorname{sad}(2.47 \pm 1.131$ vs $3.82 \pm 6.771, \mathrm{p}=0.654)$, for fear $(4.41 \pm 1.984$ vs $4.48 \pm 1.366, \mathrm{p}=0.730)$; for disgust $(4.07 \pm 1.856$ vs $4.40 \pm 1.668, \mathrm{p}=0.930)$; for neutral $(6.64 \pm 0.609$ vs $6.57 \pm 0.737$, $\mathrm{p}=0.602)$. There were no significant differences according to requested time to recognize each facial emotion between groups $(\mathrm{p}>0.05)$.

Conclusion: This study is the first which compared the ability of FER between medical students who prefer surgery and internal medicine. Our study is a preliminary study including relatively small sample size. There might be some other factors which may be related with poor patient doctor relationship than FER ability.

Keywords: facial emotion recognition, surgery, internal medicine, medical students

\section{P-003}

Cognitive skills and archery performance of the children at the ages of 10-11

$\underline{\text { Dal N}^{1}}$, Kutlu N ${ }^{2}$, Çobanoğlu $\mathrm{G}^{1}$

'Physical Education and Sport Teaching, Celal Bayar Üniversitesy, School of Physical Education aad Sport, Manisa, Turkey; ${ }^{2}$ Department of Physiology, Neurophysiology Sub-Department, Celal Bayar University, Yunus Emre, Manisa, Turkey 
Objective: The purpose of the study is to observe the effect of psychomotor skills such as coordination, comprehension ability, attention and reaction on the Archery performance points.

Methods: The study group was composed of children at the ages of 10-11, 44 of them being the subject group and 30 the control. Archery Education was provided for 12 weeks, twice a week and 1.5 hour sessions and performance points were measured. Pre and post Archery Education cognitive skills such as comprehension (spm), attention (cog) and reaction time and quality (dt) were measured by Vienna Testing System.

Results: In terms of cognitive skills such as comprehension (spm), attention (cog) and reaction time and quality (dt) significant statistical differences were found in the Pre and post Archery Education measurements of the subjects $(\mathrm{p}<0.05)$. When the correlation between archery points and other variables is measured, a positive correlation $(p<0.01)$ is found between the comprehension ability and the archery points.

Conclusion: the results of the study shows that the children at the ages of 10-11 who received archery education improved in comprehension (spm), attention ( $\operatorname{cog}$ ) and reaction time and quality (dt)skills than the ones who did not. It can also be mentioned that archery motor skills and cognitive skills have a significant effect on each other.

Keywords: archery performance, Vienna test system, cognitive function

\section{P-004}

The relationship between the cognitive skills of the right and left-handed children and archery performance

$\underline{\text { Kutlu N}}{ }^{1}$, Dal N², Çobanoğlu G ${ }^{2}$

'Department of Physiology, Neurophysiology Sub-Department, Celal Bayar Üniversitesy, Yunus Emre, Manisa, Turkey; ${ }^{2}$ Physical Education and Sport Teaching, Celal Bayar University, School of Physical Education and Sport, Manisa, Turkey

Objective: The purpose of the study is to compare the dominant hand choices with archery performance and cognitive and psychomotor skills such as coordination, comprehension ability, attention and reaction time of the volunteers.

Methods: The study is composed of a volunteer group of 20 right handed and 15 left handed children. The choice of hand is determined by Edinburg Survey. Archery Education to the volunteers was provided for 12 weeks, twice a week and 1.5 hour sessions and performance points were measured. Pre and post Archery Education cognitive skills such as comprehension (spm), attention (cog) and reaction time and quality (dt) were measured by Vienna Testing System.

Results: Children with +80 hand choice range lateralization coefficient were determined as right-handed while the ones with -70 were left-handed. Left-handed ones were more successful in Archery than Right-handed ones. A positive correlation was observed between archery points and spm which is one of the cognitive skills $(\mathrm{p}<0.01)$.
Conclusion: The study compared the dominant hand choices with archery performance and cognitive skills. The conclusion of the dominant hand choices, archery performance and cognitive skills affecting each other significantly was reached. Lefthanded children's success compared to the right handed ones was observed. It can also be mentioned that motor skills, cognitive functioning and lateralization coefficient hand a significant relation.

Keywords: archery, hand preference, psychotechnic, cognitive fonction, performance

\section{P-005}

The effects use monosodium L-glutamate in obesity, behavior and memory in male rats

Yazgan K ${ }^{1}$, Gölgeli A ${ }^{1}$, Özyürek Ö$^{2}$, Çalışkan F

'Department of Physiology, Erciyes University, Kayseri, Turkey, ${ }^{2}$ Department of Exercise Physiology, Erciyes University, Kayseri, Turkey

Objective: Monosodium glutamate (MSG) is sodium salt of glutamic acid and to flavor foods used often a food additive. Susceptible individuals in MSG consumption is known that severe chest tightness, pressure sensation, headache, heart attack, lead obesity, fatty liver. This study, male rats in the long-term use monosodium glutamate aimed to investigate effects of obesity, memory behavior.

Methods: 4 month Wistar albino rats were used in this study. The control, MSG were formed each consisting of 10 groups. MSG is added to drinking water provided take for 12 weeks. MSG calculated joined 1.5 liters drinking water $250 \mathrm{mg} / \mathrm{kg}$ will have based total weight animals in cage. The open field were evaluated pass line, freezing, itching, defecation. Three panel were tested reference and working memory. Experimental group were calculated body weights, feed amount, amount of water consumed.

Results: MSG treated groups compared control group shows the number of line crossings in open area, devices hindlimbs rise behavior $(\mathrm{p}<0.05)$ was more seen significantly changed locomotor activity. Three panel in reference memory, working memory, eating the time of arrival to the number of errors; the groups were observed significant differences between for three days $(\mathrm{p}<0.05)$. MSG group was observed significant decrease statistically $(\mathrm{p}<0.05)$.

Conclusion: Wistar male rats applications MSG did not cause obesity for 12 weeks, open field apparatus did not change increased locomotor activity, the autonomic function. MSG applications has emerged negative effect on memory were evaluated the test parameters three panels. This work was supported project by Erciyes University Research Fund No. TYL2014-5462.

Keywords: monosodium glutamate, three-panel test, open field, behavior, rat 


\section{P-007}

Evaluation memory with three panel test generated high calorie and hunger model in rats

Yazgan K, Keklik E, Başaran KE, Gölgeli A

Department of Physiology, Erciyes University, Kayseri, Turkey

Objective: When high calorie foods fed more the body needs occurs increase in adipose tissue also it has affected limbic structures it can be observed change in behaviour, emotional and cognitive functions. This study; high in calories, hunger model in rats by creating aimed to evaluate memory with three panel test.

Methods: 36 males (Wistar albino) rats were used this study. Control, fasting, high calorie groups were formed each including twelve rats. Hunger group of rats starved for 96 hours were allowed.High calorie grub were fed with fat diet adding 25 grams butter 100 grams of pellets for 12 weeks. In open field assembly were evaluated ocomotor activity, autonomic functions, three panels in testing apparatus memory for three days.

Results: Experimental groups were taken in the open field, number of line crossings, control, high-calorie, hunger groups, was found respectively $(33.75 \pm 7.2,41.83 \pm 5.68,38.16 \pm 6.75)$. Locomotor activity fasting group is much more significant than control group $(\mathrm{p}<0.05)$. The number of hindlimbs increase was found in control, hunger and high calorie grubs respectivly $(\mathrm{p}<0.05)$. Three panel test in the time reach eating at memory; was significanted differences $(\mathrm{p}<0.05)$.

Conclusion: The increase in locomotor activity and exploratory behavior in fasting rats shows that the directed to food-seeking behavior. Fat diet reduced the exploratory behavior. Three panels test was affected of reference and working memory. This work was supported by TUBITAK project No. 114S009.

Keywords: reference memory, working memory, high-calorie, starvation, open space

\section{P-008}

Investigation of the effects of learning swimming exercise after calorie restriction in obese rats

Yazgan K, Erten NB, Gölgeli A

Department of Physiology, Erciyes University, Kayseri, Turkey

Objective: Excessive weight gain and obesity is a factor for diabetes, hyperlipidemia, hypertension cardio vascular diseases. This study, obesity rats post calorie restriction lnvestigation effect of swimming exercise learning.

Methods: Three month female Sprague-Dawley rats were used in this study.Control, obese, obese + swimming + swimming + calorie restriction group were created including three groups of 10 animals. High calorie grub, were fed with fat diet adding 25 grams butter 100 grams pellets for 8 weeks. Obese+ swimming, obese + swimming + to calorie restriction group was subjected to swimming exercise $(60 \times 25 \times 40) 10$ days of continuous swimming in tank for 15 minutes.Behavioral parameters was assessed spatial learning in the open space and swimming in $\mathrm{t}$ Morris test.

Results: Obesity model to the control, obese+ swimming and obese + swimming + calorie restriction group was found significantly in the number of line crossings $(45.4 \pm 7.82,46.3 \pm 4.98$ ve $22.12 \pm 4.48)$ rise in the number of respectively $(\mathrm{p}<0.05)$.In the swim test obese+ swimming + caloric restriction in group is phase significantlytime to reach the platform in the Morris teste $(\mathrm{p}<0.05)$. Their distance and speed were observed differences significant $(\mathrm{p}>0.05)$.

Conclusion: Obesity created in female rats is affected locomotor activity after 15 minutes for 10 days swimming exercis by caloric restriction.Spatial learning performance increased with caloric restriction.

This work was supported project by Erciyes University Research Fund No. TYL-2013-4291.

Keywords: obesity, fat diet, calorie restriction, swimming exercise, open space

\section{P-009}

\section{Anxiety-like behaviors in the neonatal ketamine treatment in C57BL/6 and BALB/c mice}

Akıllığlu K ${ }^{1}$, Koçaklı ZG ${ }^{1}$, Boğa A ${ }^{1}$, Binokay $S^{1}$, Karadepe $\mathrm{M}^{2}$, Akın $\mathrm{H}^{3}$, Barç $\mathrm{D}^{4}$

'Department of Medical Physiology, Cukurova University Faculty of Medical, Adana, Turkey; 24th Years Student, Cukurova University Faculty of Medical, Adana; ${ }^{3} 3$ rd Years Student, Cukurova University Faculty of Medical, Adana; ${ }^{4}$ Department of Medical Biology, Cukurova University Faculty of Medical, Adana, Turkey

Objective: Ketamine application causes behavioural and neurochemical effects in humans and experimental animals. During the critical period for brain development, a series of rapid and radical changes occur. This study, we evaluated effects of neonatal ketamine application on anxiety-like behaviors of adult Balb/c and C57BL/6 mice.

Methods: Male mice pups were injected intraperitoneally with ketamine (10 ve $20 \mathrm{mg} / \mathrm{kg}$ and a volume of $0.1 \mathrm{ml} / 10 \mathrm{~g}$ body weight) on postnatal day 7 . The same volume of saline $(0.9 \%$ $\mathrm{NaCl})$ was injected into the control group. The open-field (OF) and elevated plus maze (EPM) tests were used to evaluate anxiety-like behaviors in adulthood.

Results: In the OF and EPM test, anxiety-like behaviors were lower in C57BL/6 mice compared to Balb/c mice ( $\mathrm{p}<0.05)$.

There was no difference in the OF and EPM test receiving 10 $\mathrm{mg} / \mathrm{kg}$ ketamine in the Balb/c and C57BL/6 mice. Ketamine treatment of C57BL/6 mice at $20 \mathrm{mg} / \mathrm{kg}$ dose caused an increase in anxiety-like behavior compared to control group in the OF and EPM $(\mathrm{p}<0.05)$. In Balb/c mice, $20 \mathrm{mg} / \mathrm{kg}$ dose of ketamine treatment caused no difference in the OF and EPM test compared to control group.

Conclusion: In our study showed a decrease in anxiety-like behavior in C57BL/6 compared to Balb/c. Besides, hereditary 
factors may play an important role in the effect on the anxietylike behavior of neonatal period ketamine treatment. Funding for this study was provided by the Scientific Research Office of Cukurova University (project no: TF2013BAP22).

Keywords: BALB/c mouse, C57BL/6 mouse, ketamine, neonatal

\section{P-010}

The effects of time-restricted feeding on the blood biochemistry and behavior in rats

Doğru Jakubowska E, Gönülkırmaz Ö

Department of Biological Sciences, Middle East Technical University, Ankara, Turkey

Objective: Today, obesity is one of the most important health problems. For long, obesity has been linked to the diet type and daily caloric intake. The effect of time restricted feeding (TRF) on the weight management is less documented. The aim of this study was to investigate whether long-term night-time restricted feeding affects body weight gain, metabolic parameters, blood leptin levels, and behavioral performance in elderly overweight male Wistar rats.

Methods: For 3 months (10 years in human), 14-months old, overweight male Wistar rats $(n=6)$ were subjected to a timerestricted diet (TRF group) with food (standard lab chow) provided during $8 \mathrm{~h}$ of the dark phase of the diurnal cycle. Control group $(n=6)$, was maintained on ad libitum diet (ADLIB group). Blood levels of triglycerides, HDL, LDL and leptin were measured and a battery of behavioral tests was performed before and after the diet implementation.

Results: Longitudinal (pre-post treatment) and cross-sectional (TRF versus ADLIB diet) comparisons revealed: no between-group differences in food intake and body weight gain, locomotor activity (Open Field), anxiety levels (OF \& Elevated Plus Maze), and short- or long-term memory retention (Water Maze); positive effect of TRF on motor performance in Accelerod task; trend towards slower place learning in TRF group during post-treatment test; increase in the blood LDL and leptin levels in ADLIB but not TRF group.

Conclusion: In conclusion, prolonged time-restriction feeding even when overlapping with diurnal phase of increased activity does not significantly affect metabolic and behavioral parameters in elderly overweight male rats.

Keywords: time-restricted feeding, rat, blood biochemistry, behavior

\section{P-011}

Physiological meaning of psychotechnical evaluation of right-handed and left-handed individuals

Ölçgen B, Uyan M, Gürçinar İH, Alpay Ş, Kazdağlı H, Kutlu N

Department of Physiology, Celal Bayar University Faculty of Medicine, Manisa, Turkey
Objective: Psychotechnique evaluation to understand left or right handed individuals are whether suitable for a particular job, analyzing an individuals personality, mental psychomotor and cognitive skills and standardizing the results numerically.

Methods: Hand preference was obtainded with "Hand Preference Questionnaire”. DT, COG and SPM Tests and were applied to 100 volunteers Between the ages of 18-25 and 26-50, male-female, Intermediate-High School-Univercity or higher education level students with International computer based Vienna Test System. Datas were evaluated with the spss 15.0 programme.

Results: $18-25$ years olds group were better than 26-50 years olds in correct responses. 18-25 years olds group were better than 26-50 years olds in response time. Also reasoning (IQ) results of 18-25 years olds were better significantly than 26-50 years olds. Reasoning results of females were significantly better than males Reasoning (IQ) results of left-handed were significantly better than right-handed. Reasoning (IQ) results of Univercity or higher education level graduates were significantly better than intermediate school graduates.

Conclusion: Cognitive skill, speed and correct responses that brain's higher functions were better significantly with regard to age, gender and education.

Keywords: hand preference, gender, cognitive skill, vienna test system

\section{P-012}

\section{Comparison of curcumin administration and surgical treatment on model of neuropathic pain in rat}

Ceyhan $\mathrm{D}^{1}$, Koçman $\mathrm{AE}^{2}$, Yıldırım E ${ }^{3}$, $\underline{\operatorname{Ayd} ı n} \mathrm{~S}^{3}$, Özatik $\mathrm{O}^{4}$, Köse $\mathrm{A}^{2}$

'Department of Anesthesiology and Resuscitation/ Algology, Eskisehir University Faculty of Medicine, Eskişehir, Turkey; ${ }^{2}$ Department of Plastic Reconstructive and Aesthetic Surgery, Eskisehir University Faculty of Medicine, Eskişehir, Turkey; ${ }^{3}$ Department of Pharmacology, Eskisehir University Faculty of Medicine, Eskişehir; ${ }^{4}$ Department of Obstetrics and Gynecology / Reproductive Health Center, Eskisehir University Faculty of Medicine, Eskişehir, Turkey

Objective: Curcumin (C) was investigated in various neuropathic pain (NP) models. In experimental studies curcumin is usually applied immediately after the chronic constriction injury (CCI). However, in clinical practice patients are received curcumin after the occurrence of NP. In our study we compared the efficacy of medical treatment and the surgical treatment.

Methods: Sprague-Dawley rats were divided into 8groups: Sham, Control (CCI), CCI+Chronic-constriction-release (CCR), CCI+C, CCI+Tramadol (T), CCI+C+T, CCI+CCR+ $\mathrm{C}, \mathrm{CCI}+\mathrm{CCR}+\mathrm{T}$. The symptoms of NP were observed for 7 days. On the 7 th day, the nerve ligation was released by secondar-surgery (CCR) in CCI+CCR, CCI+ CCR+T, CCI+ $\mathrm{CCR}+\mathrm{C}$ groups. C (100 mg/kg/po) and T $(10 \mathrm{mg} / \mathrm{kg} / \mathrm{ip})$ were 
administered for 14 days after the 7 th day. During this time, mechanical allodynia (MA), thermal hyperalgesia (TH) and cold allodynia (CA) tests were performed on $0,1,3,7,13,17,21$ th days. Two-way-ANOVA and the Tukey test were used for statistical analysis.

Results: In MA, paw withdrawal threshold (PWT) was significantly different in CCI+CCR+T compared to control on the 13 th $\left(\mathrm{p}<0.05^{*}\right), 17$ th $\left(\mathrm{p}<0.05^{*}\right), 21$ th $\left(\mathrm{p}<0.01^{* *}\right)$ days. In the $\mathrm{CCI}+\mathrm{T}$ group, PWT was significantly different on the 21 th day $\left(\mathrm{p}<0.001^{* * *}\right)$ compared to control. In TH, paw withdrawal latency (PWL) in $\mathrm{CCI}+\mathrm{C}+\mathrm{T}$ group was significantly different on the 13th $\left(\mathrm{p}<0.05^{*}\right), 17$ th $\left(\mathrm{p}<0.001^{* * *}\right), 21$ th $\left(\mathrm{p}<0.001^{* * *}\right)$ days compared to control. In CCI $\mathrm{T}\left(\mathrm{p}<0.001^{* * *}\right)$ and $\mathrm{CCI}+\mathrm{CCR}+\mathrm{T}$ groups PWL was significantly different on the 21th day compared to control.

Conclusion: The efficacy of curcumin and chronic-constriction-release was not significantly effective in the reduction of NP. Tramadol alone and in combination with chronic-constriction-release was found to be effective as expected.NP was found to be decreased in curcumin and tramadol combination group $(\mathrm{CCI}+\mathrm{C}+\mathrm{T})$. Further investigations are needed with different doses and long term treatments in order to determine the alleviating effects of curcumin.

Keywords: neuropathic pain, curcumin, tramadol, chronic constriction injury, chronic constriction release

\section{P-014}

3D modeling and animation of the structural and functional properties of the human auditory system

Evren $\mathrm{M}^{1}$, Evren $\mathrm{V}^{2}$, Koylu $\mathrm{OE}^{2}$

'Department of Biotechnology, Ege University Natural and Applied Sciences, Izmir, Turkey; ${ }^{2}$ Department of Physiology, Ege University Medical Faculty, Izmir, Turkey

Objective: The usage of three-dimensional applications in education and research has been increasing in recent years. Three-dimensional models were started to employ instead of two-dimensional illustrations in many life sciences related areas such as medicine and biology. Sensory systems are the most complex ones among the neuroscience themes in terms of interpretation of their functional mechanisms. Accurate modeling of related anatomical parts and simulation of mechanisms of the sensory systems in human will provide an important contribution to the neuroscience education. Auditory system has been selected in our study. The objective of the study is to simulate the external, middle and internal ear in computer to be used for educational and research purposes.

Methods: Existing visual material in atlas, books and radiological images were screened and modeled in computer. Measures and aspect ratios of the structures were preserved.

Results: Photographs of the rendered three-dimensional models which obtained at this stage of the study presented in our poster. These virtual models were animated in computer and functional properties are shown with the moving models.

Conclusion: The difficulty of presenting three-dimensional structures in two dimensional visual materials is undisputable. In this context the major advantage of audio-visual contents is to be able to present the $3 \mathrm{D}$ structures to the audience using every angle thus providing a clear and quick perception of structural and functional properties of the topic. Additionally, the possibility of sharing a content by means of digital network gives the chance of reaching out more people in a short time.

Keywords: neuroscience education, human auditory system, 3D modeling \& animation, simulation, medical illustration

\section{P-015}

\section{Goal directed and goal free decision making mechanisms across lifespan}

Taybaş $C^{1}$, Li SC $^{2}$, Eppinger B ${ }^{2}$

${ }^{1}$ Izmir Institute of Technology, Department of Science, Molecular Biology and Genetics, Izmir, Turkey; 2Dresden University of Technology, Psychology Department, Dresden, Germany

Objective: We studied individual differences in goal-directed/free decision-making processes related to age. We focused on whether age affects decision-making, if so, how it affects in brain. While studying individual decision-making mechanisms at various age groups we used computational modelling, statistical analysis and Markov decision-making test.

Methods: In Markov test containing two stages, participants must update their predictions according to reward. Participants composed of 56 young individuals $(20-30$ years) and 60 older adults (56-78 years) must choose a choice presented. Choice in first stage determines transition probability of the one of choices presented to participant in second stage. Transitions are common (70\%) and rare (30\%). Choice in first stage will determine choice type (common or rare transition) in second stage. Now, participants must choose one of two cartoon characters and will gain reward if they choose right one. Probability of characters to be shown is determined by an algorithm randomly. Actual purpose of test is to determine if participants change their decisions following characters they gain reward. Also test aims determining whether participants find right character to gain reward and the time intervals these processes occur.

Results: It is shown young individuals could solve general structure of test and change their decisions according to it. However, old individuals seem persisting in their decisions along test and have difficult to solve structure of Markon decision-making test. Working memory (WM) is an important indicator for goal-directed and goal free mechanisms in participants.

Conclusion: We found young individuals exhibit high WM performance in exploring tasks and goal-directed decisionmaking effectively. However, it's discovered there is no rela- 
tionship between WM and decision-making mechanisms in old individuals.

Keywords: decision-making, working memory, markov test

\section{P-016}

The relationship between synaptic proteins and brain aging in male-female zebrafish (Danio rerio)

Karoğlu ET $^{1}$, Erkaya B ${ }^{2}$, Arslan-Ergül A3 , Altaytaş F Halim DÖ ${ }^{2}$, Konu Ö$^{4}$, Adams $\mathrm{M}^{5}$

${ }^{1}$ Interdiciplinary Neuroscience Program, Bilkent University Graduate School of Engineering and Science, Ankara, Turkey; ${ }^{2}$ Department of Molecular Biology and Genetics, Bilkent University, Faculty of Science, Ankara, Turkey; ${ }^{3}$ Department of Molecular Biology and Genetics, Molecular Biology and Genetics Zebrafish Facility, Bilkent University, Faculty of Science, Ankara, Turkey; ${ }^{4}$ nterdisciplinary Program in Neuroscience, Department of Molecular Biology and Genetics, Zebrafish Facility / Graduate School of Engineering and Science, Faculty of Science, Bilkent University, Ankara, Turkey; Interdiciplinary Neuroscience Program, Department of Psychology, Molecular Biology and Genetics Zebrafish Facility / Graduate School of Engineering and Science, Bilkent University, Faculty of Economics, Administrative and Social, Sciences, Bilkent University, Ankara, Turkey

Objective: Determining whether the adult zebrafish brain ages in a sexually dimorphic manner has not been well-established yet and studies are just beginning to examine this question. The aim of the current study was to reveal the pattern of agerelated changes of three key synaptic proteins associated with excitatory-inhibitory synapses, pre-synaptic vesicle protein; synaptophysin (SYN), post-synaptic density-95 (PSD95) protein, and gephyrin (GEP) protein. Based on brain aging studies in other species, we hypothesized; these levels would decline and the pattern of aging would be different in male and female zebrafish.

Methods: Whole brain tissues were isolated from young (6-8 months), middle-aged (12-14 months) and old (27-30 months) male and female zebrafish (AB strain). Animals were maintained and raised in standard conditions in the zebrafish facility. The extracted brain tissue was homogenized in RIPA buffer and subjected to Western Blot analysis to determine differences in the relative protein expression levels.

Results: Our results demonstrated that there was a significant decline in SYN protein levels with age, as well as a significant age by gender interaction; females tend to preserve SYP levels in old age, whereas in males SYP levels are reduced in old age. For GEP, we observed a significant main effect of gender with females having more GEP than males. Finally, for PSD-95, there was a significant age-gender interaction; in males PSD95 levels decrease with age but in females PSD-95 levels are preserved in old age.

Conclusion: Our data suggest that selected synaptic protein levels decline with age and might be differentially expressed according to gender.

Keywords: aging, gender, synaptic proteins

\section{P-017}

Fundamental approaches to the measurement of volume of brain regions from magnetic resonance images

Sağsöz ME ${ }^{1}$, Sade $\mathrm{R}^{2}$, Oral E ${ }^{3}$, Oral E ${ }^{4}$

'Department of Biophysicst, Ataturk University Faculty of Medicine, Erzurum, Turkey; ${ }^{2}$ Department of Radiology, Ataturk University Faculty of Medicine, Erzurum, Turkey; ${ }^{3}$ Department of Psychiatry, Ataturk University Faculty of Medicine, Erzurum, Turkey; ${ }^{4}$ Department of Psychiatry, Ataturk University Faculty of Medicine, Erzurum, Turkey

Objective: In this study, our aim was to compare the volume of some brain regions determined by automatic segmentation software and manually by two experts over the magnetic resonance images.

Methods: Volumes of some brain areas are compared which are calculated with FreeSurfer (Martinos Center for Biomedical Imaging, Laboratory for Computational Neuroimaging in Charlestown, MA, USA) an open source segmentation software and calculated by two specialists manually on same images obtained with different $\mathrm{T} 1$ and $\mathrm{T} 2$-weighted magnetic resonance sequences.

Results: Volumes obtained by automatic and manual evaluations was shown to be similar $(\mathrm{p}<0.05)$.

Conclusion: Automatic segmentation softwares that are based on digital atlases and contour determination algorithms can be used safely in the calculation of volume of different brain areas on magnetic resonance images.

Keywords: magnetic resonance, segmentation, brain

\section{P-018}

The frequency information of striatal synchronisation investigated with computational model

Elibol R, Şengör NS

Electronics and Communication Engineering, Istanbul Technical University, Faculty of Electrical and Electronic Engineering, Istanbul, Turkey

Objective: The basis of signals observed in EEG data and known to be essential in various cognitive processes and mental illness is the spiking of neuron groups in harmony. In this work, the aim is to determine the frequency of synchronous behavior with time-frequency analysis of the computational model and to observe the role of modification of synaptic weights on frequency.

Methods: A computational model of dorsal striatum has been proposed and using this model simulations are carried out in BRIAN environment. In the simulations, the role of neurotransmitter Dopamine modulating the effect of inputs from cortex to striatal neurons on synchronization has been observed. The effect of Dopamine has been modeled according to biological plausibility by changing the weights of synaptic connections. 
Results: The relation between Dopamine and the synchronous behavior in dorsal striatum has been revealed. The activation and synchronization measures of neurons with D1 and D2 type receptors are changing according to Dopamine levels. While increasing the Dopamine level enhances the activity and synchronization measures of neurons with D1 type receptors, the activation and synchronization of neurons with D2 type receptors are decreased. Reducing the Dopamine level has just opposite effect on neurons with both type of receptors.

Conclusion: The connection dynamics of excitatory postsynaptic neuron (EPSP) and the local field potential (LFP) of local group of neurons have similar behavior. The LFP do change with Dopamine level and it carries information about the frequency of synchronizations while the Dopamine level varies.

Keywords: dopamine, striatum, basal ganglia, synchronization

\section{P-019}

\section{Mast cell degranulation induces nociceptive firing via serotonin in rat meninges}

$\underline{\text { Kulınç E }}$, Töre F $^{2}$, Giniatullin $\mathrm{R}^{3}$

'Department of Physiology, Abant Izzet Baysal University Faculty of Medicine, Bolu, Turkey; '2Department of Physiology, Sanko University Faculty of Medicine, Gaziantep, Turkey; ${ }^{3}$ Department of Neurobiology, Eastern Finland University Institute of A. I. Virtanen for Molecular Sciences, Kuopio, Finland

Objective: Pro-inflammatory molecules released from degranulated mast cells likely contribute to migraine attack by stimulating meningeal nociceptors. However, the role of serotonin, also known to be released from mast cells on activity of meningeal nerve terminals was not directly tested so far. Therefore, in the current project we aimed to investigate the action of endogenous serotonin released during degranulation of mast cells on nociceptive firing in rat meninges.

Methods: Isolated skulls obtained from adult Wistar rats were divided into 2 halves ( $\mathrm{n}=18$, totally 36 hemiskulls, 130-170 g). Using suction electrode, nociceptive firing in trigeminal nerve was recorded. Compound 48/80 (10 $\mu \mathrm{g} / \mathrm{ml}$, mast cell degranulating agent) was applied alone or with the broad spectrum antagonist of ATP-activated P2 receptors suramin $(100 \mu \mathrm{M})$, the blocker of TRPV1 receptors capsazepine $(50 \mu \mathrm{M})$, the blocker of TRPA1 receptors HC-030031 $(10 \mu \mathrm{M})$, and the 5HT3 antagonist MDL-72222 (30 $\mu M)$. Data were analyzed using Origin 8.5 software.

Results: The compound 48/80 promoted massive degranulation of mast cells in isolated meningeal preparation and induced strong nociceptive firing in trigeminal nerve endings $(\mathrm{p}<0.05)$. Out of all agents tested only MDL-72222 prevented the nociceptive firing induced by the compound $48 / 80$. Notably, the firing was completely blocked by TTX $(1 \mu \mathrm{M}$, sodium channel blocker).

Conclusion: Nociceptive firing induced by the compound $48 / 80$ was blocked by the antagonist of 5-HT3 receptors suggesting that serotonin is the active endogenous substance released from mast cell granules to activate local meningeal nerves. Our data are consistent with view that meningeal mast cells contribute to headaches primarily via serotoninergic mechanisms.

Keywords: mast cell, serotonin, nociceptive firing, migraine pathophysiology

\section{P-020}

\section{Potential role of Elk-1 transcription factor in mitosis}

$\underline{\mathrm{Uyar}}_{\mathrm{AO}}{ }^{1}$, Demir $\mathrm{O}^{2}, \mathrm{Y}_{1} 1 \mathrm{maz} \mathrm{B}^{3}$, Kurnaz Aksan I

${ }^{\prime} Y$ editepe University, Faculty of Engineering, Department of Genetics and Bioengineering, Istanbul, Turkey; ${ }^{2}$ Max Planck Institute of Molecular Cell Biology and Genetics, Howard Lab, Dresden; ${ }^{3}$ Yeditepe University Faculty of Medicine, Department of Physiology, Istanbul, Turkey; ${ }^{4}$ Gebze Teknik University, Department of Molecular Biology and Genetics, Kocaeli, Turkey

Objective: Elk-1 is a member of the ternary complex factor (TCF) transcription factors that involved in many biological processes. It was demonstrated by our laboratory that, Elk-1 transcription factor translocate different localizations during mitosis and it interacts with motor proteins, dynein and kinesin. In this study, it is intended to identify the interaction between Elk-1 and mitotic kinases, such as mitotic Polo-like kinase 1 (Plk-1) kinase, to identify the potential role of Elk-1 during mitosis.

Methods: To reveal the potential role of Elk-1 during mitosis, dot blot, immunoprecipitation, GST-Pull down and immunofluorescence assays were used. Elk-1 protein was precipitated with Elk-1 specific antibody analyzed by Dot and Western blot assays. To analyze the exact binding site on Elk-1 protein domain GST-Pull down e was used. To examine the localization of phospho-Elk-1 during different stages of cell cycle, immunofluorescence analysis was used.

Results: Preliminary immunoprecipitation (IP) and dot blot experiments indicated a strong interaction between Elk-1 and mitotic Polo-like kinase 1 (Plk-1). This interaction is prominent during the first 30 minutes of mitosis and interestingly, disappeared between 30th and 60th minutes which again observed at 90th minutes. GST Pull-down demonstrated that Plk1 kinase binds N-terminal half of Elk-1 protein, which contains putative phosphorylation site of Plk-1. Immunofluorescence experiments indicated that S198 phosphorylation of Elk-1 appears to be localized to spindle poles during metaphase and translocate to the midbody during cytokinesis.

Conclusion: The interaction between Elk-1 and mitotic kinase Plk-1 indicates a potential role of Elk-1 during mitosis.

Keywords: Elk-1, mitosis, cancer, Plk-1

\section{P-021}

\section{ADAMTS and absance epilepsy}

Gürol G' ${ }^{1}$, Ekici $F^{2}$, Arabacı Tamer S ${ }^{1}$, Sar1 $S^{1}$, Demircan K

'Department of Physiology, Sakarya University, Faculty of Medicine, Sakarya, Turkey; ${ }^{2}$ Department of Physiology, YIldırım Beyazıt University, Faculty of Medicine, Ankara, Turkey; ${ }^{3}$ Department of Medical Biology, Turgut Ozal University, Faculty of Medicine, Ankara, Turkey 
Objective: Epilepsy is a chronic neurological diserder characterized by recurrent seizures that about $1 \%$ of the population worldwide. The cellular and molecular mechanisms of epilepsy have emerged to include immunological synapses, the extracellular matrix and the neurovascular unit. Different components of the extracellular matrix (ECM) contribute to many components of tissue reorganization during epileptogenesis and the ECM also play important role in regulation of synaptic excitability. ADAMTS (a disintegrin and metalloprotease with thrombospondin motifs) proteases comprise the most recently discovered branch of the extracellular metalloenzymes. ADAMTSs interact with extracellular matrix components. Although there are many studies in the literature, It is effects on epilepsy has not been investigated yet.

Methods: In this study, we used epileptic 6-months-old male WAG/Rij ( $\mathrm{n}=7)$ rats and nonepileptic 6-months-old male Wistar control $(n=7)$ rats. All rats were decapited after that cortex and thalamus were dissected. ADAMTS- 9 and 15 protein expression changes were performed using standard techniques of Western blot between experimental and control groups. The Student's t test was used for statistical evaluation of data (SPSS 15.00). p values $<0.05$ accepted as statistically significant.

Results: According to the preliminary findings of this study; 6 months WAG / Rij rats compare to 6-month Wistar control rats, there was no statistically significant difference in the cortex and thalamus tissue.

Conclusion: Further molecular and electrophysiological studies are needed in order to fully understand the roles of ADAMTS-9 and 15 expression in the pathogenesis of epilepsy.

Keywords: epilepsy, ADAMTS, WAG/Rij

\section{P-022}

Effect of valproic acid on the expression of P90RSK and $\mathrm{S} 6$ ribosomal proteins in Parkinson rat model

\section{Dagdelen $\mathrm{M}^{1}$, Eyuboglu $\mathrm{S}^{2}$, Yilmaz B ${ }^{2}$, Genc E ${ }^{1}$}

${ }^{1}$ Department of Medical Pharmacology, Yeditepe University Faculty of Medicine, Istanbul, Turkey; 'Department of Physiology, Yeditepe University Faculty of Medicine, Istanbul, Turkey

Objective: Parkinson's disease (PD) is characterized by the progressive degeneration of the nigrostriatal dopaminergic pathway. Many studies indicate the neuroprotective effects of valproic acid (VPA) in various neurodegenerative disorders. Ribosomal S6 kinase (RSK) is a family of serine threonine kinase which has important functions in cell proliferation, differentiation and survival. In the present study, we aim to determine the underlying mechanism of the effects of VPA on neuronal survival in a 6-hydroxydopamine (6-OHDA) animal model of Parkinson's disease.

Methods: Adult male Wistar albino rats were randomly divided into 4 experimental groups: Sham operated (S), sham operated and valproic acid treated (SV), 6-OHDA lesioned (PD) and 6-OHDA lesioned and valproic acid treated (PV). All rats were subjected to stereotaxic injection of 6-OHDA or saline to the right substantia nigra pars compacta. Rats were intraperitoneally injected with either valproic acid $(300 \mathrm{mg} / \mathrm{kg})$ or saline for 10 days. Phospho p90RSK (Ser380), phospho S6 ribosomal protein (Ser235/236) expression levels were determined from striatal tissue with western blotting method.

Results: VPA treatment increased phospho-p90RSK protein level in SV group compared to $\mathrm{S}$ and PD groups $(\mathrm{p}<0.05)$. Similarly phospho-p90RSK protein level was increased in PV group compared to PD group $(\mathrm{p}<0.05)$. Accordingly, VPA treatment increased phospho-S6 ribosomal protein level in SV group compared to $\mathrm{S}$ group. Similarly phospho-S6 ribosomal protein level was increased in $\mathrm{PV}$ group compared to $\mathrm{PD}$ group.

Conclusion: These results indicate that VPA may have neuroprotective effects through activation of survival pathways as p90RSK against 6-OHDA induced neuronal cell death.

Keywords: Parkinson's disease, valproic acid, p90RSK, S6 ribosomal protein

\section{P-023}

\section{Cloning and molecular characterization of a putative sodium channel in an invertebrate species}

\section{Coșkun C, Puralı N}

Department of Biophysics, Hacettepe University Faculty of Medicine, Ankara, Turkey

Objective: Most electrophysiological studies have been performed in invertebrate species and rather frequently in crayfish preparations due to less complex structure and relative ease in experimentation. Though, the kinetic properties of the voltage gated sodium channel (VGSC), in crayfish (Astacus leptodactylus) neurons, have been investigated however, the gene coding the channel protein is yet unknown. This study aimed at cloning the VGSC gene and to define the molecular properties of the related protein.

Methods: RNA were isolated from ganglia, cDNA copies were synthesized by RT-PCR. Gene fragments were amplified by a PCR using synthesized cDNA and degenerate primers, designed by considering the similarity within the genes in neighboring species. RACE (rapid amplification of cDNA ends) method were used for amplification of ends of the gene. Nucleotide sequence has been converted to a.a. sequences for molecular characterization of the protein.

Results: A 5206 bp of the gene including start codon was cloned. It corresponds to $89 \%$ of predicted gene size. Converted a.a. sequence is $81 \%$ similar to that of Cancer borealis. Transmembrane topology analysis demonstrated that the protein is a typical VGSC, having 4 distinct domains each one of which has 6 transmembrane regions.

Conclusion: In the present work, gene coding VGSC in Astacus leptodactylus is firstly explored. The cloned part of gene shows similarity to other genes in neighboring species. The study which is the first attempt to clone an ion channel, 
would enhance our interest in future channelopathy studies. (Grants: TUBITAK 113s555, HU BAB 13D031011003, 14D08101006).

Keywords: crayfish, ion channel, cloning

\section{P-024}

\section{Microarray analysis of Pea3 transcription factor regulation in SH-SY5Y cells}

$\underline{\text { Kandemir B }}^{1}$, Dağ $\mathrm{U}^{2}$, Durasi $\mathrm{MI}^{3}$, Güngör Bakır B ${ }^{4}$, Sezerman $\mathrm{U}^{3}$, Yilmaz B ${ }^{5}$, Aksan Kurnaz $\mathrm{I}^{6}$

${ }^{1}$ Department of Genetics and Bioengineering, Yeditepe University, Istanbul, Turkey; ${ }^{2}$ Howard Hughes Medical Institute, Janelia Farm Research Campus, Ashburn, VA, USA; ${ }^{3}$ Department of Molecular Biology, Genetics, and Bioengineering, Sabanci University Faculty of Biological Sciences and Bioengineering, Istanbul, Turkey; ${ }^{4}$ Department of Computer Engineering, Abdullah Gül Universtiy, Faculty of Engineering and Natural Sciences, Kayseri, Turkey; ${ }^{5}$ Department of Physiology, Yeditepe University Faculty of Medicine, Istanbul, Turkey; ${ }^{6}$ Department of Molecular Biology and Genetics, Gebze Technical University, Kocaeli, Turkey

Objective: The purpose of this study is to determine effect of Pea3 on gene expression by microarray analysis.

Methods: SH-SY5Y cell line was transfected with either Pea3VP16 (constituvely active) expression vector or Pea3ENG (expression supressor). Empty vector (pcDNA3) was used as control. Microarray analysis was performed to isolated RNA from cells. Various bioinformatics tools were used to evaluate the data of microarray.

Results: In this study, Pea3 overexpressed cell was compared with Pea3 suppressed cell based on the number of genes. It was shown that Pea3 overexpression was effect on more expression of genes and the number of gene was reduced while Pea3 was repressed.

Conclusion: Pea3 is ETS family transcription factor that regulates many genes. In previously study, we found that Pea3 promotes axonal outgrowth in PC12 and NSC34 cell line. In this study, it was determined that $\mathrm{Pea} 3$ regulates thousands of genes by microarray analysis in SH-SY5Y. In gene expression regulation, it has repressor property was shown.

Keywords: Pea3, transcription factor, microarray analysis

\section{P-025}

Effect of $\gamma$-glutamyl cysteinyl ethyl ester on mitochondrial DNA damage in excitotoxicity model

\section{Çizmeci G ${ }^{1}$, Yalçın A², Azak B ${ }^{1}$, Turunç Bayrakdar ES ${ }^{2}$}

'Department of Neuroscience, Ege Universityl Health Science Institute, Izmir, Turkey; ${ }^{2}$ Department of Biochemistry, Ege University Faculty of Pharmacy, Izmir, Turkey

Objective: Glutamate induced excitotoxicity is a triggering factor that causes neurodegeneration underlying nervous system disorders like epilepsy and stroke. Overstimulation of glu- tamate receptors results in production of reactive oxygen species (ROS). Kainic acid (KA), analogue of glutamate, can induce excitotoxicity in brain regions. Glutathione $(\mathrm{GSH})$ is crucial for the cellular defence against ROS. It is suggested that deficiency of GSH and excitotoxicity lead to mitochondrial damage in brain. For this reason we aimed to investigate protective effects of $\gamma$-glutamyl cysteinyl ethyl ester (GCEE), GSH precursor, on mtDNA damage in KA-induced excitotoxicity.

Methods: In our study, Sprague-Dawley rats were used; divided into three groups as control $(\mathrm{n}=4), \mathrm{KA}(10 \mathrm{mg} / \mathrm{kg})(\mathrm{n}=4)$ and $\mathrm{KA}+\mathrm{GCEE}(10 \mathrm{mg} / \mathrm{kg}+150 \mathrm{mg} / \mathrm{kg})(\mathrm{n}=4)$. Cerebellum and cortex samples were used for determination of mtDNA damage by Long PCR and diphenylamine (DPA) DNA fragmentation methods.

Results: KA treatment significantly increased the mtDNA damage in cerebellum assessed by DPA and long PCR methods when compared to control group. In addition GCEE treatment against KA significantly decreased the mtDNA damage in cerebellum when compared to KA group. No significant differences were found between groups in cortical samples.

Conclusion: It is concluded that KA leads to mtDNA damage in cerebellum and GCEE treatment may be helpful as a neuroprotective agent because of its decreasing effect on mtDNA damage against neurodegenerative processes triggered by KA. Our study provides an innovation by searching the effect of KA on cerebellum which is a different brain region from the previous studies about KA-induced excitotoxicity.

Keywords: exitotoxicity, mtDNA damage, Long-PCR, GCEE, cerebellum

\section{P-027}

\section{Gene regulation by Elk-1 in neuronal cells under} normoxic and hypoxic conditions

\section{Sahin $\mathrm{E}^{1}$, Kandemir B ${ }^{1}$, Yilmaz B ${ }^{2}$, Aksan Kurnaz I ${ }^{3}$}

${ }^{1}$ Faculty of Engineering, Department of Genetics and Bioengineering, Yeditepe University, Istanbul, Turkey; 2Faculty of Medicine, Department of Physiology, Yeditepe University, Istanbul, Turkey; ${ }^{3}$ Department of Molecular Biology and Genetics, Gebze Technical University, Kocaeli, Turkey

Objective: Elk-1 belongs to TCF subfamily of ETS-domain transcription factors, and plays as a key component in several cellular processes. Our major goal in this study is to profile target gene expression changes in SH-SY5Y neuroblastoma cell line as a result of transcriptional activity of Elk-1 under both normoxic and hypoxic conditions through microarray analyses.

Methods: SH-SY5Y cells were transfected with constitutively activated Elk1-VP16 construct or empty pcDNA3 plasmid (control). Total RNAs were extracted from these two groups of cells at 0 hour and microarray procedure was carried. For the hypoxia microarray, cells were transfected with Elk1-VP16 only and incubated under normoxic (control) or hypoxic (1\% 
O2) conditions for 6 hours. Total RNA extractions were performed and used for microarray analysis. Data obtained from two microarray experiments were evaluated using various bioinformatics tools and qRT-PCR was performed for verification.

Results: The microarray analyses for both normoxia and hypoxia revealed that Elk-1 plays important roles in several cellular processes such as cell to-cell signaling, cellular movement, and cell death and survival by means of transcriptional activity. By performing promoter analyses, the presence of Elk-1 binding motif was examined and it was determined that numerous target genes in relevant pathways can be regulated directly.

Conclusion: The regulation of some pathways by Elk-1 transcription factor activity under either normoxia or hypoxia was determined via performed bioinformatic analyses. Specifically, it is of our interest that some key components in hypoxia signaling and pluripotency pathways were suggested as potential targets of Elk-1 activity.

Keywords: Elk-1, transcription factor, hypoxia, microarray

\section{P-028}

Neuroprotective effects of 20(S)-ginsenoside Rg3 in diabetic neuropathy

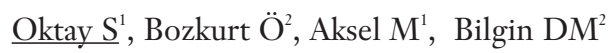

${ }^{1}$ Department of Biophysics, Adnan Menderes University Institute of Health Sciences, Aydin, Turkey; ${ }^{2}$ Department of Biophysics, Adnan Menderes University Faculty of Medicine, Aydın, Turkey

Objective: Ginseng is an herbal agent that has an antidiabetic, anti-inflammatory, antioxidant, and antiapoptotic activities. 20(S)-ginsenoside Rg3 (20(S)-Rg3), an active ingredient of ginseng saponins, has been reported to enhance insulin secretion in pancreatic beta cells and to have protective effects on diabetic renal damage. This study aims to evaluate the neuroprotective effects of $20(\mathrm{~S})-\mathrm{Rg} 3$ in experimental diabetic neuropathy.

Methods: Adult male Wistar rats $(\mathrm{n}=18)$ were randomly assigned for control, untreated diabetic and 20(S)-Rg3 treated diabetic groups. Diabetes was induced by a single intraperitoneal injection of STZ $(50 \mathrm{mg} / \mathrm{kg}), 3$ days after the STZ injection diabetes was assigned by the presence of hyperglycemia (>300 mg/dl). Rats in 20(S)-Rg3 treated group received $5 \mathrm{mg} / \mathrm{kg} /$ day $20(\mathrm{~S})-\operatorname{Rg} 3$ by oral gavage for 5 weeks. Body weights were monitored daily. Tail flick and hot plate tests were conducted to record nociceptive changes at 3rd and 4th week of treatment. Nerve conduction velocities (NCV) and distal latencies were determined in both sciatic nerves.

Results: STZ-induced diabetes caused a decrease in body weight while 20(S)-Rg3 treatment restored this decrement. Moreover, ginsenoside treatment significantly reduced plasma glucose levels compared to pretreatment states $(\mathrm{p}<0.05)$. Also 20(S)-Rg3 decreased both tail flick and hot plate latencies significantly in diabetic rats revealing its protection against noci- ceptive behavior. Diabetic rats showed a significant decrease $(\mathrm{p}<0.001)$ in NCV and distal latencies as compared to the control rats. However, NCVs and distal latencies were improved by the treatment of 20 (S) $-\operatorname{Rg} 3$.

Conclusion: In conclusion, these findings highlighted the possible neuroprotective effects of 20 (S)-Rg3 treatment on diabetic neuropathy.

Keywords: 20(S)-ginsenoside $\operatorname{Rg} 3$, diabetic neuropathy, nerve conduction velocity, nociceptive tests

\section{P-029}

The possible neuroprotective effects of pterocarposide treatment in experimental diabetic neuropathy

Bilgin DM ${ }^{1}$, Öztürk D², Sayarcan $\ddot{O}^{2}$, Bozkurt $\ddot{O}^{1}$

'Department of Biophysics, Adnan Menderes University Faculty of Medicine, Aydin, Turkey; 'Department of Biophysics, Adnan Menderes University Institute of Health Sciences, Aydın, Turkey

Objective: Pterocarboside is a naturally found C-glucoside derivative. Previous studies have demonstrated the positive effects of P. marsupium extract including pterocarboside on the treatment of noninsulin dependent diabetes. This study aims to evaluate the possible neuroprotective effects of pterocarboside treatment in experimental diabetic neuropathy.

Methods: Adult male Wistar rats $(\mathrm{n}=21)$ were randomly assigned for control, diabetic and pterocarboside treated diabetic groups. Pterocarboside used in the study was generously provided by Sabinsa Corporation. Diabetes was induced by a single intraperitoneal injection of STZ $(50 \mathrm{mg} / \mathrm{kg})$, and 3 days after the STZ injection the rats having hyperglycemia ( $>300$ $\mathrm{mg} / \mathrm{dl}$ ) were assigned to be diabetic. Rats in treatment group received a $25 \mathrm{mg} / \mathrm{kg} /$ day dose of pterocarboside by oral gavage for 21 consecutive days. Tail flick and hot plate tests were conducted to record nociceptive changes at 3 rd week of treatment. Nerve conduction velocities (NCV) and distal latencies were determined in sciatic nerves.

Results: The results revealed that pterocarboside treatment was not successful in restoring the diabetes-induced alterations in blood glucose and body weight. However, the elevated tail flick and hot plate latencies in diabetic group were observed to be reduced by pterocarboside treatment. Moreover, the diabetes-induced decrease in NCVs was improved by the treatment of pterocarboside. There was a significant $(\mathrm{p}<0.001)$ increase in the NCVs in comparison to the diabetic group.

Conclusion: In conclusion, these findings revealed the possible neuroprotective effects of pterocarboside treatment in diabetic neuropathy although the treatment was not successful in restoring the diabetes-induced blood glucose and body weight alterations.

Keywords: pterocarboside, diabetic neuropathy, nerve conduction velocity, nociceptive tests 


\section{P-030}

The effects of early postnatal cranial irradiation on the visual function of adult mice

$\underline{\text { Koç } T^{1}}$, Ergenoğlu T' $T^{2}$ Yllmaz BE ${ }^{3}$, Öztürk $\mathrm{CN}^{1}$, Öztürk $\mathrm{H}^{1}$

'Department of Anatomy, Mersin University Medical Faculty, Mersin, Turkey; ${ }^{2}$ Department of Physiology, Mersin University Medical Faculty, Mersin, Turkey; ${ }^{3}$ Department of Physiology, Mersin University Medical Faculty Research Hospital, Mersin, Turkey

Objective: Cranial radiotherapy is an effective tool in the treatment of pediatric malignancies but it is associated with adverse side effects, both short and long term. Especially, decreased visual acuity, radiation retinopathy, cataract and blindness are late onset effects in the eye and visual functions after cranial irradiation. In the current study, the possible effect of cranial irradiation on visual pathways is aimed to investigate by evaluating visual evoked potentials (VEP).

Methods: A group of 20 C57BL/6J female pups were used in this study. Single dose of 8? Gray (Gy) whole cranial irradiation at postnatal day 14 (P14) ( $\mathrm{Rad}+$ Group) or double doses ( $\mathrm{Rad}++$ Group) of 8 Gy both at P14 and P21 were administered to the pups. Additionally, a group of age and body weight matched mice were assigned as Sham group "anesthetic controls". Seven months after the cranial irradiation, by using pin electrodes visual evoked potential were recorded at the median occipital region of all groups of mice.

Results: The amplitude and latency values of VEP responds were measured and analyzed by One-Way ANOVA. The peakto-peak P2-N2 wave amplitude values of $\mathrm{Rad}++$ group were found to be significantly lower as compared to the Rad+ and Sham groups ( $\mathrm{p}=0.001$ and $\mathrm{p}=0.018$, respectively). In a similar trend, peak-to-peak N2-P3 wave amplitude values were significantly lower as compared to the Sham group $(\mathrm{p}=0.030)$.

Conclusion: Our overall data suggests that exposure of cranial irradiation to the young brain can cause to visual dysfunctions and this effect may be dose-dependent.

Keywords: visual evoked potential, cranial Irradiation, C57BL/6J, gray

\section{P-032}

Blood nerve barrier: investigatioin in rat sciatic nerve via different histological methods

Billur D, Bayram P, Kızıl Ş, Aydın S, Can B

Department of Histology and Embryology, Ankara University Faculty of Medicine, Ankara, Turkey

Objective: In this study, we aimed to investigate the bloodnerve barrier compartment (BNB), which provides the ionic environment continuity of the nerve fibers, with histological methods.

Methods: In our study, 6 male Albino Wistar rats' sciatic nerve were used. For light microscopy (LM) analyses, the slides were stained with Hematoxylen-Eosin (HE) and Mallory's Azan (MA) dyes. To show the presence of tight junctions (TJ) between perineural cells, we used anti-occludin and anti-ZO1 antibodies with immunofluorescent labeling. To investigate the ultrastructure of perineural cells we used routine tecniquies of transmission electron microscope (TEM).

Results: In LM analyses, we observed axonal structures, Schwann cells nuclei, endoneurial capillary, connective tissue fibers and perineurium which is composed of connective tissue surrounding the fascicles. In semi-thin sections we distinguished that perineural sheath formed by cellular layers. The expressions of occludin and ZO-1 between perineural cells showed us the excistence of TJ between these cells. In TEM analyses, perineural cell organization were formed concentric lamellar structure like around nerve fascicles where found unilayer in intrafascicular area. Perineural cells were connected to each other via junctional complexes. Amounts of pinocytic vesicles were observed in the cytoplasm. We observed non-fenestrated capillary in endoneurium. Collagen fibers and a small number of fibroblasts were observed around myelinated and unmyelinated axons.

Conclusion: According to our results, we demonstrated perineural cells of BNB by using ultrastructure immunofluorescent and methods.

Keywords: sciatic nerve, blood-nerve barrier, perineural cell

\section{P-033}

Protective role of melatonin on cobalt chloride induced chemical hypoxia model in SH-SY5Y cells

Uğuz CA ${ }^{1}$, Öz A1, Yılmaz B ${ }^{2}$, Altunbaş $S^{2}$, Çelik $\ddot{O}^{1}$

'Department of Biophysics, Süleyman Demirel University, Isparta, Turkey; ${ }^{2}$ School of Medicine Student, Süleyman Demirel University, Isparta, Turkey

Objective: Melatonin is a hormone, appointed to regulate circadian rhythm, which secreted by the pineal gland. Recent studies have showed that melatonin has also powerful antioxidant property and anticarcinogenic effects. SH-SY5Y neuroblastoma cell line is used for chemical hypoxia as well as neurological disease model of Parkinson's and Alzheimer's diseases. Cobalt chloride $(\mathrm{CoCl} 2)$ is an agent using experimental hypoxia model in various cell types. Hence in this study, we aimed to investigate the protective effects of melatonin on $\mathrm{CoCl} 2$ induced hypoxia model in SH-SY5Y neuronal cells.

Methods: First of all we performed cell viability (MTT) test to evaluate the effects $\mathrm{CoCl} 2$. Then, we performed intracellular ROS production, oxidative stress parameters, mitochondrial depolarization and caspase levels with spectrofluorometric methods by using plate reader.

Results: We determined that $\mathrm{CoCl} 2$ administration reduced cell viability by increasing the intracellular oxidation. We demonstrated increased ROS levels as a result of chemical hypoxia model of neuronal cells. Our results which obtained 
from mitochndrial depolarization levels also contributed to the results written above. Moreover, preincubation with melatonin prevents cells from apoptosis by decreasing Caspase -3 and -9 levels.

Conclusion: According to the results we obtained from our experiments, we can easily suggest that pre-incubation with melatonin protects neuronal cells from hypoxia induced apoptosis cascades.

Keywords: melatonin, CoCl2, hypoxia, SH-SY5Y neuroblastoma cells

\section{P-034}

\section{Relation between Parkinson's disease and DNA topoisomerase II, in neural-differentiated SH-SY5Y line}

$\underline{\text { Yeman B }}^{1}$, Karabulut $\mathrm{SD}^{2}$, Işık S

'Biology Master Program, Fatih University, Science and Engineering Institute, Istanbul, Turkey; 'Biotechnology PhD Program, Fatih University, Science and Engineering Institute, Istanbul, Turkey; ${ }^{3}$ Medical Biology Department, Fatih University, Faculty of Medicine, Istanbul, Turkey

Objective: DNA topoisomerase II, (topo II,) is a crucial enzyme in brain development and nerual differentiatiation. Inhibition of topo II, activity both in vivo and in vitro results in shorter axon length and increase in DNA damage. These findings indicate the important role of topo II, in axonogenesis. Axon shortening and degeneration seen in Parkinson's Disease (PD) may indicate a relation between PD and topo II, protein. So, the aim of this study is to determine possible relation between PD and topo II, expression levels in PD neuroblastoma cell line (SH-SY5Y).

Methods: SH-SY5Y neuroblastoma cell line induced with retinoic acid (RA) and BDNF (brain-derived neurotrophic factor) for neural differentiation, were used to establish PD model bu using MPP+. In this PD model, the presence of PD was confirmed via immunofluorescence staining of --synuclein protein. The mRNA samples, which were obtained from PD model, were used to acquire cDNA and these cDNA samples were amplified with gene specific primers to obtain relative gene expression levels of topo II,.

Results: The results were evaluated and these results were explained in bar graphs according to Pfaffl 2001. qPCR results demostrated that topo II, gene expression levels decreased in PD model SH-SY5Y cell line.

Conclusion: Neural differentiation is an extremely essential step to understand in order to contribute to the treatment of neurodegenerative diseases. According to our results, topo II, can be a neuroprotective gene and can be very important in the diagnosis and treatment of Parkinson's disease.

Keywords: DNA topoisomerase II,, aksonogenesis, Parkinson's disease, SH-SY5Y cell line

\section{P-035}

Medical illustration in neuroscience: a study with cadaver and illustration pictures

\section{Cümen $\mathrm{CE}^{1}$, Evren $\mathrm{M}^{2}$, Göde $\mathrm{S}^{3}$}

'School of Medicine, Ege University, Izmir, Turkey; '2Department of Biotechnology, Ege University Faculty of Natural and Applied Sciences, Izmir, Turkey; ${ }^{3}$ Deparment of Otolaryngology, Ege University School of Medicine, Izmir, Turkey

Objective: Medical illustration is an art-science division that enables the knowledge to be transferred correctly by visualizing scientific data. Sections such as neuroscience with dense theory and complex underlying mechanisms are difficult to understand and should be supported with illustrative figures. The aim of this study is to draw attention to the medical illustration's positive effect on comprehensibility through visualizing the cases of nerve damage in the process of cochlear implantation due to the variations of facial nerve settlement in an article to be published in an international publication.

Methods: Cadaver images taken in order to support the article "Revisiting The Anatomy of The Facial Recess: The Boundaries of The Round Window Exposure" were examined. In order to clarify the structure, drawings and diagrams were prepared. The use of computer hardware and software provides the flexibility that classical drawing techniques can not, and enables unlimited detail in images. Adobe Photoshop ${ }^{\circledR}$ software enabled us to produce illustrations using layers, reprocess and transfer digital images easily. US Logic Lapaaz ${ }^{\circledR}$ graphics tablet and a compatible PC were utilized to produce these illustrations.

Results: This study provides insights into how digital images produced using medical illustration techniques can enhance understanding of an article when utilized with cadaver images comparatively.

Conclusion: Medical illustration which is provided as graduate level education in some countries, has not received enough attention in our country. Medical illustration should also be represented at academic level in our country due to its unquestionable contribution to medical education and scientific research.

Keywords: medical illustration, cochlear implant, medical education

\section{P-036}

The tole of some oxidant-antioxidant parameters in pathogenesis of kidney damages in age dependent genetically absence epileptic rats

Aydemir B, Gürol G, Öğ̈ü S

Department of Biophysics, Faculty of Medicine, Sakarya University, Sakarya, Turkey

Objective: It is known that oxidative stress plays an important role in the pathophysiology of neurodegenerative diseases, 
such as Alzheimer's disease, Parkinson's disease and epilepsy. The aim of this study was to determine the levels of malondialdehyde (MDA), reduced glutathione (GSH) and nitric oxide (NO) in kidney tissues underlying genetically absence epileptic seizure in WAG/Rij rats.

Methods: 2 and 6-weeks old, 3 and 6-months-old female WAG/Rij ( $n=28)$ rats were used in our study. The levels of MDA, GSH and NO were determined by biochemical methods in all study samples. Results were compared with the oneway ANOVA and Tukey post-hoc tests.

Results: The kidney tissue levels of MDA in 6-mo old WAG/Rij rats were found to be significantly higher than the 2and 6-weeks old WAG/Rij rats. The kidney tissue levels of GSH in 6-weeks old WAG/Rij rats were found to be significantly higher than 3-and 6-mo old WAG/Rij rats. Additionally, no significant differences were found between the groups as NO levels in kidney tissues were taken into consideration.

Conclusion: From these results, it can be concluded that increased oxidative stress-induced damage of kidney tissues, probably due to reduced antioxidant system parameters, may be associated with increased seizure frequency and age dependent changes in WAG/Rij rats.

Keywords: epilepsy, kidney, oxidative stress

\section{P-037}

The oxidative stress status in liver tissue of age dependent genetically absence epileptic rats

Aydemir B, Gürol G, Öğüt S, Cinemre BF

Department of Biophysics, Faculty of Medicine, Sakarya University, Sakarya, Turkey

Objective: Epilepsy is one of the most common neurodegenerative disease, characterized by recurrent seizures, which may increase the content of free radicals. The aim of this study was to investigated the levels of malondialdehyde (MDA), reduced glutathione (GSH) and nitric oxide (NO) in liver tissues underlying genetically absence epileptic seizure in WAG/Rij rats.

Methods: 2 and 6-weeks old, 3 and 6-months-old female WAG/Rij $(n=28)$ rats were used in our study. The levels of MDA, GSH and NO were determined by biochemical methods in all study samples. Results were compared with the oneway ANOVA and Tukey post-hoc tests.

Results: The liver tissue levels of MDA in 3-and 6-mo old WAG/Rij rats were found to be significantly higher than the 2and 6-weeks old WAG/Rij rats. Moreover, no significant differences were found between the groups as NO and GSH levels in liver tissues were taken into consideration.

Conclusion: From these results, it can be thought to increased oxidative stress-induced damage of liver tissues, probably due to increased lipid peroxidation, may be associated with increased seizure frequency and age dependent changes in WAG/Rij rats.

Keywords: epilepsy, liver, oxidative stress

\section{P-038}

Effect of $\gamma$-glutamyl cysteinyl ethyl ester on specific miRNA expressions in excitotoxicity model

$\underline{\text { Azak B }}^{1}$, Yalçın A², Çizmeci G ${ }^{1}$, Turunç Bayrakdar ES

'Department of Neuroscience, Ege University Health Sciences Institute, Izmir, Turkey; ${ }^{2}$ Department of Biochemistry, Ege University Faculty of Pharmacy, Izmir, Turkey

Objective: The expression patterns and functions of miRNAs in neurodegenerative diseases have not been fully understood yet. Targeting miR-34 $\alpha$, miR-132 and miR-184 has been reported to alter seizure-induced neuronal death. Kainic acid (KA) is an analogue of glutamate and can induce excitotoxicity. In our study we aimed to investigate the effects of $\gamma$-glutamyl cysteinyl ethyl ester (GCEE), neuroprotective agent, on miRNA profiles in KA induced excitotoxicity.

Methods: In our study, Sprague-Dawley rats were used. Control group $(n=4)$ was given saline, KA group $(n=4)$ was given $10 \mathrm{mg} / \mathrm{kg} \mathrm{KA}$ and KA+GCEE group (n=4) was given 10 $\mathrm{mg} / \mathrm{kg} \mathrm{KA}$ and $10 \mathrm{mg} / \mathrm{kg}$ GCEE intraperitoneally. miRNA expressions were evaluated by Real-Time PCR in striatum and cerebellum.

Results: KA+GCEE and KA treatment significantly increased expressions of miR34., miR132 and miR184 in cerebellum when compared to control. In striatum, expressions of miR34. and miR132 in KA and KA+GCEE group were significantly increased when compared to control. Also the levels of expression were found higher in KA+GCEE group. However miR184 expression was decreased by both KA and KA+GCEE treatment in striatum.

Conclusion: It is observed that expressions of miR34., miR132 and miR184 influenced by excitotoxicity. Decreased miR184 expression with KA traetment may show that miR184 can be associated with neuronal survival. It is considered that increased miR34. and miR132 expressions can be caused by increase in oxidative stress that results in apoptosis and neurodegeneration. Our results showed that neuroprotective effect of GCEE was not related with effect on miRNA profiles in KA-induced excitotoxicity.

Keywords: excitotoxicity, GCEE, miRNA, striatum, cerebellum

\section{P-039}

The effects of the antioxidants in induced DM to the blood brain barrier and the inflammation

Bahcelioglu $M^{1}$, Take Kaplanoğlu $\mathrm{G}^{2}$, Gözil R${ }^{1}$, Buru ${ }^{\text {E1 }}$, Helvacioğlu $\mathrm{F}^{3}$, Çalgüner $\mathrm{E}^{1}$, Erdoğan $\mathrm{D}^{2}$

'Department of Anatomy, Gazi University, School of Medicine, Ankara, Turkey; 'Department of Histology and Embryology, Gazi University, School of Medicine, Ankara, Turkey; ${ }^{3}$ Department of Histology and Embryology, Başkent University, School of Medicine, Ankara, Turkey

Objective: Diabetes mellitus is a chronic metabolic disease with complications linked to oxidant stress, diminshed antioxi- 
dant defense of the body with decreased consumption of oxygene in mitochondrions. We aimed to investigate the possible effect of the antioxidant therapy such as green tea and vitamin E. The glial fibrillary acidic protein and nuclear factor $\kappa \beta$ activities were investigated to evaluate the inflamation and distribution of astrocytes.

Methods: 54 Wistar albino rats were divided into 9 groups. After 6 week of the streptozotocin injection, groups 7,8,9 received orally green tea and vitamin E for 4 weeks. Group 1: Normal control, Group 2: Na sitrat, Group 3: Green tea, Group 4: Vitamin E, Group 5: Green tea+vitamin E, Group 6: Diabetic control (single injection), Group 7: STZ+green tea, Group 8: STZ+Vitamin E, Group 9: STZ+green tea+vitamin E. At the end, all the animals were sacrified and immunohistochemical examination of anti-GFAP and anti-NF-ÎI, were done with Photo-light microscope.

Results: In diabetic control group, GFAP immunoreactivity was observed to be interrupted in pia-glial membrane and surrounding vessels. Administration of antioxidant agents were not sufficiently effective for the restructuring of the bloodbrain barrier. In diabetic control group, NFK $\beta$ immunoreactivity was very prominent in neurons and glial cells and antioxidant therapy decreased the immunoreactivity of $\mathrm{NF} \kappa \beta$ in these structures.

Conclusion: We observed an interrupted immunoreactivity of GFAP in pia-glial membrane and surrounding vessels of frontal cortex while $\mathrm{NF} \kappa \beta$ staining was increased in neurons and glial cells. We determined that the antioxidant theraphy was not effectively decreasing these effects observed in diabetic group.

Keywords: diabetes mellitus, blood brain barrier, antioxidant, GFAP, NFк $\beta$

\section{P-040}

Adult Ambystoma mexicanum (axolotl) spinal cord cell culture protocol and cell identification

Polat B, Kubat Öktem E, Akagündüz BÇ, Öztürk G

Regenerative and Restorative Medicine Research Center (REMER), Medipol University, Istanbul, Turkey

Objective: Spinal cord injury is quite a common condition in the society which leads to the death of the neurons and axons in the damaged region. The central nervous sytem has a limited capacity in human and damage made in spinal cord deteriorates in time. Studies demonstrate that during regeneration seen in salamanders, axon regrowth as well as cell differentiation occurs and these alterations continue throughout life. Ambystoma mexicanum (Axolotl), which has been studied with new approaches recently, is particularly studied in epimorphic and spinal cord regeneration and they are among the easiest species to reproduce. However, literature holds too few cell culture studies. In this study, we aim to optimize Axolotl spinal cord cell culture protocol and to begin to identify the cellular origins of high regenerative capacities of this model organism via basic immunocytochemistry.

Methods: For this purpose, the spinal cord tissue of a young adult axolotl was acquired and cells were dissociated enzymatically and mechanically. Cells were planted without any additional help of serum or factors. They were observed for seven days and then they were stained with various neuronal, glial and stem cell antibodies.

Results: As a result of this study, we have shown that the axolotl central nervous system cells have better survival capacity compared to the mammalian central nervous system cells. In addition to that, cell populations of high variety were observed.

Conclusion: In conclusion, our data shows that this protocol is a good model for correlative regeneration studies at cell culture level.

Keywords: axolotl, regeneration, cell culture, stem cell

\section{P-041}

The antioxidant effect of montelukast on experimental Huntington's disease model

Yüksel $\mathrm{M}^{1}$, Aydemir $\mathrm{S}^{2}$, Güçlü $\mathrm{H}^{1}$, Özkan $\mathrm{N}^{2}$, Erdoğan $\mathrm{N}^{2}$

${ }^{1}$ Department of Medical Laboratory Technicianship, Marmara University, Vocational School of Health Related Services, Istanbul, Turkey; ${ }^{2}$ Department of Pathology Laboratory Technicianship, Marmara University, Vocational School of Health Related Services, Istanbul, Turkey

Objective: 3-nitropropionic acid (3-NP), is a fungal toxin, inhibits succinate dehydrogenase in Krebs cycle and electron transport chain. Systemic administration of 3-NP to rats cause neuronal degeneration in striatum and cognitive deficits, like Huntington's disease. Montelukast (ML) is a leukotriene D4 receptor antagonist, which has a reducing effect on inflammation in various diseases. The aim of this study was to investigate the putative neuroprotective effect of ML against 3-NP induced rat model.

Methods: Female Sprague-Dawley rats were included in the study. Rats were divided into four groups; 3-NP group (20 $\mathrm{mg} / \mathrm{kg} /$ day), ML group (10 mg/kg/day), 3-NP-ML group and controls. After 10 days, rats were decapitated and brains were removed. We measure in brain tissues luminol and lucigenin enhanced chemiluminescence (CL) for free radical release, malondialdehyde (lipid peroxidation) and glutathione levels (an antioxidant) for oxidative stress determination and observed histopathological evaluations'.

Results: Superoxide radical generation was significantly higher in 3-NP group, with respect to control group, which was reduced with $\mathrm{ML}$ treatment $(15.6 \pm 1.4$ vs. $8.1 \pm 1.2$ vs. $9.9 \pm 1.1$ $\mathrm{rlu} / \mathrm{mg} ; \mathrm{p}<0.001)$. Luminol enhanced CL were increased in 3NP group with respect to controls $(15.3 \pm 2.1$ vs. $11.8 \pm 1,6$ $\mathrm{rlu} / \mathrm{mg} ; \mathrm{p}<0.05)$. ML treatment reduced the release of $\mathrm{OH}$, $\mathrm{H} 2 \mathrm{O} 2$ and $\mathrm{HOCl}-$ radicals $(11.0 \pm 2.2 \mathrm{rlu} / \mathrm{mg} ; \mathrm{p}<0.01)$. MDA levels were higher and GSH levels were lower in 3-NP group. 
ML treatment ameliorates the effects. Histopathologic observations show that striatal lesions after $3-\mathrm{NP}$ induction are reduced with $\mathrm{ML}$ treatment.

Conclusion: In conclusion, ML treatment reduces free radical induced oxidative stress lesions in 3-NP induced experimental Huntington's disease model.

Keywords: 3-nitropropionic acid, montelukast, oxidative stress, Huntington's disease

\section{P-042}

Investigation of roles of FGF, B27 and FCS in survival cerebral cortex of newborn mouse

$\underline{\text { Ayșit } N^{1}}$, Bahçeci $\mathrm{B}^{2}$, Öztürk $\mathrm{G}^{1}$

'Regenerative and Restorative Medicine Research Center (REMER), Istanbul Medipol University, Istanbul, Turkey; ${ }^{2}$ Medical School, Istanbul Medipol University, Istanbul, Turkey

Objective: The aim of this study rolls of B27, FGF and FGF neuron survival in postnatal serebral cortex of mice.

Methods: Postnatal serebral cortex of mice was dissectioned with cold L15 medium. After enzymatic and mechanic dissection neuron was set in polyL-lizin petri dish with NBA which has no serum and factors, Then FGF. FCS, B27 and propidium iyodüre was added in this petri dish and inkübeded.The cells fixed after 72 hours were additionally stained with propidium iodide to determine dead / live cells with confacal microscope.

Results: Made in this study obtained by FCS added petri dishes and compared to the other groups the number of surviving cells was found to be higher.

Conclusion: Newborn mice a change was seen in the cerebral cortex contrary to popular belief B27 added with neurons in petri dishes regeneration. FCS and FGF were added to the plates for as well as the litarür a correlated increase neuronal survival.

Keywords: oostnatal neuron, cerebral cortex, B27, FGF, FCS

\section{P-043}

\section{A case of myasthenia with extremity involvement}

Kotan D ${ }^{1}$, Gürol G' ${ }^{2}$, Alemdar $M^{3}$, Arabac1 Tamer S

'Department of Neurology, Sakarya University, Faculty of Medicine, Sakarya, Turkey; 'Department of Physiology, Sakarya University, Faculty of Medicine, Sakarya; ${ }^{3}$ Department of Neurology, Sakarya University Education and Research Hospital, Sakarya, Turkey

Objective: Myasthenia gravis (MG) is autoimmune disease which developing antibody-mediated against the nicotinicacetylcholine-receptor(AChR) in postsynaptic membrane. MG starts with $50 \%$ ocular signs and is seen $90 \%$ ocular symptoms in course of disease. Goes to $10 \%$ of patients with extremity involvement as an initial manifestation but limited to the extremities is rare. Here, the case whose findings limited to extremities even four years after the onset of symptoms is presented.
Methods: 28-year-old female patient was admitted to our clinic with extreme weakness, fatigue, lifting arms up, climbing stairs and complaints of difficulty getting up from seat in the evening and on some days of the last six months. The patient was diagnosed with neurological inspection, electrophy siological, serologic test.

Results: Feature wasn't detected in the self and family history. Neurological examination; neck flexion (5-/5), the upper extremity proximal muscle strength (4/5), lower extremity proximal muscle strength $(4+/ 5)$. Thyroid function tests, level of B12, folate levels, serological tests were normal limits. Nerve conduction velocities were normal, also observed dekremental response in ulnar nerve. Pathology wasn't detected in cranial MRI and thorax BT. In case, marked improvement in muscle strength was observed at $60 \mathrm{~min}$ in tests with pyridostigmine bromide tablets $(120 \mathrm{mg})$. Symptoms of case which received corticosteroid therapy provided lasting improvement.

Conclusion: A clinical situation suggests strongly MG in cases which leads to confusion betimes. Our case with limb onset and increase in symptoms was reached diagnosis by electrophysiological and serologic findings. In this presentation, rare MG's limb was meant to draw attention to involvement and emphasized to importance of help diagnosis in clinical which would be misleading in these situations.

Keywords: myasthenia gravis, acetylcholine receptor, neuromuscular disease

\section{P-044}

\section{A case of multiple sclerosis interfering with toxic optic neuropathy}

Kotan $\mathrm{D}^{1}$, Alemdar $\mathrm{M}^{2}$, Gürol G ${ }^{3}$, Olcaysu $\mathrm{OO}^{4}$, $\underline{\text { Arabac1 Tamer } \mathrm{S}^{3}}$

'Department of Neurology, Sakarya University, Faculty of Medicine, Sakarya, Turkey; 'Department of Neurology, Sakarya University Education and Research Hospital, Sakarya, Turkey; ${ }^{3}$ Department of Physiology, Sakarya University, Faculty of Medicine, Sakarya, Turkey; ${ }^{4}$ Eye Diseases, Area Education and Research Hospital, Erzurum, Turkey

Objective: Multiple sclerosis (MS) is partly associated with genetic and environmental factors, is a disease of autoimmune origin. MS is often affected optic nerve and intraocular inflammation, uveitis, retinal vasculitis may show in MS. Here, patients who is diagnosed as MS with vision problems in 6 month of ethambutol therapy is presented.

Methods: 41-year-old-male-patient was evaluated in consultation with right eye pain began a month ago and loss of vision in both eyes. The resume, the diagnosis of tuberculosis 7 months ago, ethambutol, pyrazinamide, rifampicin and isoniazid treatment received for 6 months, ethambutol and pyrazinamide was interrupted 20 days ago, treatment has been continued with dual anti-tuberculosis treatment.

Results: There was no feature except viability on deep tendon reflexes in neurological examination. In fundus examination, 
left optic disc was natural appearance, but edema was determined with optical coherence tomography (OCT) and optic nerve head analysis in nasal, inferior and superior quadrant. We detected edema in all quadrants and optic disc margins were irregular in right optic disc nasal. There was widespread defect and scotoma in visual field. P100 latency prolonged in vizuel evoked potential examination. Hyperintense lesions were determinated compatible with alarge number of demyelinating plaques in cranial magnetic resonance imaging. Vasculitis markers and CSF examination was normal, oligoclonal band was negative. Although there was no improvement in symptoms of patient to take medication during follow-up. MS suspected cases, interferon treatment was started.

Conclusion: It is necessary where optic nerve is affected by central nervous system and were asked to draw attention to importance of imaging in differential diagnosis.

Keywords: multiple sclerosis, toxic optic neuropathy, neurological diseases

\section{P-045}

\section{Effects of fasudil on amyloid-beta induced cytotoxicity and apoptosis}

Uslu Kuzudişli S , Öztaş N² , Erdoğan $\mathrm{S}^{3}$, Doğantekin E${ }^{4}$, Demiryürek $\mathrm{T}^{5}$, Koç A ${ }^{6}$, Eser $\mathrm{EB}^{2}$

'Department of Neurology, Emine-Bahaeddin Nakıboğlu Medical Faculty, Zirve University, Gaziantep, Turkey; '2Department of Biochemistry, Emine-Bahaeddin Nakıboğlu Medical Faculty, Zirve University, Gaziantep, Turkey; ${ }^{3}$ Department of Medical Biology, Medical Faculty, Trakya University, Edirne, Turkey; ${ }^{4}$ Department of Microbiology, Emine-Bahaeddin Nakıboğlu Medical Faculty, Zirve University, Gaziantep, Turkey; ${ }^{5}$ Department of Pharmacology, Medical Faculty, Gaziantep University, Gaziantep, Turkey; ${ }^{6}$ Department of Histology and Embryology, Medical Faculty, Mevlana University, Konya, Turkey

Objective: Alzheimer's disease comprises the most frequent reason of demans. Aggregation of amyloid-beta (A $\beta)$ plaques and formation of neurofibrillary tangles are the basic changes in the pathogenesis of this disease. It is claimed that inhibiton of Rho-kinases may be a potential therapeutic agent in inflammatory and demyelinating diseases. The purpose of this study is to investigate the possible protective/therapeutic effects of fasudil, a Rho kinase inhibitor, on cytotoxicity and apoptosis by A $\beta$-induced.

Methods: Apoptotic neuronal death was induced in PC12 cells by the administration of $A \beta 25-35$. In evaluating levels of neuronal injury and the potential protective effect of fasudil, MTT cell viability assay was performed. Real-time PCR method has been used for the evaluation of apoptotic cell death. Nitric oxide levels of cell culture medium were measured using NO/ozone chemiluminescence technique.

Results: Fasudil administration was shown to protective effect on cell viability in A $325-35$ induced neuronal cell death. Realtime PCR method revealed that fasudil had an anti-apoptotic effect in this experimental model. A reduction bax and caspase3 mRNA levels was observed after fasudil administration. NO significant difference was observed in nitric oxide levels between experimental groups.

Conclusion: Fasudil was demonstrated to have a cell protective effect in neurotoxicity induced by A $\beta 25-35$ and to diminish the apoptosis by decreasing activation of bax and caspase- 3 that promotes apoptosis. These results suggest that fasudil may have a role in management of amyloid beta neurotoxicity in Alzheimer's disease. This study was supported by TÜBİTAK (Grant no: 113S952).

Keywords: fasudil, amyloid-beta, PC12 cells, apoptosis, neurotoxicity

\section{P-046}

Effects of Cichorium intybus on seizure development in pentyleneterazole kindling model of epilepsy

Ergül Erkeç $\ddot{O}^{1}$, Meral $\dot{I}^{2}$, Kara $M^{1}$, Eşrefoğlu $M^{3}$, Tok $\mathrm{EO}^{3}$

'Department of Physiology, Yuzuncu Yil University, Faculty of Medicine, Van, Turkey; 'Department of Physiology, Bezmialem Vakif University, Faculty of Medicine, Istanbul, Turkey; ${ }^{3}$ Department of Histology and Embriology, Bezmialem Vakif University, Faculty of Medicine, Istanbul

Objective: Epilepsy is one of the most common disorder that affects approximately $1 \%$ of the general population. Cichorium intybus roots have been traditionally used to cure epilepsy in Eastern Anatolia. The aim of this study is to investigate the effects of Cichorium intybus aqueous extract on epileptogenesis.

Methods: 27 Wistar albino male rats were randomly divided into 3 groups as control, epilepsy, and plant extract treated groups. After each PTZ injection the convulsive behaviour was observed for 30 min during kindling procedure. After completion of the behavioural experiments, GABAA receptor density and GABAA receptor positive cell number were evaluated immunohistochemically in whole brain and hippocampus.

Results: In Cichorium intybus aqueous extract administered group, occurrence of epileptic seizure activity was found more delayed compared to PTZ group. There was a significant difference in whole brain for GABAA receptor staining density and for GABAA receptor protein cell number in hippocampus between groups.

Conclusion: Cichorium intybus plant treatment has positive effects on retarding epileptogenesis and keep GABAA receptor positive hippocampal cell number close to control group despite PTZ application.

Keywords: Cichorium intybus, epilepsy, GABAA, pentylenetetrazol kindling, immunohistochemistry 


\section{P-047}

\section{Molsidomine reduces penicillin induced epileptiform activity}

Kocacan ES ${ }^{1}$, Marangoz $\mathrm{HA}^{2}$, Him A $^{3}$, Marangoz $\mathrm{C}^{1}$

'Department of Physiology, Ondokuz Mayis University Faculty of Medicine, Samsun, Turkey; 'Department of Neurosurgery, Ondokuz Mayis University Faculty of Medicine, Samsun, Turkey; ${ }^{3}$ Department of Biophysics, Ondokuz Mayis University Faculty of Medicine, Samsun, Turkey

Objective: Molsidomine is a nitric oxide (NO) donor. NO is a small gas molecule found in many tissues and can diffuse through membranes. NO is though to play a role in pathophysiology of epilepsy, though it is conflicting. The aim of the study is to investigate the effects of molsidomine on the epileptiform activity induced by penicillin.

Methods: In this study adult male Wistar rats weighing $220 \pm 30 \mathrm{~g}$ were used $(n=40)$. The left cerebral cortex was exposed by craniotomy under urethane anesthesia $(1.25 \mathrm{~g} / \mathrm{kg})$. The epileptiform activity was induced by microinjection of 500 IU penicillin $\mathrm{G}$ sodium salt $(200 \mathrm{IU} / 1 \mu \mathrm{l})$ into the left lateral ventricle. Molsidomine was given at doses of 50, 100 or 200 $\mathrm{mg} / \mathrm{kg}$ intraperitoneally 30 minutes before penicillin $\mathrm{G}$ sodium salt injection.

Results: Molsidomine $(50 \mathrm{mg} / \mathrm{kg}$ ) significantly reduced the spike frequency in the first 40 minutes and between 125th and 130th minutes after penicillin injection $(\mathrm{p}<0.05)$. Molsidomine $(100$ $\mathrm{mg} / \mathrm{kg}$ ) significantly reduced the spike frequency in the first 10 minutes $(\mathrm{p}<0.05)$. Molsidomine $(200 \mathrm{mg} / \mathrm{kg})$ significantly decreased the spike frequency in the first 5 minutes $(\mathrm{p}<0.05)$. Also 50 and $100 \mathrm{mg} / \mathrm{kg}$ doses of molsidomine significantly extended the latency of epileptic activity $(\mathrm{p}<0.05)$. Molsidomine groups showed no significant differences in terms of latency and spike frequency when compared with each other $(\mathrm{p}>0.05)$.

Conclusion: The present study showed that molsidomine suppressed the epileptiform activity induced by penicillin. This result suggests that NO may be an endogenous anticonvulsant substance.

Keywords: molsidomine, nitric oxide, epilepsy, penicillin, rat

\section{P-048}

The antienflamatuar effects of apelin to astrocytes that stimulated with lipopolisaccaride

Doğantekin $E^{1}$, Yener NB ${ }^{2}$, Erdoğan $\mathrm{S}^{3}$, Uslu Kuzudişli $\mathrm{S}^{4}$

'Department of Microbiology, Emine-Bahaeddin Nakıboğlu Medical Faculty, Zirve University, Gaziantep, Turkey; '2Department of Biochemistry, Emine-Bahaeddin Nakıboğlu Medical Faculty, Zirve University, Gaziantep, Turkey; ${ }^{3}$ Department of Medical Biology, Medical Faculty, Trakya University, Edirne, Turkey; ${ }^{4}$ Department of Neurology, Emine-Bahaeddin Nakıboğlu Medical Faculty, Zirve University, Gaziantep, Turkey

Objective: Cell damage by neuroinflammation in the CNS because of the activation of microglia and astrocytes is important for the pathogenesis and the prognosis of norodegenera- tive diseases. The aim of this study is research off the apelin's protective and antienflamatuar effects on the astrocytes which stimulated with LPS.

Methods: In this research we modelled neuroinflamation in C8D1-A mouse astrosit cell lines. We used LPS to develop neuroinflamation. We used MTT test to evaluate cell dejeneration and protective effects of apelin. We used real time qRTPCR to detect antiinflammatory effects of apelin.

Results: We treated C8D1-A cell lines with LPS and apelin in different time courses and doses. We did not detect any cytotoxic effects on these cell lines. On the other hand we detected that the levels of proinlammatory cytokins like IL-1 beta, TNF-alpha and IL-6 were increased when astrocytes were stimulated by LPS. The levels of these cytokines were supressed when cells were treated with apelin. Apelin also decreased the level of IL-10, which is an antiinflammatory mediator.

Conclusion: Apelin does not have any cytotoxic effects on astrocytes. Apelin decreases the synthesis of proinflammatory cytokines like IL-1 beta, TNF-alpha and IL-6. Behind this apelin increases the levels of IL-10 and has antiinflammatory effects. These results suggest that, more investigations and researches can be planned about apelin. Apelin may take a part in the treatment neuroinflammation which is very important in pathogenesis of neurodejenerative diseases. This study is supported by TUBITAK.

Keywords: apelin, LPS, C8D1-A cell line, inflammation

\section{P-049}

\section{Proconvulsant effect of Ginkgo biloba on the seizures induced by pentylenetetrazole in rats}

Yildırım $\mathrm{H}^{1}$, Yildırım $\mathrm{M}^{2}$, Ayar $\mathrm{A}^{2}$

'Department of Physiology, Karadeniz Technical University Institute of Health Sciences, Trabzon, Turkey; 'Department of Physiology, Karadeniz Technical University Faculty of Medicine, Trabzon, Turkey

Objective: Ginkgo biloba is one of the widely used medicinal plants for cognitive impairment and dementia. The present study aimed to investigate the effect of Ginkgo biloba extract (EGb 761) on pentylenetetrazole (PTZ)-induced seizures in rats.

Methods: Male Wistar rats (8 weeks old) were divided into five groups and were treated with EGb 761 (50, 100 and 200 $\mathrm{mg} / \mathrm{kg}$ ), carbamazepine ( $40 \mathrm{mg} / \mathrm{kg}$ ) or tap water by oral gavage for 7 days. Epileptic seizures were induced by an intraperitoneal injection of $60 \mathrm{mg} / \mathrm{kg}$ PTZ. Seizure intensity was scored using a modified Racine's scale during the first 30 min after PTZ injection.

Results: Pretreatment with $200 \mathrm{mg} / \mathrm{kg}$ EGb 761 significantly increased the mortality caused by PTZ (p=0.026). EGb 761 also caused a dose-dependent increase in the duration and severity of seizures induced by PTZ, but this effect was not significant. On the other hand, carbamazepine completely prevented the deaths caused by epileptic seizures. 
Conclusion: These results indicate that Ginkgo biloba extract EGb 761 can increase the susceptibility to PTZ-induced seizures in rats. For a more conclusive statement, it could be suggested that the other experimental epilepsy models could be used to examine the potential adverse effects of EGb 761 on the epileptic seizures.

Keywords: Ginkgo biloba, pentylenetetrazole, seizure, rat

\section{P-050}

The effects of melatonin application on nervous system at experimental epilepsy model made by PTZ

Sirinyıldız F', Ek RO'1, Cesur G', Yıldız $Y^{1}$, Ergin $K^{2}$

${ }^{1}$ Physiology Depatment, Adnan Menderes University Faculty of Medicine, Aydın, Turkey; ${ }^{2}$ Histology Embryology Depatment, Adnan Menderes University Faculty of Medicine, Aydın, Turkey

Objective: The purpose of this study is to examine of central nervous system changing parameters, metabolic changing parameters and peripheric central sysytem changing parameters between control group, epileptic group which is induced by Pentylenetetrazole (PTZ) and melatonin treatment groups $(25 \mathrm{mg} / \mathrm{kg}$ and $100 \mathrm{mg} / \mathrm{kg})$. The examinations were done both intragroups and intergroups.

Methods: 40 wistar albino rat that are male and 12-14 week old divided into 4 groups (Kontrol, PTZ, MEL25, MEL100). Only solvent was injected to rats from Kontrol group and $35 \mathrm{mg} / \mathrm{kg}$ dose of PTZ was injected to rats from PTZ group every other day total 23 days and 12 injections. $25 \mathrm{mg} / \mathrm{kg}$ melatonin +35 $\mathrm{mg} / \mathrm{kg}$ dose PTZ was injected to rats from MEL25 group and $100 \mathrm{mg} / \mathrm{kg}$ melatonin $+35 \mathrm{mg} / \mathrm{kg}$ dose PTZ was injected to rats from MEL100 group. Melatonin was injected half hour before PTZ injection and after PTZ injection rats were monitored for one hour. All doses injected intraperitoneally. The parameters that we looked for were; histologically cerebellum evaluation (4X and 10X microscobic photograph, last day after decapitation), hot plate nociceptive test (second, 19th day), epileptic seizure power scale (scores, every PTZ injection days).

Results: We found statically significant data for seizure scores. However there is no significant data for the tests histological evalution of cerebellum, hot plate nociceptive test (Significans value $=\mathrm{p}<0.05)$.

Conclusion: Our study showed that, melatonin has protective and healing power againist physiological changes that caused by epileptical seizures.

Keywords: pentylenetetrazole, rats, experimental epilepsy, melatonin, seizure scoring

\section{P-051}

\section{The role of glucocorticoids in rat model of} post-traumatic stress disorder

\section{Tanriverdi $\mathrm{AM}^{1}$, Cabadak $\mathrm{H}^{1}$, Gören $\mathrm{MZ}^{2}$}

'Department of Biophysics, Marmara University Faculty of Medicine, Istanbul, Turkey; 'Department of Pharmacology, Marmara University Faculty of Medicine, Istanbul, Turkey
Objective: Post-traumatic stress disorder is aa anxiety disorder where the subjects re experience the trauma with a reminder Steroids have important roles during stress has been shown in previous studies. In this study, the role of steroids on anxiety index were studied using a steroid prednisolone and steroid synthesis inhibitor metyrapone.

Methods: Female Sprague-Dawley weighing 200-250 g were adopted to reverce light-dark cycle received physiological saline (PS; $0.1 \mathrm{ml} / 100 \mathrm{~g})$, prednisolone $(20 \mathrm{mg} / \mathrm{kg})$ or metyrapone $(100 \mathrm{mg} / \mathrm{k})$. Following injections, the rats were subjected to dirty cat litter in plexiglass cage for 10 minutes. One week following trauma, the rats were subjected to clean cat litter that served as trauma reminder, the behavioral parameters were calculated in elevated plus maze test. All procedures were made under dim red light and the anxiety index was calculated for each rat according the video recordings.

Results: In the non-traumatized control group the anxiety index was calculated as 0.63 , traumatized controls anxiety index was calculated as 0.86 . Two-way analysis of variance indicated a variance and an interaction was found between trauma and treatments $(\mathrm{p}<0.0001)$. Bonferoni post-test showed the PS treatment caused the variance $(\mathrm{p}<0.001)$.

Conclusion: Consequently, we have observed that prednisolone administered immediately before the trauma can prevent the development of PTSD. Collectively a non-significant increase in the anxiety has been observed with metyrapon treatment, pharmacological use of glucocorticoids seems to be more important thant the endogenous steroids in the development of PTSD.

This research was supported by grants supplied from Marmara University Research Fund (SAG-C-YLP-141014-0347).

Keywords: metyrapone, elevated plus maze test, locus coruleus, noradrenalin, anxiety index

\section{P-052}

Event-related delta oscillations are sensitive to progression of mild cognitive impairment

Emek Savaş DD ${ }^{1}$, Çavuş̧oğlu B ${ }^{2}$, Güntekin B ${ }^{3}$, Ada E ${ }^{4}$, Başar $\mathrm{E}^{3}$, Yener $\mathrm{GG}^{5}$

'Department of Psychology, Dokuz Eylül University Faculty of Arts, Izmir, Turkey; 'Department of Neurosciences, Dokuz Eylül University Institute of Health Sciences, Izmir, Turkey; ${ }^{3}$ Brain Dynamics, Cognition and Complex Systems Research Center, Istanbul Kultur University, Istanbul, Turkey; ${ }^{4}$ Department of Radiology, Dokuz Eylül University Medical School, Izmir, Turkey; ${ }^{5}$ Department of Neurology, Dokuz Eylül University Medical School, Izmir, Turkey

Objective: Mild cognitive impairment (MCI) is considered as prodromal stage of Alzheimer's disease (AD). Decreased delta event-related oscillations (EROs) due to cognitive impairment have been demonstrated in prior studies of $\mathrm{AD}$ and MCI. In this study, we aimed to define an electrophysiological biomarker to predict the conversion from MCI to AD by using delta EROs and volumetric magnetic resonance imaging (vMRI). 
Methods: Twenty-two MCI patients and 23 matched healthy controls were participated. EEG was recorded from F3, Fz, F4, $\mathrm{C} 3, \mathrm{Cz}, \mathrm{C} 4, \mathrm{P} 3, \mathrm{Pz}, \mathrm{P} 4, \mathrm{O} 1, \mathrm{Oz}$ and $\mathrm{O} 2$ using classical auditory oddball paradigm. EEG and MRI were repeated after one year follow-up. The delta $(0.5-3.5 \mathrm{~Hz})$ EROs and volumes of whole brain, right and left hippocampi of stable and progressive MCI at baseline and follow-up were compared.

Results: Delta ERO were lower in MCI at baseline and followup $[\mathrm{F} 1,43=11.394, \mathrm{p}<0.003]$. Progressive MCI had lower delta ERO than stable MCI at F3 electrode site at baseline. vMRI revealed that MCI had lower volumes at baseline in whole brain and left hippocampus, and at follow-up in whole brain, right and left hippocampi. Moreover, a moderate correlation was found between left hippocampus volumes and delta ERO at C4 electrode site.

Conclusion: These findings demonstrate that delta ERO responses may detect early changes in MCI and indicate the patients who will progress to $\mathrm{AD}$ in advance. Brain oscillatory responses have the potential to be investigated as a candidate biomarker in MCI/AD. This study was supported by TUBITAK 112S459 grant.

Keywords: mild cognitive impairment, Alzheimer's disease, event-related oscillations, delta, volumetric MRI

\section{P-053}

\section{Investigation of visual P300 responses and neuropsychological tests in Parkinson's disease patients}

Özmüş $\mathrm{G}^{1}$, Emek Savaş DD ${ }^{2}$, Yerlikaya $\mathrm{D}^{1}$, Çakmur R ${ }^{3}$, Dönmez Çolakoğlu B $B^{3}$, Yener $\mathrm{GG}^{3}$

'Department of Neurosciences, Dokuz Eylül University, Izmir, Turkey; ${ }^{2}$ Department of Psychology, Dokuz Eylül University Faculty of Arts, Izmir, Turkey; ${ }^{3}$ Department of Neurology, Dokuz Eylül University Medical School, Izmir, Turkey

Objective: Cognitive changes develop in Parkinson's disease (PD) in addition to cardinal motor symptoms. Event-related potential (ERP) is a non-invasive and low-cost tool which measures electrical brain activity during cognitive process. P300 is associated with focused attention, working memory, signal detection and decision making processes. In this study, visual P300 responses and their relation to neuropsychological test (NPT) scores in PD patients and healthy controls were investigated.

Methods: Eighteen PD patients and 18 matched healthy controls were participated. Detailed NPT were applied to all participants. EEG were recorded from F3, Fz, F4, C3, Cz, C4, P3, $\mathrm{Pz}, \mathrm{P} 4, \mathrm{O} 1, \mathrm{Oz}, \mathrm{O} 2$ locations using classical visual oddball paradigm. Amplitude and latency values of P300 responses were measured. Group comparisons were carried out with repeated measures of ANOVA and correlations between P300 responses and NPT scores were performed by partial correlation tests taking age, gender and education as covariates.
Results: Significant differences were found between groups on P300 amplitudes $[\mathrm{F}(1,31)=9.265 ; \mathrm{p}=0.005]$. P300 amplitudes were significantly lower in $\mathrm{PD}$ patients at $\mathrm{F} 3, \mathrm{FZ}, \mathrm{Cz}, \mathrm{C} 4, \mathrm{Pz}$ and $\mathrm{P} 4$ electrode sites than healthy controls. P300 latency and NPT scores did not differ between groups. Moderate correlations were found between P300 amplitudes and Stroop test, digit span forward and lexical fluency.

Conclusion: Visual P300 amplitudes were significantly lower in PD patients than healthy controls and found to be associated with NPT scores. Decreased P300 amplitudes in PD possibly reflect disease related subclinical cognitive changes which may not be captured yet by neuropsychological assessment.

Keywords: Parkinson's disease, visual, P300, ERP, neuropsychological assessment

\section{P-054}

\section{Lucid dreaming in medical students}

$\underline{\mathrm{Ural} \mathrm{A}^{1}}$, Yokuşoğlu $\mathrm{C}^{2}$, Atasoy $\mathrm{M}^{1}$, Tekeli N${ }^{1}$, Ulus $C^{1}$, Emül $\mathrm{M}^{3}$

'Cerrahpaşa Faculty of Medicine, Istanbul University, Istanbul, Turkey; ${ }^{2}$ Department of Psychiatry, Istanbul University, Istanbul, Turkey; ${ }^{3}$ Department of Psychiatry, Istanbul University Cerrahpasa Medical Faculty, Istanbul, Turkey

Objective: Lucid dreaming is a phenomenon in which dreamers can be aware of dreaming and sleeping. We aimed to investigate the frequency of lucidity, metacognition and dream anxiety in medical students. The medical students may frequently face unfixed sleep/wake up time, stress and insomnia. We compare in terms of male and female medical students lucidity.

Methods: We chose a survey for lucidité, metacognition and dream anxiety. This survey of medical students have applied. There were 379 male and 537 female medical students in the sample. Lucidity testing, consisted of 6 sub-items. They were insight, control, thought, realism, memory and dissociation. We used the MCQ-30 test for metacognition. The MCQ-30 is comprised of five factors: positive beliefs about worry; negative beliefs about thoughts concerning uncontrollability and danger; cognitive confidence; need for control over thoughts; cognitive selfconsciousness. We use VDAS for dream anxiety. Statistical analysis was performed with SPSS-20.

Results: The mean total lucidity score was $56.22 \pm 15.46$ points according to $\mathrm{LuCiD}$ scale. There were significant differences between male and female medical students according to LuCiD sub items as: Control, Thought, Realism, Dissociation. The mean total metacognition score was $112.22 \pm 73.68$ points according to MCQ. The mean VDAS score was $57.00 \pm 23.61$ and dream anxiety was significantly higher in females than males. There were significant positive correlations between all scores of total/sub items in LuCiD and total MCQ scores.

Conclusion: Lucidity is associated with metacognition and anxiety dream. Metacognition and dream anxiety was significantly higher in females than males.

Keywords: lucid, dream, medical, student, gender 


\section{P-055}

Involvement of alpha-adrenergic receptors in the antidepressant-like effect of gallic acid

Turan N, Aydin H, Özkay DÜ1, Can ÖD, Öztürk Y

Department of Pharmacology, Anadolu University, Eskişehir, Turkey

Objective: Central nervous system related activities of gallic acid (GA), 3,4,5-trihydroxybenzoic acid, have been described previously. Especially, antidepressant-like effect of this natural polyphenol has become more conspicuous in recent years. However, information related to pharmacological mechanisms underlying this effect is quite limited. Therefore, in this study, we planned to investigate possible contribution of adrenoceptors to the antidepressant-like effect of GA.

Methods: Adult BALB/c female mice, weighing 30-35 g, were used for the experiments. Antidepressant effect screening studies were carried out with tail suspension test (TST). Further, spontaneous locomotor activity was assessed by activity cage tests. Possible participation of alpha-adrenergic receptors to the pharmacological effect of GA was investigated using phentolamine (non-selective alpha-adrenoceptor antagonist) and probable contribution of beta-adrenoceptors was examined using propranolol (non-selective beta-adrenoceptors antagonist). The experimental protocol was approved by the Local Ethical Committee on Animal Experimentation of Anadolu University.

Results: Obtained data demonstrated that GA $(60 \mathrm{mg} / \mathrm{kg})$ induced a significant decrease in the immobility time of mice with respect to the control group, indicating the antidepressant-like activity of this compound. In the activity cage tests, GA did not induce a significant alteration in the total number of locomotor activity. This data pointed out that, immobility time reducing effect of GA in the TST was not accompanied by changes in the locomotor activity. The anti-immobility effect was antagonized by phentolamine pre-treatment. However, GA induced decrease in the immobility time was not reversed by propranolol pre-treatment.

Conclusion: These results suggest that alpha-adrenoceptors, but not beta-adrenoceptors, are involved in the antidepressantlike effect of GA.

Keywords: gallic acid, antidepressant, adrenergic receptors, tail suspension

\section{P-056}

Evidence for the involvement of alpha-1-adrenoceptors in the antidepressant-like effect of quercetin

Alyu F, Can ÖD, Özkay ÜD, Öztürk Y

Department of Pharmacology, Anadolu University Faculty of Pharmacy, Eskişehir, Turkey

Objective: Preclinical findings indicated that quercetin has been shown to possess notable antidepressant-like effect with dual mechanism of action. In the present study we aimed to investigate possible involvement of alpha adrenoceptor subtypes in the antidepressant-like effect of this flavonoid.

Methods: Adult BALB/c female mice, weighing 30-35 g, were used for the experiments. Tail suspension test, which is a widely used behavioral despair model for antidepressant drug screening studies, was performed for this study. Spontaneous locomotor activities of the mice were assessed using the activity cage apparatus. Probable contribution of alpha adrenoceptor subtypes to the antidepressant-like effect of quercetin was investigated using prazosin (alpha-1-adrenoceptor antagonist) and yohimbine (alpha-2-adrenoceptor antagonist). The experimental protocol was approved by the Local Ethical Committee on Animal Experimentation of Anadolu University, Turkey.

Results: In the tail suspension test immobility time of mice was significantly decreased by quercetin treatment exhibiting the antidepressant-like effect of this flavonoid. Administration of quercetin did not change the total number of spontaneous locomotor activities indicating that the observed antidepressant-like effect was not affected from a probable change in the locomotor activity. The observed effect of this flavonoid was antagonized by prazosin pre-treatment. On the other hand, yohimbine pretreatment was not achieved to reverse shortened immobility time of quercetin-treated mice.

Conclusion: Obtained data pointed out that alpha-1-adrenergic receptors but not alpha-2-adrenoceptors contributed the antidepressant-like effect of quercetin.

Keywords: quercetin, antidepressant, adrenoceptors, tail suspension

\section{P-057}

\section{The contribution of resveratrol to the antiepileptic effects of diazepam and gabapentin}

Kaygısız B ${ }^{1}$, Özatik Y², Özatik O³ Bayraktar D ${ }^{4}$, Teksoy Ö$^{5}$, Cengelli Ünel $C^{1}$, Erol $\mathrm{K}^{1}$

${ }^{1}$ Department of Pharmacology, Eskişehir Osmangazi University Faculty of Medicine, Eskişehir, Turkey; 'Department of Pharmacology, Ahi Evran University Faculty of Medicine, Kırşehir, Turkey; ${ }^{3}$ Department of Histology and Embryology, Ahi Evran University Faculty of Medicine, Kırşehir, Turkey; ${ }^{4}$ Department of Physiology, Eskişehir Osmangazi University, Faculty of Medicine, Eskişehir, Turkey; ${ }^{5}$ Derpartment of Biology, Eskişehir Osmangazi University Faculty of Arts and Sciences, Eskişehir, Turkey

Objective: Resveratrol (RES), polyphenolic compound, was reported to have protective effect against convulsions. The effects of resveratrol alone or in combination with low-dose antiepileptics against pentylenetetrazole (PTZ)-induced seizures and its effects in brain regions were investigated.

Methods: Mice were divided into 8 groups: Control, RES (75 $\mathrm{mg} / \mathrm{kg}$ ), diazepam (DZ) $0.01-0.2 \mathrm{mg} / \mathrm{kg}$, gabapentin (GBP) $10-$ $20 \mathrm{mg} / \mathrm{kg}, \mathrm{RES}+\mathrm{DZ} 0,01 \mathrm{mg} / \mathrm{kg}, \mathrm{RES}+\mathrm{GBP} 10 \mathrm{mg} / \mathrm{kg}$. RES was given p.o for 7 days. In combination groups, DZ and GBP were applied i.p $30 \mathrm{~min}$ after the last dose of RES. Mice were observed for $30 \mathrm{~min}$ and seizure severity, seizure existence and 
mortality rates were evaluated. Cerebellum, cortex, hippocampus were isolated for the histopathological evaluation of necrosis, cell death and hemorrhage.

Results: In control group, the rate of seizure existence was determined as $100 \%$ whereas in GBP20 and DZ0, $2 \mathrm{mg} / \mathrm{kg}$ groups it was $14.3 \%$ and $0 \%$ respectively. The rates were determined as $77.8 \%, 85.7 \%$ and $42.9 \%$ in RES, GBP 10 $\mathrm{mg} / \mathrm{kg}$ and DZ0.01 mg/kg groups respectively and as $71.4 \%$ and $57.1 \%$ for RES+GBP10 $\mathrm{mg} / \mathrm{kg}$ and RES+DZ0.01 mg/kg combination groups respectively. The seizure severity score was 0 in GBP2 $0 \mathrm{mg} / \mathrm{kg}$ and DZ0, $2 \mathrm{mg} / \mathrm{kg}$ groups. The scores in RES, GBP10 mg/kg and DZ0, $01 \mathrm{mg} / \mathrm{kg}$ groups or in combinations of RES with GBP and DZ were determined as 4 and 5. There was no significant difference between groups in terms of the mortality rates. Histopathological evaluations also supported the results.

Conclusion: GBP20 mg/kg and DZ0, $2 \mathrm{mg} / \mathrm{kg}$ significantly decreased the seizure severity and provided protection against PTZ-induced seizures. Any preventive effect or any reduction in seizure severity were not observed in RES alone or in combinations with subeffective doses of GBP and DZ.

Keywords: diazepam, gabapentin, pentylenetetrazole, resveratrol, seizure

\section{P-058}

Midkine expression in kainic acid-induced epileptic seizures in rats

Ethemoğlu MS ${ }^{1}$, Kaçar $M^{1}$, Şeker FB ${ }^{1}$, Yazıhan $N^{2}$, Eyuboğlu $\mathrm{S}^{1}$, Tuna $\mathrm{BG}^{3}$, Yilmaz $\mathrm{B}^{1}$

'Department of Physiology, Yeditepe University, Medical School, Istanbul, Turkey; 'Department of Physiopathology, Ankara University, Medical School, Ankara, Turkey; ${ }^{3}$ Department of Biophysics, Yeditepe University, Medical School, İstanbul, Turkey

Objective: Epilepsy is one of the most common neurological disorder which occurs as a result of sudden, abnormal and hypersyncronized discharges of neurons. Midkine $(\mathrm{MK})$ is a neurotrophic factor which is extensively expressed in embryonic nervous system. Especially in embryonic neurons MK stimulates axonal sprouting, surviving and migration and also involves in Alzheimer's disease, cerebral ischemia and Parkinson's disease. It has been shown that MK expression is increased after brain insult; however it is not known if it is an endogenous repairing mechanism preventing further damage. TNF- $\alpha$ is a proinflammatory cytokine which is expressed after epileptic seizures. The aim of the project is to investigate the expression levels of MK and TNF- $\alpha$ during acute and subacute phases of epileptic seizures induced by Kainic Acid (KA) in cortex and hippocampus.

Methods: Adult male Sprague/Dawley rats were used in this study. $0.4 \mathrm{mg} / 0.2 \mu \mathrm{KA}$ was injected to hippocampus to induce seizures. The seizures were observed by EEG and behaviorally. Single dose injection of kainate receptor agonist KA in hippocampus induces pathologies similar to human temporal lobe epilepsy. The project included 3 groups: 1 . Vehicle group, 2. KA-1 day (decapitation 1 day after KA injection), 3. KA-3 days (decapitation 3 days after KA injection). ELISA was used to determine MK and TNF- $\alpha$ expression levels, TUNEL and cresyl violet staining were used to determine damaged neurons.

Results: We found that MK expression levels increased in cortex of KA-1 day group $(\mathrm{p}<0.05)$ however turned back to its control level after 3 days (KA-3 group). The expression level of TNF- $\alpha$ level elevated in both cortex regions of KA-1 and KA3 days groups.

Conclusion: This project is suggested that the expression of MK may play an important role in the acute phase of physiopathological mechanism of KA induced epileptic seizures.

\section{P-059}

The role of $\mathrm{p} 2 \mathrm{X7}$ receptors after epilepsy in mice

Keleștemur T, Çă̆layan AB, Beker MC, Yalçın E, Öztürk G, Kılıç E

Department of Physiology, Istanbul Medipol University, Faculty of Medicine, Istanbul, Turkey

Objective: ATP-gated ion channels, $\mathrm{P} 2 \mathrm{X} 7$ receptors, are promising and new targets for the treatment of epileptic seizures. $\mathrm{P} 2 \mathrm{X} 7$ purinoreceptors $(\mathrm{P} 2 \mathrm{X} 7)$ are cationic-selective ion channels gated by extracellular ATP and expressed throughout the central nervous systems. However, the role of $\mathrm{P} 2 \mathrm{X} 7$ receptors in the development of epileptic seizures is not well established. Here, we aimed to examine whether P2X7 receptors and their modulation have an impact on the development of epileptic brain activity, cortical microcirculation, cell signaling.

Methods: Animals were treated with $\mathrm{P} 2 \mathrm{X} 7$ receptor agonist BzATP; antagonist BBG. In addition, we have treated animals with a free radical scavenger melatonin. Epileptic brain activity was induced by intra-cortical penicillin injection and brain electrical activity was evaluated by electroencephalography (EEG). The cortical microcirculation was measured by laser speckle measurement and expression of pro-apoptotic and antiapoptotic proteins were analyzed by Western blot.

Results: While BzATP treatment increased spike frequencies, BBG treatment decreased them significantly, that evaluated within 2 hours after induction of epilepsy. Furthermore, free radical scavenger melatonin reversed pro-epileptic activity of BzATP, which was associated with improved cortical blood circulation, decreased pro-apoptotic protein activities and increased anti-apoptotic protein activities. In addition, the combination of BBG with melatonin did not further decreased brain electrical activity. We have observed that the beneficial effect of melatonin is developed independent from its receptors.

Conclusion: Here we provide evidence that modulation of $\mathrm{P} 2 \mathrm{x} 7$ receptor improves brain epileptiform and pro-epileptic activity of these receptors are reversed by melatonin, which is independent from its membrane receptors. The robust anti-convulsant 
effect encourages proof-of-concept studies with $\mathrm{P} 2 \mathrm{x} 7$ receptor antagonists or melatonin in human epilepsy patients.

Keywords: brain epileptiform activity, P2x7 receptors, BBG, melatonin

\section{P-060}

\section{Applications of stem cells on spinal cord injuries}

\section{Ulukaya ${ }^{1}$, Candar $\mathrm{E}^{2}$, Kabakaş $\mathrm{KN}^{3}$}

'Department of Anesthesiology and Reanimation, Faculty of Medicine, Ege University, Izmir, Turkey; ${ }^{2}$ Faculty of Engineering, Ege University, Izmir, Turkey; ${ }^{3}$ Faculty of Science, Ege University, Izmir, Turkey

Objective: In the present day spinal cord injury's treatment is insufficient. Against damage, SC tissue's regeneration is not enough. Important work is done nowadays with stem cells to be transported to the cell regeneration area provided. In animal models, different types of stem cells can be obtained a certain level of recovery of damaged when being implanted. However, this improvement is not known yet what mechanism is responsible for literally. The transplanted stem cells; neurotrophic factors secretion, ensuring the remyelination of axons and repair maintained through mechanisms such as the regulation of inflammation have been shown.tem cell therapies in the treatment of spinal cord injury; were examined and evaluate in this study.

Methods: We have our poster of the different stem cell types in the spinal cord injury (ESC, MSC, IPS, NSC) features, the advantages of the use in the treatment and try experiments on animals have given place to the results obtained.In addition, we investigated 6 clinical studies have been published which used bone marrow -derived macrophages and monocyte-derived stem cells.

Results: what kind of an ideal source and determine whether stem cells transplantation outcome occurring structural and functional recovery mechanism should be solved exactly prior to the implementation of stem cell-based therapy in the clinical field.

Conclusion: what kind of an ideal source and determine whether stem cells transplantation outcome occurring structural and functional recovery mechanism should be solved exactly prior to the implementation of stem cell-based therapy in the clinical field.

Keywords: spinal cord injury, stem cell therapy, mesenchymal stem cells, embryonic stem cell, neural stem cells

\section{P-061}

The role of opioidergic system in Panax ginseng extract

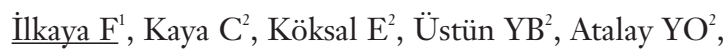
Gören $\mathrm{F}^{3}$, Guzel $\mathrm{H}^{1}$, Görüşük $\mathrm{S}^{4}, \mathrm{Ay} \mathrm{F}^{4}$

'Department of Pharmacology, Ondokuz Mayıs University, Samsun, Turkey; ' Department of Anesthesioogy and Reanimation, Ondokuz Mayıs University, Samsun, Turkey; ${ }^{3}$ Department of Internal MedicineGastroenterology, Van Training and Research Hospital, Van, Turkey; ${ }^{4}$ Class 3 Student, Ondokuz Mayıs University, Faculty of Medicine, Samsun, Turkey
Objective: Visceral pain is originated from visceral organs. Ginseng is a traditional plant which is a member of Panax class of Araliacae family. Its visceral analgesic effects have not been studied yet. We have researched the analgesic effect of Ginseng extract and opioidergic interaction on its analgesic effect on colorectal distension pain method.

Methods: Ginseng was dissolved in saline as $10 \mathrm{mg} / \mathrm{ml}$ and was given by intraperitonally. The threshold of pain was analysed by using colorectal distention induced visceral pain model. Experiments were performed on 54 male Sprague-Dawley rats weighing about 250-300 g. Experimental animals were divided into 9 groups; ginseng extract 25, 50, and $100 \mathrm{mg} / \mathrm{kg}$, morphine 0.5 , and $5 \mathrm{mg} / \mathrm{kg}$, naloxon $1 \mathrm{mg} / \mathrm{kg}+$ ginseng $100 \mathrm{mg} / \mathrm{kg}$, morphine $0.5 \mathrm{mg} / \mathrm{kg}+$ ginseng $25 \mathrm{mg} / \mathrm{kg}$, morphine $5 \mathrm{mg} / \mathrm{kg}+$ ginseng $100 \mathrm{mg} / \mathrm{kg}$ and saline were given to the groups one by one. Electrodes were embedded into external oblique muscles of rats. After a week, visceral pain was constituted by colorectal distetion (CRD) aparatus placed at colorectal region of each rat on experiment day. Viscero-motor response, which was originated from contraction of the muscles, was evaluated electromyographically before after drugs administration.

Results: Statistically, analgesia was observed only on experimental animals ginseng given at a dose of $100 \mathrm{mg} / \mathrm{kg}$ i.p. $(p<0.05)$. Naloxon has diminished the analgesic effect on Ginseng given at a dose of $100 \mathrm{mg} / \mathrm{kg}(\mathrm{p}<0.05)$. On combined groups of Morphine and ginseng neither synergism nor antagonism was observed.

Conclusion: Our results proved that Ginseng has an analgesic effect and this analgesic effect is reversed by naloxone.

Keywords: Panax ginseng, visceral pain, colorectal distension, opioid

\section{P-062}

\section{The effect of centrally injected arachidonic acid on respiratory system}

Erkan LG, Güvenç G, Altınbaş B, Yalçın M

Department of Physiology, Uludag University Faculty of Veterinary Medicine, Bursa, Turkey

Objective: Arachidonic acid (AA) is a polyunsaturated fatty acid that is present in the phospholipids of membranes of the body's cells, and is abundant in the brain. AA is involved in physiological and pathophysiological processes in the central nervous system. Recently we reported that AA leads to a pressor effect by activating brain thromboxane A2 (TXA2). The main object of the present study was to determine the effect of centrally injected AA on respiratory system and the mediation of central cyclooxygenase-TXA2 signaling pathway on AAinduced respiratory effects.

Methods: Experiments were performed in male Spraque Dawley rats. AA at doses of $0.25,0.5$ and $1 \mu \mathrm{mol}$ were injected intracerebroventricularly and respiratory rate, tidal volume and minute ventilation were recorded. To show mediation of the central cyclooxygenase-TXA2 signaling pathway in AA-evoked 
respiratory effects, pretreatment with nonselective cyclooxygenase inhibitor ibuprofen $(250 \mu \mathrm{g})$, or TXA2 synthesis inhibitor furegrelate $(250 \mu \mathrm{g})$ was carried out $5 \mathrm{~min}$ before injection with AA $(0.5 \mu \mathrm{mol})$, and the respiratory parameters were recorded. $\mathrm{pO} 2$ and $\mathrm{pCO} 2$ pressure of the all animals were measured before and 10 min after AA injection.

Results: Centrally injected AA caused dose- and time-dependent increase in respiratory rates, tidal volume and minute ventilation of rats. Also higher doses of AA increased $\mathrm{pO} 2$ pressure Moreover, ibuprofen and furegrelate blocked AA-evoked respiratory effects.

Conclusion: I.c.v. administration of AA exerts hyperventilation effects by increasing pO2 pressure in rats. Moreover, according to our findings, central cyclooxygenase-TXA2 signaling pathway mediates AA-induced respiratory effects. This study was supported by TÜBİTAK (114O093).

Keywords: arachidonic acid, respiratory parameters, cyclooxygenase, thromboxane A2, intracerebroventricular

\section{P-063}

Central apelin-13 infusion Increases hypothalamic NPY mRNA and serum ghrelin levels in rats

Tekin $\mathrm{S}^{1}$, Çiğgremiş $\mathrm{Y}^{2}$, $\underline{\text { Sandal } \mathrm{S}^{1}}$

'Department of Physiology, Inonu University Medical Faculty, Malatya, Turkey; '2Department of Medical Biology and Genetics, Inonu University Medical Faculty, Malatya, Turkey

Objective: Apelin was identified as the endogenous ligand of the human orphan $G$ protein-coupled receptor APJ. The apelin receptor gene has also been determined in mice and rats. We previously reported that the icv infusion of apelin-13 increased body weight and food intake in rats. These observations suggested that apelin might play a role in the central control of food intake. Arcuate POMC/NPY neurons are among the principal regulators of feeding behavior. While the NPY system stimulates food intake and decreases energy expenditure, the activation of POMC neurons has an opposite effect in the system. We hypothesizes that apelin may regulate the POMC/NPY system.

Methods: In the study, 21 Wistar-Albino male rats were randomly divided into three different groups as control, 1 and 10 nmol apelin-13 ( $\mathrm{n}=7$, each group). The rats were intracerebroventriculary infused vehicle, 1 and $10 \mathrm{nmol}$ apelin-13 via alzet osmotic mini pumps. After seven days, the animals were decapitated. Then, blood and brain tissue samples were collected. Serum ghrelin levels were measured by ELISA. The NPY and POMC mRNA levels were determined using RTPCR.

Results: Intracerebroventricular infusion of high dose apelin13 resulted in significant increases both in serum ghrelin and NPY mRNA levels in hypothalamus $(\mathrm{p}<0.05)$. However, POMC mRNA levels in hypothalamus were not significantly different.
Conclusion: Our results show that apelin-13 may change feeding behavior by affecting hypothalamic process.

Keywords: apelin, ghrelin, NPY, POMC, hypothalamus

\section{P-064}

The efficacy of melatonin on improvement of Alzheimer's disease in STZ-Induced rats

\section{Demir $M^{1}$, $\underline{\text { Yilmaz U }}^{1}$, Çolak $C^{2}$, Sandal $S^{1}$ \\ 'Department of Physiology, Inonu University, Faculty of Medicine, Malatya, Turkey; ${ }^{2}$ Department of Biostatistics and Medical Informatics, Inonu University, Faculty of Medicine, Malatya, Turkey}

Objective: Alzheimer's disease $(\mathrm{AD})$ is a neurodegenerative disease and the most common form of dementia that leads to memory dysfunction. The present study was designed to determine the protective effects of melatonin in a rat model of intracerebroventricular streptozotocin (STZ)-induced AD.

Methods: In the present study, forty male Wistar-Albino rats (220-280 g) were randomly divided into four groups $(\mathrm{n}=10)$ : sham, STZ, STZ+melatonin and STZ+ pinealectomy. After 14 days of surgical operations and injections, Morris water maze (MWM) test was performed. The parameters of time and distance traveled to reach the platform and time spent in correct quadrant were evaluated using ethovision automation software.

Results: The time and distance traveled to reach the platform of STZ infused rats were significantly increased while time spent in correct quadrant was significantly reduced in comparison to sham group $(\mathrm{p}<0.05)$. In the STZ+melatonin group was not observed any significant differences compared to sham group; but in the same group, time and distance traveled to reach the platform were significantly shorter and time spent in correct quadrant was significantly longer than STZ infused group $(\mathrm{p}<0.05)$. The parameters of time and distance traveled to reach the platform in the STZ+pinealectomy group were changed similarly in STZ-infused group $(\mathrm{p}<0.05)$.

Conclusion: Our results suggested that while melatonin supplementation prevented to development of $\mathrm{AD}$, melatonin absence did not affect.

Keywords: Alzheimer's disease, melatonin, pinealectomy, Morris water maze

\section{P-065}

Effects of central apelin-13 infusion on the hypothalamic TRH mRNA and serum TSH levels in rats

Erden $\mathrm{Y}^{1}$, Tekin $\mathrm{S}^{1}$, Özyalın $\mathrm{F}^{2}$, Çiğremiş $\mathrm{Y}^{3}$, $\underline{\text { Sandal S }}$

'Department of Physiology, Inonu University Medical Faculty, Malatya, Turkey; '2Department of Biochemistry, Inonu University, Faculty of Medicine, Malatya, Turkey; ${ }^{3}$ Department of Medical Biology and Genetics, Inonu University Medical Faculty, Malatya, Turkey

Objective: Apelin is a peptide hormone known as the ligand for the G protein-coupled APJ receptor. Apelin and APJ medi- 
ate a wide range of physiological actions including regulation of energy metabolism, food intake and fluid homeostasis. In our previous study, we had shown that the intracerebroventricular infusion of apelin-13 decreased serum free T3 and T4 levels. These results suggest that apelin-13 may play a role in the hypothalamus-hypophysis-thyroid axis. This study was designed to determine changes in the hypothalamic TRH mRNA and serum TSH levels after intracerebroventricular apelin-13 infusion.

Methods: In this study, 40 Sprague Dawley male rats were used, and were divided randomly into four groups $(n=10)$. The rats in the control group were not applied any surgical procedure. Sham and experiment groups received continually intracerebroventricular infusion via osmotic mini pump filled artificial cerebrospinal fluid or apelin-13 at concentration of 1 and $10 \mathrm{nmol}(10 \mathrm{ul} / \mathrm{h})$ for seven days. At the end of infusion, the rats were sacrificed and their brain and blood tissues were taken. TRH mRNA levels in hypothalamus were determined by RTPCR and serum TSH levels were measured by using ELISA.

Results: The levels of hypothalamic TRH mRNA were not different among the groups. While serum TSH levels significantly increased compared to control group $(\mathrm{p}<0.05)$, no difference was observed between sham and apelin-13 groups.

Conclusion: These results suggest that intracerebroventricular apelin-13 infusion is not effect hypothalamus-hypophysisthyroid axis at the central level. This study was supported Inonu University BAP (2013/180).

Keywords: apelin-13, intracerebroventricular infusion, TRH, TSH, hypothalamus

\section{P-066}

Inhibitory actions of carvacrol on inflammed and healthy isolated distal colon of mice

\section{$\underline{\text { Aydin } \mathrm{S}^{1}}$, Cakmak A}

'Department of Pharmacology, Faculty of Pharmacy, Anadolu University, Eskisehir, Turkey; ${ }^{2}$ Department of Pharmacology, Erciyes University, Faculty of Medicine, Kayseri, Turkey

Objective: Carvacrol is a monoterpene widely found in nature specially in plants. Recently it was shown to be ligand for Transient Receptor Potential channels (TRP). Due to presence and functions are known at distal mouse colon, the aim of the study was to test carvacrol on inflammed and healty distal mice colon.

Methods: Commercially obtained carvacrol was diluted in DMSO. Inflammation of colon was achieved by the intrarectal application of 100 microliter TNBS. Healthy and inflammed distal colon segments were transferred to isolated organ bath system and dose response curves were obtained for carvacrol against acetylcholine contractions. $\mathrm{R}$ packages were used for all the calculations and graphics.

Results: Carvacrol was observed to inhibit healthy distal colons but this actions were abolished on inflammed tissues.
Conclusion: Inflammation impairs of calcium, TRP channels and contraction related mechanisms used by carvacrol.

Keywords: carvacrol, TRP, distal colon, inflammation

\section{P-067}

Effects of melatonin and normobaric oxygen treatment on reperfusion injury

Beker MC, Çă̆layan AB, Keleştemur T, Yalçın E, Çăglayan B, Kılıç E

Department of Physiology, Istanbul Medipol University, Faculty of Medicine, Istanbul, Turkey

Objective: In order to protect brain before development of irreversible injury, the penumbral oxygenation is the primary goal of current acute ischemic stroke treatment. However, hyperoxia treatment remains controversial due to risk of free radical generation and vasoconstriction. We hypothesized that melatonin may reverse the possible side effects of hyperoxia in case of its unfavorable side effect or promote the beneficial effect of hyperoxia on reperfusion injury after middle cerebral artery occlusion (MCAo) in mice.

Methods: We examined the effects of normobaric oxygen (NBO) (21-, 70-, or $100 \%$, by inhalation) alone or in combination with melatonin $(4 \mathrm{mg} / \mathrm{kg}$, i.p.), on cerebral microcirculation, IgG extravasation, DNA fragmentation, infarct volume, brain swelling, neurological score and the activation of signaling molecules following 30 or 90 min of MCAo in mice.

Results: Both hyperoxia and melatonin improved cerebral blood flow significantly in the ischemic- core and penumbra analyzed by laser speckle imaging, which was associated with reduced IgG extravasation, DNA fragmentation, infarct volume, brain swelling and neurological scores. Notably, melatonin's neuroprotective activities were even more pronounced as compared with hyperoxia treatment. Furthermore, hyperoxia had no effect on eNOS and anti-apoptotic Bcl-XL activities, while melatonin increased them to the significant level. In addition, iNOS and nNOS activities were increased by hyperoxia. Increased phosphorylation of survival kinase Akt and decreased pro-apoptotic Bax activity was also observed. ERK$1 / 2$ phosphorylation was decreased in the $100 \%$ NBO condition.

Keywords: hyperoxia, reperfusion injury, cerebral microcirculation, BBB permeability, NOS

\section{P-068}

Comparison of the effects of bupropion and varenicline on morphine induced physical dependence

$\underline{\text { Allahverdiyev }}^{1}$, Shahzadi A², Önal B2, Özyazgan $S^{2}$, Akkan $\mathrm{AG}^{2}$

'Department of Pharmacology, Yüzüncü Yıl University, Faculty of Pharmacy, Van, Turkey; 'Department of Medical Pharmacology, Istanbul University Cerrahpasa Medical Faculty, Istanbul, Turkey 
Objective: Sympathetic hyperactivity causes morphine withdrawal symptoms. Noradrenergic neurons firing (NNF) in locus coeruleus leads to hyperactivation. Bupropion lowers NNF in locus coeruleus. The GABAergic transmission and endogenous opioids levels decrease during withdrawal. Varenicline prevents this decrease. Withdrawal leads to low dopamine levels in brain. Bupropion and varenicline increase dopamine levels. This leads to the hypothesis that bupropion and varenicline can be useful in morphine addiction.

Methods: Study involved 260-320g adult male Wistar albino rats. Morphine twice (08:00-20:00) daily (1st day 10, 2nd day $20,3 \mathrm{rd}$ day 30 , 4 th day 40,5 th day $50 \mathrm{mg} / \mathrm{kg}$, i.p.) was given for 5 days to rats. To elicit withdrawal symptoms, naloxone (2 $\mathrm{mg} / \mathrm{kg}$, s.c) was given on 6thday and monitored for 15 minutes. 15 minutes prior to naloxone bupropion $(5,10,20 \mathrm{mg} / \mathrm{kg}$, i.p.) or varenicline $(0.5,1,2 \mathrm{mg} / \mathrm{kg}$, s.c) were administered.

Results: Morphine and morphine+bupropion $(10,20 \mathrm{mg} / \mathrm{kg}$ ) showed significant reduction of withdrawal symptoms: jumping $(\mathrm{p}<0.05 ; \mathrm{p}<0.01$ respectively), wet- $\operatorname{dog}$ shake (WDS) $(\mathrm{p}<0.01 ; \mathrm{p}<0.001$ respectively), weight loss $(10 \mathrm{mg} / \mathrm{kg} ; \mathrm{p}<0.01)$ and total withdrawal score (TWS) $(20 \mathrm{mg} / \mathrm{kg}$; $\mathrm{p}<0.05)$ than that of morphine. Similarly, morphine and morphine+varenicline showed jumping $(0.5,2 \mathrm{mg} / \mathrm{kg} ; \mathrm{p}<0.05 ; \mathrm{p}<0.01$ respectively), WDS $(2 \mathrm{mg} / \mathrm{kg} ; \mathrm{p}<0.05)$, TWS $(0.5,1 \mathrm{mg} / \mathrm{kg} ; \mathrm{p}<0.05)$ significantly lower than morphine group. Weight loss was non-significant in all varenicline group $(\mathrm{p}>0.05)$.

Conclusion: As a conclusion, bupropion was found to be stronger on some withdrawal symptoms than varenicline. According to obtained results, it is considered that bupropion and varenicline can be useful and efficient in the treatment of morphine and other opioid addiction.

Keywords: bupropion, varenicline, morphine, naloxone, dependence

\section{P-069}

\section{Investigation of the recovery of the effects of methylphenidate on $\mathrm{D} 2$ receptors in rat cerebellum}

$\underline{\text { Bahcelioglu } \mathrm{M}^{1}}$, Take Kaplanoglu $\mathrm{G}^{2}$, Gözil R${ }^{1}$, Buru E${ }^{1}$, Helvacioğlu $\mathrm{F}^{3}$, Saraç GN ${ }^{2}$, Çalgüner E $\mathrm{E}^{1}$, Erdoğan $\mathrm{D}^{2}$

'Department of Anatomy, Gazi University, School of Medicine, Ankara, Turkey; '2Department of Histology and Embryology, Gazi University, School of Medicine, Ankara; ${ }^{3}$ Department of Histology and Embryology, Başkent University, School of Medicine, Ankara, Turkey

Objective: Methylphenidate is widely used in treatment of children with ADHD. Our aim was to determine effect of methylphenidate in rat cerebellum after several months following termination of drug administration.

Methods: 36 female Wistar albino rats were divided into two different dose (A1-10 mg/kg, A2-20 mg/kg) and time group (B1-three months drug, B2-three more follow-up months) with controls $(\mathrm{A} 0, \mathrm{~B} 0)$. Pre-pubertal rats received oral MPH dis- solved in saline solution for 5 days/week during 3 months. At the end of the experiment, all groups cerebellum was removed with perfusion and stained with anti-dopamine-2R primary antibody for being evaluated by photo-light microscope.

Results: In A0, D2R immunoreactivity was observed in scarce purkinje cells and in few neurons of molecular and granular layers. In A1, most purkinje cells displayed moderate D2R immunoreactivity while few cells showed negative staining. Number of immunostained cell was increased in granular layer. In A2, strong widespread staining was observed overall cerebellum. In $\mathrm{BO}$, immunoreactivity was similar to A0 in purkinje cells but widespread in granular layer. In B1, purkinje cells displayed resemblance to $\mathrm{A} 1$ in molecular and granular layer neurons, immunostaining was similar to B0. In B2, immunostaining was decreased. Immunostaining in molecular layer neurons of this group was similar to B0 but the staining was strong in granular layer neurons.

Conclusion: We observed that the recovery of structural findings in cerebellum were dose-related. In normal dose treated group, dopamine-2R staining was reversed totally but especially in high doses, recovery wasn't possible in granular layer neurons.

Keywords: methyphenidate, cerebellum, D2R, dose related

\section{P-070}

\section{The effects of Gly-GIn dipeptide on ischemia- reperfusion injury in rat brain cortical slice}

Sevdar G, Gül Z, Büyükuysal RL, Çavun S

Department of Medical Pharmacology, Uludag University Faculty of Medicine, Bursa, Turkey

Objective: Glycyl-Glutamine dipeptide supplementation was shown that beneficial to myocardial preservation in severe burn rats by improving cardiocyte energy metabolism. The present study we aimed to investigate whether GG also has a protective effect on ischemia-reperfusion injury in rat brain slices, and if this effect occurs by a mechanism dependent to directly dipeptited molecule or its breakdown products glycine and glutamine.

Methods: Cortical slices prepared from female Sprague Dawley rats were placed into the plates containing $2 \mathrm{ml}$ oxygenated physiological medium for 60 minutes of preincubation. Then slices were subjected to oxygen-glucose deprivated (OGD) medium and then oxygenated physiological medium (reoxygenation; REO). Tested GG, glisin or glutamine was added into the medium during both of OGD and REO periods. At the end of REO, slices were stained with $0,5 \%$ TTC and staining densities were measured. Incubation medium collected after REO, was used for measurement of $\mathrm{LDH}$ leakage from the slices.

Results: Thirty minutes OGD-REO caused $63 \%$ decline in TTC staining and 98\% increase in LDH leakage. Glycine-glutamine, at high concentration $(1 \mathrm{mM})$, completely protected the 
slices against OGD-REO-induced alterations in both TTC staining and LDH leakage. While glutamine $(1 \mathrm{mM})$ also exerted similar effect, glycine $(1 \mathrm{mM})$ was failed. While OGD-induced $\mathrm{LDH}$ leakage decreased to its control level by GlycineGlutamine and also by Glysine, Glutamine couldn't reduce it.

Conclusion: We conclude that brain function was damaged significantly after ischemia reperfusion injury and Glycyl-glutamine dipeptide supplementation is beneficial to that injury. The researches on the mechanism of Gly-Gln are still going on.

Keywords: Gly-Gln, brain, slice, LDH, TTC

\section{P-071}

Changes in the neurons of rats fed with corn syrup and protective effects of lipoic acid

Cankara $\mathrm{FN}^{1}$, Özmen $\mathrm{O}^{2}$, Yorgancıgil E ${ }^{3}$, Aşçı $\mathrm{S}^{4}$, Hançer Aydemir $\mathrm{D}^{5}$

'Departmant of Pharmacology, Süleyman Demirel University, Isparta, Turkey; ${ }^{2}$ Department of Pathology, Mehmet Akif Ersoy University, Burdur, Turkey; ${ }^{3} 6$ th Grade Medicine Student, Suleyman Demirel University, Isparta, Turkey; ${ }^{4}$ Department of Neurology, Isparta Gulkent State Hospital, Isparta, Turkey; ${ }^{5}$ Department of Medical Services and Techniques, Division of Medical Services and Techniques, Vocational School of Health Services, Suleyman Demirel University, Isparta, Turkey

Objective: Excess consumption of fructose contained in highfructose corn syrup (HFCS) can promote dementia pathogenesis through increased central neuronal insulin resistance and deposition of beta amyloid (associated with Alzheimer's disease) also through apoptosis. The present study considers the protective effects of alpha lipoic acid (LA) on brain cortex and hippocampus damages induced by chronic HFCS consumption.

Methods: The female rats were randomly divided into three groups ( $\mathrm{n}=24)$; Control group (C) (only given standard commercial diet and tap water); HFCS group (30\% of F30 HFCS solution was given during 10 weeks); HFCS+LA group (30\% of F30 HFCS solution was given during 10 weeks and $100 \mathrm{mg} / \mathrm{kg}$ LA was given orally for last 6 weeks of the experiment). Hippocampus and brain cortex were removed for histopathological and immunohistochemical (Caspase-3) examinations.

Results: No gross findings were observed in any group. Histopathology revealed slight hyperemia and edema in brains belonging HFCS group. Slight numbers of degenerative changes in neurons in both hippocampus and brain cortex were seen in two rats from HFCS group. None of the rats belonging HFCS+LA and C groups were exhibited any histopathological changes in brains. Caspase- 3 immunohistochemistry of the brains revealed only slight number caspase- 3 positive cells in two rats from corn sugar group in brain cortex.

Conclusion: HFCS slightly increased number of degenerative neurons in brain cortex and hippocampus. In addition slight caspase-3 expression was observed in cortical neurons. This study showed that HFCS can cause slight histopathological changes in brain. But LA can prevent the lesions.

Keywords: high fructose corn syrup, alpha lipoic acid, brain cortex, hippocampus, caspase-3

\section{P-072}

Inflammation markers fetuin a and hsCRP levels in cerebral palsy patients

Unay-Demirel $\ddot{O}^{1}$, Güngör $\ddot{O}^{2}$, Yüksel $M^{3}$

'Department of Biochemistry, Bahcesehir University, Faculty of Medicine, Medical Park Göztepe Hospital, Istanbul, Turkey; '2Department of Physiotherapy and Rehabilitation, Bahcesehir University, Faculty of Health Sciences, Istanbul, Turkey; ${ }^{3}$ Department of Medical Laboratory Technicianship, Marmara University, Vocational School of Health Related Services, Istanbul, Turkey

Objective: Cerebral palsy is characterized by permanent but unchangeable movements, tonus and posture disorders resulting from non-progressive damage to the brain early in life. Hypoxia/ischeamia and inflammation are two major mechanisms for cerebral palsy in preterm infants. Fetuin A which is a glycoprotein is secreted by the liver. It is essential for mineral homeostasis and shows immunomodulatory functions. Serum fetuin A concentrations are highest during intrauterine life. Experimental studies have shown that fetuin A immunoreactivity in brain is reduced by age. In this study, we measured fetuin A and hsCRP levels in patients with cerebral palsy.

Methods: Children with cerebral palsy ( $n=34) 1-15$ years of age and healthy control subjects $(n=21)$ were included in the study. After overnight fasting, blood was collected, serum samples were separated. Fetuin A levels were measured during using enzyme linked immunosorbent assay (ELISA) and hsCRP in an automated analyzer. Results are given as mean \pm SEM and statistical analysis were performed, $\mathrm{p}<0.05$ is accepted as significant.

Results: Fetuin A levels in cerebral palsy patients were significantly higher than healthy children $(431.4 \pm 82.8 \mathrm{pg} / \mathrm{mL}$ vs. $151.9 \pm 64.8 \mathrm{pg} / \mathrm{mL} ; \mathrm{p}=0.029)$. But hsCRP measurements were not significantly changed in patients with cerebral palsy in comparison to the control group $(1.3 \pm 0.3 \mathrm{mg} / \mathrm{L}$ vs. $0.9 \pm 0.2$ $\mathrm{mg} / \mathrm{L} ; \mathrm{p}=0.467$ ).

Conclusion: Our results have shown that fetuin A levels are higher in cerebral palsy patients, which reflects the impact of the immunomodulatory and/or inflammatory effects.

Keywords: cerebral palsy, fetuin A, hsCRP, inflammation

\section{P-073}

\section{Morphologic effects of agomelatine on prefrontal} cortical neurons of diabetic rats

\author{
Söztutar $E^{1,2}$, Can ÖD ${ }^{3}$, Özkay $\ddot{U}^{3}$, Ulupınar $E^{1,2}$ \\ ${ }^{1}$ Interdisciplinary Neuroscience Department of Health Science Institute, \\ Eskisehir Osmangazi University, Eskişehir, Turkey; 'Department of \\ Anatomy, Faculty of Medicine, Eskisehir Osmangazi University, \\ Eskisehir, Turkey; ${ }^{3}$ Department of Pharmacology, Anadolu University, \\ Faculty of Pharmacy, Eskişehir, Turkey
}

Objective: Type II diabetes mellitus (DM) is a systemic disease that may also causes neuronal damage. Agomelatine, agonist of 
melatonine MT1 and MT2 receptors, is a nootropic and antidepressant drug producing adaptive neuronal changes. In this study, we aimed to investigate the effects of Agomelatine on neuronal changes in the prefrontal cortex of diabetic rats.

Methods: Sprague-Dawley rats were assigned to four groups: (1) Control group; (2) DM group induced by $45 \mathrm{mg} / \mathrm{kg}$ i.v. streptozotocin injection (3) A-40 group, treated with $40 \mathrm{mg} / \mathrm{kg}$ Agomelatine for 2 weeks after induction of DM, and (4) A-80 group treated with $80 \mathrm{mg} / \mathrm{kg}$ Agomelatine for 2 weeks after induction of DM ( $\mathrm{n}=6$ for each group). Following cardiac perfusion, brains were sectioned $(150 \mu \mathrm{m})$ and stained with GolgiCox method. Layer II/III pyramidal neurons in the prefrontal cortex were analyzed with Sholl method and apical spine densities were calculated.

Results: No significant difference was observed in the Sholl analyses of groups. There was a significant reduction in the spine density of DM group comparing to the controls. Negative effects of DM were recovered in both A-40 and A-80 groups and spine densities were found comparable to the control group. In spine type analyses, adverse effects of diabetes were observed predominantly on the mushroom type of spines. While stubby and thin types of spines were not affected from DM, thin type of spines showed a significant increase in A-80 group. These results suggest that Agomelatine might ameliorate the deleterious effects of diabetes in neurons of the prefrontal cortex.

Keywords: diabetes mellitus, Golgi-Cox, sholl, spine, prefrontal cortex

\section{P-074}

\section{Effects of huperzine-A on the toxicity of amiloid beta} in the brain and skeletal muscle cells

${\underline{\text { Türkseven } \mathrm{CH}^{1}}}^{1}$, Büyükakıllı B ${ }^{1}$, Ballı $\mathrm{E}^{2}$, Şahin $\mathrm{L}^{3}$, Pekoğlu $\mathrm{E}^{1}$, Yetkin $\mathrm{D}^{2}$

'Department of Biophysics, Mersin University Faculty of Medicine, Mersin, Turkey; ${ }^{2}$ Department of Histology and Embryology, Mersin University Faculty of Medicine, Mersin, Turkey; ${ }^{3}$ Department of Physiology, Mersin University Faculty of Medicine, Mersin, Turkey

Objective: It's planned to research whether inclusion-body myositis (IBM) which is very similar to $\mathrm{AD}$ pathophysiology and can develop on the muscle tissue of A, plaques in the advanced process of $\mathrm{AD}$ occur in the experimental $\mathrm{AD}$ model used in the study or not, to find out the effect of the model on cognitive functions and the therapeutics benefits of HuperzineA (Hup-A) on tissue damage as a result of A.

Methods: Sixty-eight healthy female Sprague-Dawley rats were included in this study. Rats were randomly divided into five groups. Ovariectomy following the D-galactose injection (i.p.) during 10 weeks, every day to $100 \mathrm{mg} / \mathrm{kg}$ was performed in rats to create an animal model of Alzheimer's.

Results: No statistically significant differences were observed in electromyography (EMG) activity of extensor digitorum longus (EDL). Measured parameter is occurred significant reduction between groups that biomechanical value is acquired by giving stimulation at single and different frequencies on EDL muscle. However, histological examination of muscle pathology was not observed findings of IBM pathology. It was considered that the decrease in the EDL muscle force was related by ovariectomy. As a result of study, the healing effect of Hup-A was determined on the Alzheimer's pathology.

Conclusion: In conclucion, the formation of IBM pathology needs to be improved $\mathrm{AD}$ model that is further aggravated. For this purpose, after the last injection of D-galactose, rats should be allowed to form their own aging or the execution time or the dose of D-galactose should be increased.

Keywords: Alzheimer's disease, extensor digitorum longus (EDL), brain, huperzine-A, EMG

\section{P-075}

\section{Effects of acute and chronic lithium treatment on electrophysiological and cognitive function}

Yousef $\mathrm{M}^{1}$, Kavraal Ş ${ }^{1}$, Bitiktaş $\mathrm{S}^{2}$, Artış AS ${ }^{3}$, Süer $\mathrm{C}^{1}$

'Department of Physiologyl Basic Medical Sciences, Erciyes University Faculty of Medicine, Kayseri, Turkey; ${ }^{2}$ Department of Physiologyl Basic Medical Sciences, Kafkas University Faculty of Medicine, Kars, Turkey; ${ }^{3}$ Department of Physiology/Basic Medical Sciences, Medeniyet University Faculty of Medicine, Istanbul, Turkey

Objective: Lithium $(\mathrm{Li})$ has a major impact in the prophylaxis and treatment of mania and bipolar disorder. Nitric oxide (NO)-cGMP pathway has been implicated in the induction of long-term potentiation (LTP) which is known to be the predominant mechanisms of learning process. Therefore we sought to determine the effects of lithium treatment on the Dentate Gyrus (DG)-LTP, and how it correlates with spatial learning and NO- producing cells in rats.

Methods: Field potentials were recorded form the DG, stimulating perforant pathways, in rats chronically $(20 \mathrm{mg} / \mathrm{kg}$ for 40 days) or acutely treated with $\mathrm{LiCO} 3$ and their corresponding control rats. In addition, performance of rats in a Morris water maze was measured to link behaviour of rats to electrophysiological findings. NO- producing cells determined by NADPHd histochemistry.

Results: $\mathrm{LiCO} 3$ infusion into the hippocampus resulted in enhanced LTP, especially in the late phases, but attenuated LTP was observed in rats chronically treated with lithium as compared to controls. Li-treated rats equally performed a spatial learning task, but did spend less time in target quadrant than saline-treated rats in Morris water maze, and showed anxiety-like behavior in open field area. The number of NO producing cells in animals treated with $\mathrm{Li}$ was significantly lower than that in the control group.

Conclusion: Consistent with preclinical literature supporting the neuroprotective effects of lithium, short term treatment can be associated with preservation of synaptic plasticity, but chronic treatment disrupts behavioural and electrophysiologi- 
cal indicators of hippocampal functions in vivo and decreases the number of NO producing cells.

Keywords: lithium, hippocampus, learning and memory, longterm potentiation, nitric oxide

\section{P-076}

Effects of maternal thyroxine treatment during lactation on learning and memory in adult rat progeny

Tan $B^{1}$, Bitiktaş $\mathrm{S}^{2}$, Özdoğan $\mathrm{K}^{1}$, Bakkaloğlu $\mathrm{U}^{1}$, Aşçıŏglu $M^{1}$, Serü $C^{1}$

'Department of Physiology/ Basic Medial Sciences, Erciyes University Faculty of Medicine, Kayseri, Turkey; ${ }^{2}$ Department of Physiology/ Basic Medial Sciences, Kafkas University Faculty of Medicine, Kars, Turkey

Objective: The goal of the present study was to investigate whether thyroid hormone produces relatively stable lifelong changes during development that could influence or alter the adulthood behaviors of offspring.

Methods: Offsprings of the 24 pregnant rats were enrolled to the study. Maternal hyperthyroid group $(\mathrm{MH})$ consisted of male rat offspring from mothers who were treated with L-thyroxine $(0.2 \mathrm{mg} \mathrm{kg}-1$ body mass, $1 \mathrm{~mL})$ during lactation period. Rats treated with L-thyroxine from PND39 to PND60 were consisted of adult-onset hyperthyroid group (AOH). Euthyroid group (EU) consisted of male rat offspring from control mothers. Spatial learning performance was assessed in the Morris Water Maze ( $\mathrm{n}=12-16$ per group). Animal's blood was taken after the probe trial, serum thyroxine levels were measured by ELISA ( $n=6$ per group). Measurements were all done in rats aged 60-66 days.

Results: fT4 levels were significantly higher in $\mathrm{MH}$ rats $(3.15 \pm 0.11 \mathrm{ng} / \mathrm{dL})$ and $\mathrm{AOH}$ rats $(2.91 \pm 0.19 \mathrm{ng} / \mathrm{dL})$ than control rats $(1.69 \pm 0.34 \mathrm{ng} / \mathrm{dL})$. A one way ANOVA followed by LSD test revealed that the $\mathrm{MH}$ and $\mathrm{AOH}$ rats swum more distance to find the hidden platform than control rats $(\mathrm{Ps}<0.005)$. This result was accompanied with faster swimming speed and higher anxiety levels in $\mathrm{MH}$ group as compared to the control. There was no significant differences in spend time in the target quadrant in probe trial amongst groups.

Conclusion: These results suggest that perinatal excessiveness of thyroid hormone has longstanding effects on hippocampal function and may account for memory problems experienced by adolescents with congenital hyperthyroidism.

Keywords: thyroid hormone, learning and memory, hippocampus, lactation

\section{P-077}

Favoring LTD over potentiation in adult hypothyroidism is prevented by selenium supplementation

Bayar Y, Dursun N, Babur E, Bitiktaş S, Tan B, Süer C

Department of Physiology, Basic Medical Sciences, Erciyes University Faculty of Medicine, Kayseri, Turkey
Objective: The goal of this study was to evaluate whether sodium selenite could afford protection against the effects of hypothyroidism on LTP (long-term potentiation) and longterm depression (LTD).

Methods: Adult rats were randomly divided into one of the three groups, PTU, PTU+Se and control groups. Hypothyroidism was induced by 6-n-propyl-2-thiouracil at a concentration of $0.05 \%$. Rats in the PTU+Se group were fed 10 ppm Se supplemented diet. Field potential recordings were made from the dentate gyrus, with stimulation of the medial perforant path. High or low stimulus protocols were used to induce LTP or LTD. Animal's blood was taken after the recording, serum thyroxine levels were measured by ELISA).

Results: Both PTU group and PTU+Se group had suppressed levels of fT4 $(0.11 \pm 0.05 \mathrm{ng} / \mathrm{dL}$ and $0.67 \pm 0.10 \mathrm{ng} / \mathrm{dL}$, respectively) as compared to the control rats $(2.02 \pm 0.23 \mathrm{ng} / \mathrm{dL}$; Ps $<0.001)$. In response to HFS, hypothyroid rats, whether or not supplementation with Se, showed an attenuation in the early phase of LTP, but a protective effect of Se supplementation on the late phase of LTP was found. In addition, LFS protocol failed to induce LTD in control and PTU groups whereas a durable LTD was induced in PTU+Se group.

Conclusion: Our data provide evidence that adult-onset hypothyroidism results in an altered balance between LTP and LTD, leading to favoring LTD over LTP. This can be underlie memory problems experienced by adolescents with adult hypothyroidism and prevent dietary sodium selenite.

Keywords: selenium, hypothyroidism, long-term Potentiation, long-term depression, hippocampus

\section{P-078}

\section{Anticonvulsive effects of tributyltin in pentylentetra-} zole induced epileptic seizures

Dağlı Gül AS ${ }^{1}$, Arıhan $\mathrm{O}^{2}$, Tuncer $\mathrm{M}^{1}$, Er Pişkin $\mathrm{R}^{1}$, Fadıllıŏlu $\mathrm{E}^{1}$

'Department of Physiology, Hacettepe University Faculty of Medicine, Ankara, Turkey; 'Department of Physiology, Yüzüncü Yıl University Faculty of Medicine, Van, Turkey

Objective: Epilepsy is a common neurological disorder characterized by uncontrolled electrical activity in the brain. Genetics, febrile childhood diseases and exposure to chemicals may result in epilepsy. Tributlytin (TBT) is an organotin compound often taken into body through water and/or seafood. Different kinds of organotin compounds appear to have various effects as proconvulsive/anticonvulsive. Aim of this study was to evaluate the effects of TBT on epileptic seizures.

Methods: For each group six Swiss-Albino mice weighing 2535 gr was used. Pentylenetetrazol (PTZ) $(80 \mathrm{mg} / \mathrm{kg})$ constitutes generalized tonic-clonic seizures. Phenytoin $(10 \mathrm{mg} / \mathrm{kg})$ an antiepileptic drug was used for comparing the effect. For evaluating the pro/anticonvulsant effect of TBT, it was administered in 20, 40, $80 \mathrm{mg} / \mathrm{kg}$ (ip) doses. Groups were GI: PTZ; 
GII: PTZ+corn oil (TBT solvent); GIII: PTZ+phenytoin; GIV: PTZ+TBT (20 mg/kg); GV: PTZ+TBT (40 mg/kg); GVI: PTZ+TBT ( $80 \mathrm{mg} / \mathrm{kg})$. Experiments were recorded by camera and first clonus time following PTZ application, the number and time of tonic-clonic seizured animals and the time spent in the stupor were calculated as mean \pm standard deviation. Kruskal-Wallis, MannWhitney-U tests and Bonferonni correction were performed at statistical analysis and $\mathrm{p}<0.05$ was accepted for statistical significance.

Results: First time clonus length were found higher at TBT groups compared to other groups $(\mathrm{p}<0.05)$. In TBT groups, reduction in number of tonic-clonic seizured animals and shortening of the duration in stupor (seconds) were similar with the phenytoin group. Shortening of this time suggests a possible anticonvulsant effect of TBT.

Conclusion: TBT treated groups exerted anticonvulsive effect in PTZ-induced epileptic seizure model. Further studies are needed to understand anticonvulsive effects of TBT.

Keywords: anticonvulsant, epilepsy, pentylentetrazole, tributyltin

\section{P-079}

Central injection of uridine and its nucleotides increase blood pressure and blood glucose in rats

Ulus İH

Department of Pharmacology, Acibadem University Medical Faculty, Istanbul, Turkey

Objective: Recently we demonstrated that pyrimidinergic system exists in brain and uridine enhances pyrimidinergic activity. The aim of the present was to determine effects of uridine and its nucleotides on blood pressure and glucose level.

Methods: Female rats (250-300 g) were anesthetized with sevofluorane. The left carotid artery cannulated for blood pressure recording and the guide cannula placed into the right lateral ventricle for intracerebroventricular (icv) drug injection. Rats were allowed about 3 hours for recovery from anesthesia before experiments.

Results: Icv injection $1000 \mathrm{nmol}$ of uridine or uridine monophosphate (UMP) induced short-lasting (2-5 min) slight (5-15 $\mathrm{mm} \mathrm{Hg}$ ) increases in blood pressure. UMP, but not uridine, also increased blood glucose by about $10-20 \mathrm{mg} / \mathrm{dl}$ at this dose. Lower doses of uridine and UMP were ineffective and both drug caused convulsions at higher doses. Uridine diphosphate (UDP) and uridine triphosphate (UTP) increased blood pressure and glucose levels dose- and time-dependent manner after icv injection of 100-1000 nmol/rat. The increases in blood pressure and blood glucose were slight and short-lasting, while the increases after $1000 \mathrm{nmol} / \mathrm{rat}$ of UDP and UTP were about $30-40 \mathrm{~mm} \mathrm{Hg}$ in blood pressure and $60-80 \mathrm{mg} / \mathrm{dl}$ in blood glucose levels. The increases in blood pressure lasted within 10-15 min, while the rises in blood glucose were maintained for 45-60 min after icv administration of $1000 \mathrm{nmol} / \mathrm{rat}$ UDP or UTP.
Conclusion: These data show that activation of brain pyrimidinergic system increases blood pressure and blood glucose levels.

Keywords: uridine, blood pressure, blood glucose, pyrimidinergic system, UDP

\section{P-080}

Decompression surgery to reverse the peripheral diabetic polyneuropathy

Yıldıran G, Akdağ O, Selimoğlu MN, Karameşe M,

Tosun $Z$

Department of Plastic, Reconstructive and Aesthetic Surgery, Selcuk University Faculty of Medicine, Konya, Turkey

Objective: Peripheral diabetic neuropathy is one of the major complications of diabetes. Preventing the complications, reversing the neuropathy, block the neuropathy and reducing pain are the aims. So, surgical therapies can be applied besides medical therapy. We aimed to present decompression therapy for painful neuropathy symptoms.

Methods: Case 1 44-year-old male, who had peripheral diabetic polyneuropathy in his lower extremity. He was diabetic for 20 years, he was receiving insulin therapy. He didn't see benefit from medical therapies. Bilaterally; neurolysis was done for his nervus peroneus communis, peroneus profundus, tibialis, plantaris medialis, plantaris lateralis and calcaneus for decompression. Case 2 73-year-old male, who had peripheral diabetic polyneuropathy in his lower extremity. He was diabetic for 25 years, he was receiving oral antidiabetic and insulin therapy. He benefitted minimal from the medical therapy. Neurolysis was done for the same nerves for decompression of his right lower extremity.

Results: Symptoms are evaluated with EMG objectively and VAS (visual analog scale) subjectively Both patients had clinically recovery and reduction the VAS score in postoperative first month. Both patients had recovery in EMG in postoperative third month. In first case; motor nerve conduction velocity was slow, sensory response action potential amplitude was lower, F wave maximal conduction was slow, all of them were improved. In case 2; all of them were slow or absent, all of them were improved.

Conclusion: We believe the decompression surgery for lower extremity peripheral nerves is an effective and valid method for relieving the neuropathy pain and improving the sensory recovery.

Keywords: decompression, surgery, diabetic polyneuropathy

\section{P-081}

The dose-dependent effect of melatonin on hyperthermic febrile seizure parameters

Aydın L ${ }^{1}$, Gündoğan NÜ${ }^{1}$, Yazıcı AC

'Department of Physiology, Baskent University Faculty of Medicine, Ankara, Turkey; ${ }^{2}$ Department of Biostatistics, Baskent University Faculty of Medicine, Ankara, Turkey 
Objective: Different pathways such as GABAergic and nitric oxide pathways were put forward for anticonvulsant effect of melatonin. It has been also known that melatonin decreases the levels of interleukin-1beta (IL-1 $\beta$ ). We have supposed that different mechanisms causes differences in seizure parameters, and aimed to investigate changes in a febrile seizure (FS) model by using different doses of melatonin.

Methods: Sprague-Dawley male rat pups were divided into four groups. FS was induced by placing the animals in a water bath at $45^{\circ} \mathrm{C}$. The control group (CG) was administered vehicle; the study groups (SGs) were given $80-100-150 \mathrm{mg} / \mathrm{kg}$ melatonin, 15 min before seizure. Rectal temperature (RT) that triggered seizure, latency, seizure duration and scale were determined.

Results: The RTs of the CG and SGs were different at seizure onset $(\mathrm{p}<0.001)$. RT was the highest in $150 \mathrm{mg} / \mathrm{kg}$ SG compared to other SGs $(\mathrm{p}<0.001)$. Seizure latencies were longer in the SGs when compared to the CG $(\mathrm{p}<0.001)$. The latency in $150 \mathrm{mg} / \mathrm{kg}$ SG was shorter than other SGs $(\mathrm{p}<0.001)$. The SG and CG were different for seizure scales and durations $(\mathrm{p}<0.001)$.

Conclusion: Melatonin showed anticonvulsant effect at all doses studied. However, determining the lowest RT in 80 $\mathrm{mg} / \mathrm{kg}$ and the highest RT in $150 \mathrm{mg} / \mathrm{kg}$ SGs suggested a dosedependent, biphasic effect of melatonin on IL-1 $\beta$. In addition, a shorter latency in $150 \mathrm{mg} / \mathrm{kg}$ SG suggested triggering of other/additional anticonvulsant mechanisms when high-dose melatonin is used. Therefore, this study must be supported by molecular methods in the future.

Keywords: febrile seizure, melatonin, rectal temperature, mechanism of anticonvulsant effect

\section{P-082}

\section{Evaluation of attention deficit and hyperactivity disorder at rest by functional MRI}

Gengeç $S^{1}$, İçer $S^{1}$, Gümüş KZ $Z^{2}$, Doğanay $S^{3}$, Özmen $S^{4}$, Koç GG ${ }^{3}$, Öztop DB ${ }^{4}$

'Department of Biomedical Engineering, Erciyes University Faculty of Engineering, Kayseri, Turkey; '2Department of Biophysics, Erciyes University Faculty of Medicine, Kayseri, Turkey; ${ }^{3}$ Department of Radiology, Erciyes University Faculty of Medicine, Kayseri, Turkey; ${ }^{4}$ Department of Child and Adolescent Mental Health and Diseases, Erciyes University Faculty of Medicine, Kayseri, Turkey

Objective: Functional magnetic resonance imaging makes mapping of brain activation possible based on the changes in hemoglobin's oxygenation in the blood vessels. While the brain is not busy with anything (i.e. resting state), some networks are active. Default mode network is one of them and draws attention in functional magnetic resonance imaging studies. In this study, quantitative determination of changes occuring in default mode regions of the brain at rest in children with attention deficit and hyperactivity disorder (ADHD) was aimed.

Methods: Functional magnetic resonance imaging was performed for eight ADHD patients and 8 controls, age range (9-16) of both groups. First off all pre-processing steps were applied on the anatomical and functional images. After that statistical analysis was to compare both groups. Obtained results were evaluated.

Results: In ADHD group compared to the control group, the number of activated voxels were increased in the posterior cingulate cortex and the medial prefrontal cortex. They decreased in the bilateral inferior parietal lobe which are regions of default mode network.

Conclusion: Quantitative differences in the activation of brain's default mode network at rest in ADHD compared to the control group provide information about the neurological basis of the disease.

Keywords: resting-state magnetic resonance imaging, default mode networks, attention deficit and hyperactivity disorder

\section{P-083}

\section{Usefulness of pulsed radiofrequency treatment in stroke patients: a case series}

Apilioğulları $\mathrm{S}^{1}$, Albayrak Gezer $\dot{\mathrm{I}}^{2}$, Levendoğlu $\mathrm{F}^{2}$

${ }^{\prime}$ Department of Anesthesia and Intensive Care, Pain Clinic, Selcuk University Faculty of Medicine, Konya, Turkey; ${ }^{2}$ Department of Physical Medicine and Rehabilitation, Selcuk University Faculty of Medicine, Konya, Turkey

Objective: The prevalence of stroke ranges from 46.1 to 73.3 per 1000 population. Health-related quality of life after a stroke can be affected by decreasing in function such as difficulties with community ambulation. After the stroke, the provision of environmental stimuli increase and diversity is known to have positive effects on neuronal recovery. Radiofrequency is a therapeutic modality that has been used for years for diseases associated with pain. Pulsed radiofrequency therapy (PRT) with neuromodulatory synaptic effects, regardless of the thermal effect, causes a change in the transmission. Cervical and lumbar radicular pain, complex regional pain syndrome in such cases, neuromodulation is applied in the complaint related dermatome using the PRT of the dermatomes of dorsal root ganglia (DRG). With this application cortex is stimulated by giving notice to the DRG and could contribute to neuroplasticity.

Methods: In this case series the results of the PRT application is submitted to the DRG for two patients suffered a stroke at different times while the treatment rehabilitation.

Results: There was significant improvement in sensory examination of the patient in 15 days, 24 hours. With improvement in sensory examination upper and lower limb functional use, the effectiveness of the rehabilitative treatment and patient compliance with exercise programs has been increased.

Conclusion: Accordingly, in stroke patients with sensory deficits DRG from the periphery to the PRT is stimulated and neuroplasticity can be accelerated by sending notice to the damaged cerebral regions. However, there is a need to randomized controlled trials for more definitive evidence.

Keywords: stroke, pulsed radiofrequency therapy, dorsal root ganglia 


\section{P-084}

Nanotechnological approaches of carbon nanomaterials for nerve regeneration

Bozokalfa G $^{1}$, Seydibeyoğlu MÖ², Özsöz EŞM ${ }^{3}$

${ }^{1}$ Nanotechnology Joint Doctoral Programme, Gediz University / Faculty of Natural Sciences, Izmir, Turkey; ${ }^{2}$ Material Science and Engineering Division, Izmir Katip Celebi Universityl Institute of Science, Izmir, Turkey; ${ }^{3}$ Nanotechnology Division, Gediz University, Institute of Science, Izmir, Turkey

Objective: Observe the effect of carbon nanomaterials (carbon nanotubes, carbon nanofibers, graphen) on neurone formation and regeneration in the biomedical field specially in neurobiology shed light on alternative medicine solutions.

Methods: Carbonnanomaterials has been processed by different methods such as chemical vapoir deposition and hummers which is the most common procedure for graphene and graphen oxides. Graphen particles are obtained by chemical and sonic processes of graphite layers. In chemical vapor deposition method using various gases 1 or 2-3 atom thick coatings are occured with high purity on copper, nickel bases. Graphens that are obtained by chemical vapor deposition used in electronic application, Hummers method also is essential for composite electrots and composite materials which has various characteristics and size. In addition to this application of carbon nanostructures has increaesed in biomedical applications as biomimetic scaffolds in recent years.

Results: Acording to histological and/or electrochemical analysis results the carbon nanomaterials can be applied effectively in neurobiology. The research shows carbon nano structures especially carbon nanotubes are widely used by the way the graphene studies are very limited.

Conclusion: In summary, the investigation of carbon nanomaterials (carbon nanotubes, carbon nanofibers, graphen) effect on neuron regeneration especially in biomedical field it is clear that nanotechnological approaches can be applied in neurobiology.

Keywords: carbon nanomaterials, neuron regeneration, carbon nanotubes, neurobiology, graphen

\section{P-085}

A cost-effective pupillometry system for characterizing task-evoked pupillary response

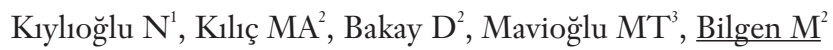

${ }^{1}$ Department of Neurology, Adnan Menderes University Faculty of Medicine, Aydın, Turkey; ${ }^{2}$ Department of Biophysics, Adnan Menderes University Faculty of Medicine, Aydın, Turkey; ${ }^{3}$ Department of Electronics and Automation, Adnan Menderes University Vocational School, Aydın, Turkey

Objective: Pupillometry has long been used for detecting subtle changes in pupil size following a cognitive load. But the current commercial systems are expensive to acquire. Thus, low cost alternatives are sought after for examining task-evoked pupillary response. Our aim was to construct a low-cost pupillometry system and demonstrate its merits with tests.
Methods: A web camera (INCA_IC-3562 model) was modified by removing its infrared filter and mounted on a chin rest. Light emitting diodes operating at infrared and visible spectrum were integrated for providing background and stimulus, respectively. The diodes were controlled by a microprocessor board (Arduino Uno). Stimulation was set as a periodic paradigm with variable frequency and duty cycle. Videos of pupil under task were acquired at 30 frames/second and processed using a software developed under Matlab platform. Temporal variations of the pupil area were detected and changes ' $n$ its timing and magnitude were analyzed.

Results: Preliminary results were provided to demonstrate the capability of the system. The data acquired under different stimulation paradigms indicated that the pupil area can accurately be measured from the video frames provided that reflections from both infrared and visible lights remain outside the pupil. At this time, equal duration on/off stimulus at $1 / 4 \mathrm{~Hz}$ appears to produce optimal pupil-response characteristics.

Conclusion: This system produces useful pupillometry data, but further development and implemetation are needed for potentially turning it into a low-cost alternative for studies involving autonomic nervous system, cognitive function, drug metabolism, pain responses, psychology, fatigue and sleep disorders.

Keywords: pupillometry, pupil, pupillary response, cognitive function

\section{P-086}

Real-time profiling the effects of quercetin and curcumin compunds on SHSY $5 Y$ cells

Dokumaci AH, Arslan AK, Yerer Aycan MB

Department of Pharmacology, Erciyes University Faculty of Pharmacy, Kayseri, Turkey

Objective: Curcuma longa is widely used for traditional treatment in Asia and India. It contains curcumin active ingredient which has antioxidant effect. Therefore this ingredient is investigated since it shows many effects in different fields. Antioxidant and anticancer effects of quercetin that extensively exists in many fruits have been shown in literature. In this study, real-time cell analysis of the effect of different concentrations of curcumin and quercetin on SHSY5Y neuroblastoma cells is aimed to be profiled with xCELLigence.

Methods: SHSY5Y cells were incubated in DMEM, \% 10 fetal bovine serum, $100 \mathrm{U} / \mathrm{ml}$ peniciline and $100 \mu \mathrm{g} / \mathrm{ml}$ streptomicyn, where the conditions are $370 \mathrm{C}$ and \% 5 CO2. Cells were dispensed so that 12500 cells/well. Xcelligence is a device that calculates cell index based on current and analyzing cells in real time. When the cell index approaches to 1 , quercetin and curcumin were added at the concentrations of $5,10,20,40,80 \mu \mathrm{M}$. The effect of quercetin and curcumin on cell index was analyzed by Xcelligence software.

Results: Quercetin and curcumin have shown dose dependent cytotoxic effect on SHSY5Y cells. The highest dose that is 80 $\mu M$ was determined as the concentration which decreases cell index at maximum rates. IC50 value of quercetin and curcum- 
in were respectively calculated as $115 \mu \mathrm{M}$ and $60 \mu \mathrm{M} 24$ hours after the treatment.

Conclusion: Quercetin and curcumin in a dose dependent cytotoxic effect on SHSY 5Y neuroblastoma cells are profiled with real-time cell analysis method for the first time and it has been found valuable to further investigation.

Keywords: xCELLigence, SHSY5Y, quercetin, curcumin

\section{P-087}

Chronically used agomelatine decreases gastrointestinal transit rate in rats

Can ÖD, Özkay ÜD, Üçel UI, Yıldız VC

Department of Pharmacology, Anadolu University Faculty of Pharmacy, Eskişehir, Turkey

Objective: Agomelatine is a new antidepressant drug with anxiolytic properties, acts through a unique mechanism of action, as a melatonin MT1 and MT2 receptor agonist and a serotonin 5-HT2C receptor antagonist. Pharmacological effect of this drug on melatonergic and serotonergic receptors may be hypothesized to gain this molecule a possible effect potency on gastrointestinal motility. Therefore, in this study we planned to examine probable alteration of gastrointestinal motility inducing by acute, short-term (6 weeks) and long-term (6 months) administrations of agomelatine $(40 \mathrm{mg} / \mathrm{kg})$ in rats.

Methods: Wistar male rats weighing 250-300 $\mathrm{g}$ were used for the experiments. Effect of agomelatine on gastrointestinal transit rate of rats was evaluated using the charcoal meal test. In this method, the migration distance of activated charcoal suspension from the pylorus to the most distal point of transit was measured. The percentage of "migration distance" was calculated and this value was used as a criterion for gastrointestinal transit rate.

Results: Obtained data indicated that acute agomelatine administration did not cause any significant alteration in the gastrointestinal transit rate of rats. On the other hand, percentage of migration distance was significantly lower not only in 6-weeks agomelatine administrated group but also in 6 months agomelatine treated group, than their respective control groups.

Conclusion: Findings of this study pointed out that long-term agomelatine treatment induces a significant retardation in the gastrointestinal motility of rats. This finding may have a clinical importance for a chronically used drug, like agomelatine.

Keywords: agomelatine, charcoal meal test, gastrointestinal transit rate

\section{P-088}

Effects of mianserin on hippocampal neuron numbers in streptozotocin-induced diabetic rats

Polat E ${ }^{1}$, Söztutar E ${ }^{1}$, Can ÖD², Özkay $\ddot{U}^{2}$, Ulupınar E ${ }^{1}$

${ }^{1}$ Interdisciplinary Neuroscience Department of Health Science Institute, Eskisehir Osmangazi University, Eskişehir, Turkey; '2Department of Pharmacology, Anadolu University Faculty of Pharmacy, Eskişehir, Turkey
Objective: Diabetes mellitus (DM) is one of the most common metabolic diseases in humans associated with adverse complications in many organ systems. Cerebral dysfunctions, including a high incidence of depression, are common findings in DM patients. The aim of this study was to investigate whether presynaptic $\cdot 2$ adrenergic receptor blocker Mianserin influences the neuronal number in the hippocampus of streptozotocin-diabetic rats.

Methods: Sprague-Dawley rats were divided into 3 groups $(\mathrm{n}=5)$; healthy controls, DM group received $45 \mathrm{mg} / \mathrm{kg}$, i.v. streptozotocin injection and treatment groups received Mianserin by oral gavage, $45 \mathrm{mg} / \mathrm{kg}$, per day for two weeks. Following intracardiac perfusion with $4 \%$ paraformaldehyde, serial sections were obtained from brains and stained with the Nissl method. Total number of granular and pyramidal cells from the dentate gyrus and CA1-3 regions of the hippocampus were estimated by optical fractionator probe of StereoInvestigator analysis system. Statistical comparisons of the data were done by using one-way analysis of variance test.

Results: Total numbers of pyramidal neurons in CA1-3 region were comparable in all groups. However, total numbers of granular cells in the dentate gyrus were significantly $(\mathrm{p}<0.01)$ lower in DM groups (9.02X105) than those of controls (17.8x105). Mianserin treatment did not significantly alter the total number of neurons $(8.382 \mathrm{X} 105)$ in diabetic rats.

Conclusion: These results suggest that a significant impairment occurring in neurogenesis in the hippocampal dentate gyrus might cause cognitive deficits in diabetic rats. Antidepressant activity of Mianserin was not effective in restoring this reduction in diabetic animals.

Keywords: streptozotocin, optical fractionator, hippocampus, antidepressant, Nissl staining

\section{P-089}

\section{The effect of memantine and NNC 55-0396} interaction on penicillin induced epileptiform activity

$\underline{\text { Alıcı SK}^{1}}$, Arslan G', Ayyıldız Mㅜ, Ağgar E

'Department of Physiology, Gaziosmanpasa University Faculty of Medicine, Tokat, Turkey; '2Department of Physiology, Cumhuriyet University Faculty of Medicine, Sivas, Turkey; 3Department of Physiology, Ondokuz Mayıs University Faculty of Medicine, Samsun, Turkey

Objective: Calcium ion plays an important role in the emergence of the epileptic activity. Memantine, which is an NMDAR antagonist is known as inhibit $\mathrm{Ca} 2+$ ions flows. The aim of this study is to investigate the effect of memantine and NNC 55-0396 interaction on the penicillin induced epileptiform activity.

Methods: Wistar rats were anesthetized with urethane (1.25 $\mathrm{g} / \mathrm{kg}$, i.p.) and placed in a rat stereotaxic apparatus. Bipolar two electrodes were placed over the somatomotor cortex of the left 
hemisphere and the ECoG activity was monitored on PowerLab. 500 IU Penicillin-G was injected intracortical (i.c.) to the rats in the control group. In the experimental groups; memantine $(5 \mathrm{mg} / \mathrm{kg}$, i.p) and T-type calcium channel blocker NNC 55-0396 (30 $\mu \mathrm{g}$, i.c) were administered separately 30 minutes after the application of penicillin. Also to investigate the interaction; memantine $(5 \mathrm{mg} / \mathrm{kg})+\mathrm{NNC} 55-0396(30 \mu \mathrm{g})$ were applied together 30 minutes after the application of penicillin. The ECoG activity were analysed off-line.

Results: The administration of memantine significantly decreased the mean of spike frequency in the 50th minutes and NNC 55-0396 decreased in the 40th minutes $(\mathrm{p}<0.05)$. When they were administered together the anticonvulsan effect appears earlier than they applied alone. The means of the spike frequency of the epileptiform activity were $104,09 \pm 4,51$ (control group) and 75.33 \pm 7.25 (memantine + NNC 55-0396 group) in the 20th minutes.

Conclusion: In accordance with the aquired data, it was concluded that using memantin and NNC 55-0396 together, they show their effects by using different mechanisms.

Keywords: epilepsy, memantine, NNC 55-0396, penicillin

\section{P-090}

\section{Comparison of hyperbaric oxygen versus iloprost in experimental central retinal artery occlusion}

Karaman $S^{1}$, Özkan B ${ }^{1}$, Furat Rençber $S^{2}$, Yazır $Y^{2}$, Yardımoğlu $\mathrm{M}^{2}$, Kara $\ddot{O}^{1}$, Altıntaş $\mathrm{O}^{1}$, Vural $\mathrm{Ç}^{3}$, Emek SK ${ }^{4}$, Kavram K

'Department of Ophtalmology, Kocaeli University Faculty of Medicine, Kocaeli, Turkey; '2Department of Histology-Embryology, Kocaeli University, Faculty of Medicine, Kocaeli, Turkey; ${ }^{3}$ Department of Medical Pathology, Kocaeli University Faculty of Medicine, Kocaeli, Turkey; ${ }^{4}$ Department of Underwater and Hyperbaric Medicine, Oksimed Hyperbaric Oxygen Therapy Center, Kocaeli, Turkey

Objective: Comparison of hyperbaric oxygen versus iloprost in rat models with experimental central retinal artery occlusion.

Methods: 28 healthy male Wistar albino rats were divided into four groups. The control group recieved no therapy or intervention. Sham, Iloprost and Hyperbaric oxygen (HBO) groups were resulted in central retinal artery occlusion. Serum physiologic was administered to sham group via intraperitoneal infusion for one day beginning from the reperfusion time. Besides, iloprost group also recieved intraperitoneal iloprost of 25 $\mathrm{ng} / \mathrm{kg} / \mathrm{min}$ for one day as well. In HBO group, 100\% oxygen therapy under $2.5 \mathrm{~atm}$ pressure was applied during $80 \mathrm{~min}$ for three times a day for the first two days followed by twice a day application for the next 7 days.

Results: As anticipated, apoptotic index was found lower with statistical significance in control group compared to other ones $(\mathrm{p}=0.000)$. HBO therapy was found more effective than sham group with statistical significance $(\mathrm{p}=0.000)$. Besides, iloprost therapy was also detected more effective when compared to sham group $(\mathrm{p}=0.000)$. However, no statistically significant difference was noted between hyperbaric oxygen and iloprost groups $(\mathrm{p}=0.514)$.

Conclusion: This is the pioneer study in which iloprost is evaluated in central retinal artery occlusion. It can be postulated that iloprost is as effective as hyperbaric oxygen therapy against occlusion of central retinal artery. Further studies with more animals and increasing doses of Iloprost covering combined application of iloprost and hyperbaric oxygen will be able to provide new aspects to the therapy.

Keywords: iloprost, retinal artery occlusion, apoptosis

\section{P-091}

Manuel asymmetry and motor performance distribution with sexual dimorphism foresight

Ölçgen B, Alpay Ş, Kazdağlı H, Sümbül S, Kutlu N

Department of Physiology, Celal Bayar University Faculty of Medicine, Manisa, Turkey

Objective: The aim was to investigate the distribution of motor activity by hand preference and gender.

Methods: Hand preference was obtainded with "Edinburgh handedness inventory". Motor skills of volunteers of Righthanded Female ( $n=33)$, Right-handed male $(n=31)$, LeftHanded Female ( $n=19)$, Left-Handed Male ( $n=17)$ was examined by International computer based Vienna Test System. Datas were evaluated with the SPSS 15.0 statistics programme.

Results: Left hand of left-handed females were statistically better than right hand of right-handed females by steadiness motor skill of hand and arm Right hand of right handed females were statistically better than right hand of right-handed males by Line Tracking Gross Motor Skill. Also Line Tracking results of right and left hand of Left-handed females were better than right and left hand of right-handed females. Eye hand coordination of left-handed males were statisticly beter than all other groups. Speed of hand-wrist in right-handed males was statisticly better than all other groups. Also Tapping results of right and left hand of left-handed females were better than right and left hand of right-handed females. Hand-finger fine motor skills of left-handed females were statisticly better than left hand of left-handed males.

Conclusion: Five different tests have showed that there was a significant correlation between hand preference value and fine, gross motor skills measured. We come to the conclusion on that motor control stability of Left-right hand skills and motor control of left-right hand can be asymmetric in brain and gender, hand preference, motor skills can effect one another statistically.

Keywords: hand preference, gender, motor skill, Vienna test system 


\section{P-092}

Association of polymorphisms in P2RX7 with Alzheimer's disease

Kortunay $\mathrm{S}^{1}$, Köseler $\mathrm{A}^{2}$, Tunç Ata $\mathrm{M}^{3}$, Öncel Ç $\mathrm{H}^{4}$, Sandıkçı $\mathrm{Y}^{5}$, Turgut $\mathrm{S}^{3}$

'Department of Pharmacology, Pamukkale University Faculty of Medicine, Denizli, Turkey; '2Department of Biophysics, Pamukkale University Faculty of Medicine, Denizli, Turkey; ${ }^{3}$ Medical Physiology, Pamukkale University Faculty of Medicine, Denizli, Turkey; ${ }^{4}$ Department of Neurology, Pamukkale University Faculty of Medicine, Denizli, Turkey; ${ }^{5}$ Department of Neurology, Denizli State Hospital, Denizli, Turkey

Objective: $\mathrm{P} 2 \mathrm{RX} 7$ purinergic receptor first cloned from a rat brain in 1996. This receptor is a non-selective cation channel and is widespread in the brain microglia. P2RX7 receptor is up-regulated in postmortem Alzheimer's Disease (AD) patients and also neurodegenerative animal models. This data support that $\mathrm{P} 2 \mathrm{RX} 7$ pathway involved in the progression of neurodegeneration. In our study, we investigated $\mathrm{P} 2 \mathrm{RX} 7$ receptor gene -1513 A / C polymorphism association with apoptotic processes mediated by inflammation, pain and AD (a neuroinflammation process).

Methods: Patients with similar epidemiology $(n=100)$ and control $(n=100)$ groups were parcipitated in this study. Genomic DNA extracted from peripheral blood samples of cases and controls was followed by RFLP.

Results: AD genotype frequencies for group AA and AC 69\%, $(\mathrm{CI})=62-75 ; 31 \%(\mathrm{CI}=24-37)$ respectively. A allele frequency was $84.5 \%$ for the $(\mathrm{CI}=79-89)$, and $\mathrm{C}$ allele was $\% 15.5$ (CI=10$20)$.In the control group, genotype frequencies were $36 \%$ $(\mathrm{CI}=29-42)$ for $\mathrm{AA}$ and $64 \%(\mathrm{CI}=57-70)$ for AC.A allele frequency was $68 \%(\mathrm{CI}=61-74), \mathrm{C}$ allele frequency was $32 \%$ (CI=25-38). $-1513 \mathrm{C}$ allele has been associated with $\mathrm{AD}$ sensitivity $(\mathrm{P}=0.0001$, odds ratio $(\mathrm{OR})$ for the wild-type allele $=2,565,95 \% \mathrm{CI}=1.539-4291)$. Protective effect for AD was determined for $1513 \mathrm{C}$ allele $(\mathrm{P}=0.0001, \mathrm{OR}=0.390$ for the mutant C allele, 95\% CI 0.233-0.650).

Conclusion: $\mathrm{P} 2 \mathrm{X} 7$ gene $1513 \mathrm{~A} / \mathrm{C}$ polymorphism is associated with $\mathrm{AD}$. with the / $\mathrm{C}$ polymorphism is present the. This data were reported for the first time in literature relationship in our study. This study was made with waste materials from PAUBAP project.

Keywords: Alzheimer's disease, P2RX7, genetic polymorphism

\section{P-093}

Protective effect of edaravone against manganeseinduced toxicity in cultured astrocytes

Evren $\mathrm{V}^{1}$, Apaydın $\mathrm{M}^{1}$, Khalilnezhad $\mathrm{A}^{1}$, Erbaş $\mathrm{O}^{2}$, $\underline{\text { Tașkıran }}^{1}$

'Department of Physiology, Ege University School of Medicine, Izmir, Turkey; 'Department of Physiology, Istanbul Science University, Istanbul, Turkey
Objective: Manganese (Mn), a trace metal, is essential for maintaining the normal regulation of many biochemical and cellular processes. However, accumulation of Mn due to excessive environmental exposure leads to neurological impairment, referred to as manganism. Edaravone (EDA), a potent free radical scavenger, has been shown to prevent brain edema after ischemia-reperfusion injury in animals. In the present study, we examined the protective effects of EDA against Mn toxicity in astrocyte cultures.

Methods: Astrocyte cultures were prepared from cerebral cortices of newborn Sprague-Dawley rats. Culture media were changed twice per week and the experiments were performed between 16 and 18 days. Astrocytes were treated in DMEM medium containing $\mathrm{Mn}(1 \mu \mathrm{M}, 10 \mu \mathrm{M}, 100 \mu \mathrm{M}$, and $1 \mathrm{mM})$ for 24 hours. Cells were pre-treated with different doses of EDA $(10 \mu \mathrm{M}, 100 \mu \mathrm{M}$ and $1 \mathrm{mM}) 6 \mathrm{~h}$ before $\mathrm{Mn}$ treatment. Cell viability (MTT), apoptotic cell death (Hoechst test) and lipid peroxide levels were evaluated in cultures. Statistical analysis was performed by one-way analysis of variance (ANOVA) and posthoc Bonferonni test.

Results: Our results showed that Mn significantly stimulated cell death in a dose-dependent manner in astrocyte cultures. The apoptotic cell death and lipid peroxides were significantly higher in Mn treated cultures. Treatment of astrocytes with EDA successfully suppressed oxidative stress and cell death due to Mn toxicity.

Conclusion: In conclusion, we propose that EDA may have beneficial effects against $\mathrm{Mn}$ toxicity. However, further studies are needed to elucidate the molecular mechanisms underlying protective effect of EDA.

Keywords: manganese, toxicity, astrocyte, edaravone, oxidative stress

\section{P-094}

\section{The effects of electromagnetic fields on learning and} memory in the pregnant rat

Cakır Z ${ }^{1}$, Bozoklu Akkar $\mathrm{O}^{2}$, Filiz $\mathrm{AK}^{3}$, Gültürk $\mathrm{S}^{3}$

'Department of Physiology, Gaziantep University, Gaziantep, Turkey, ${ }^{2}$ Department of Obstetrics and Gynecology, Cumhuriyet University Education and Research Hospital, Sivas, Turkey; ${ }^{3}$ Department of Physiology, Cumhuriyet University, Sivas, Turkey

Objective: It is reported that electromagnetic fields (EMF) increase cancer, behavioral changes, memory loss, Parkinson and Alzheimer disease. In this study, it's aimed to research the effect of EMA on learning and memory of pregnant WistarAlbino rats.

Methods: EMA was applied to 7-8 month old Wistar-Albino male, female and pregnant rats $(\mathrm{n}=96)$ as continuous / pulsed ( 1 , 5, 10 and $20 \mathrm{~h}$ ). In learning application, all groups are placed on light section of passive avoidance device (PAT). The time interval that rats enter dark section first time was evaluated as entry latency. EMA were applied Continuous groups as 1, 5, 10 and 20 
hours. EMA were applied pulsed group (according to $45 \mathrm{~min}$. exposed EMA and 15min. interval) as 1, 5, 10 and 20 hours. Rats were placed light section of PAT after 24 hours to evaluate the episodic memory. Electric shock application was evaluated whether rats remember or not. Freezing time in light section was accepted as episodic recall. Time interval values that rats passed from light to dark section was analyzed by using one-way anovatukey test.

Results: EMA application was a negative effect on memory in male and female rats.

Conclusion: Pregnant rats were less affected than other groups in continuous EMA application. Male rats were less affected than female rats in pulsed EMA application. The memory of pregnant rats wasn't affected by pulsed EMA applications. This result indicated that pregnancy have protective effect on brain. However, it should be supported by molecular and microscopic studies.

Keywords: electromagnetic fields, passive avoidance device, pregnant rat, learning and memory

\section{P-095}

Characterization of ERG channels in stallate neuron of the ventral cochlear nucleus

Yıldırım C, Bağcı C, Bal R

Department of Physiology, Gaziantep Üniversity Faculty of Medicine, Gaziantep, Turkey

Objective: ERG channels ( $\mathrm{kv} 11)$ is a member of voltagedependent potassium channel family, and has three sub-types called ERG1 (Kv11.1) ERG2 (Kv11.2 ) and ERG3 (Kv11.3). Although ERG channels are especially expressed in cerebellar purkinje cells, cochlear nucleus, facial nucleus, reticular nucleus, vestibular nucleus, their functions are not well known. The aim of the present study is to describe biophysical properties and physiological functions of ERG channel in cochlear nucleus.

Methods: Patch clamp tecnique was used for the characterization of ERG channel current. After 16-18 day-old mice were decapitated under anesthesia, the slices of $175 \mathrm{~mm}$-thick were cut by Vibratom then were incubated with pre-prepared and continuously oxygenated artificial cerebrospinal fluid at $33^{\circ} \mathrm{C}$. After these preparation intracellular recordings were taken from the neurons with whole-cell configuration. Terfenadine, an ERG channel blocker, was used to characterize ERG channels. The measures taken before and after ant-agonist application were evaluated with paired $\mathrm{T}$ test, and $\mathrm{P}<0.05$ was accepted as statistically significant.

Results: Resting membrane potential, were measured as $63.3 \pm 1.4 \mathrm{mV}$, respectively. In neurons with spontaneous activity, firing rate was significantly increased $(\mathrm{p}<0.001)$. In neurons with no spontaneous activity, firing rate induced by square current pulse was also significantly increased $(\mathrm{p}<0.001)$. Terfenadine significantly changed the resting membrane potential in a depolaring direction.
Conclusion: These findings obtained by this electrophysiological study indicate that ERG channels seems to exist in cochlear nucleus neurons.

Keywords: ERG channels, cohlear nucleus, patch clamp, electrophysiology

\section{P-096}

Morphological analysis of occipital condyles and foramen magnum as a guide for surgical approaches

Illhan $\mathrm{P}^{1}$, Kayhan $\mathrm{B}^{1}$, Ertürk $\mathrm{M}^{2}$, Şengül $\mathrm{G}^{2}$

'Department of Neuroscience, Ege University Institute of Health Sciences, Izmir, Turkey; '2Department of Anatomy, Ege University School of Medicine, Izmir, Turkey

Objective: Foramen magnum (FM) and occipital condyle (OC) area tumors are located in an anatomically complex region. Laterally extended open surgical approaches are preferred for treatment of FM and OC lesions compared to anterior and posterior approaches. Thus, certain parameters of FM and OC bony structures are critical for lateral surgical approaches.

Methods: Morphometric analysis was performed on 100 dried occipital bones. Measurements were made using a digital caliper accurate to $0,01 \mathrm{~mm}$ and a protractor. Six parameters were measured for FM and nine parameters for OC. Right-left differences were assessed using paired t-test. Foramen magnum shapes were also classified.

Results: The anteroposterior and transverse diameters of FM were $35.17 \pm 2.94 \mathrm{~mm}$ and $29.73 \pm 2.53 \mathrm{~mm}$, respectively. Anterior and posterior intercondylar distances were $22.46 \pm 2.98 \mathrm{~mm}$ and $41.54 \pm 3.77 \mathrm{~mm}$. Anteroposterior and transverse diameters of OC were $23.47 \pm 2.43 \mathrm{~mm}$ and $11.39 \pm 1.40 \mathrm{~mm}$, and sagittal intercondylar angle was $64.82 \pm 4.73$ degrees. OC anterior tip-opisthion distance was $39.43 \pm 3.33 \mathrm{~mm}$, OC anterior tip-basion distance $12.08 \pm 1.75 \mathrm{~mm}$, OC height $16.42 \pm 2.94 \mathrm{~mm}$ on the right and $15.67 \pm 2.63 \mathrm{~mm}$ on the left with a significant difference $(\mathrm{p}<0.05)$ between two sides. In the present study, we found 7 types of FM, the majority being tetragonal (24\%).

Conclusion: Typical approaches to FM are lateral extension of the posterior midline approach including the far lateral, and extreme lateral approaches, and the posterior midline approach. Transcondylar approach for lateral approach includes condylar drilling. This study presents a high number of morphometric parameters important for the lateral transcondylar approach and lateral approach to the FM.

Keywords: cranio-vertebral junction, foramen magnum, morphometry, occipital condyle

\section{P-097}

Hallmark of emissary vein in temporal bone with discussion on their evolutionary implications

Güleç Uyaroğlu F${ }^{1}$, Ertürk, $M^{2}$, Şengül $G^{2}$

'Clinic of Neurology, Izmir Tepecik Education and Research Hospital, Izmir, Turkey; 'Department of Anatomy, Ege University, School of Medicine, Izmir, Turkey 
Objective: The mastoid emissary vein (MEV) and its foramen have anthropological significance in transition to bipedalism and preferential intracranial venous flow into the vertebral plexus in upright man. The aim of our study was to explore the morphology, topography and morphometry of the mastoid foramen (MF) and internal opening of MEV (IOMEV).

Methods: The study included 200 adult (118 right and 82 left) human temporal bones of West Anatolian origin from the gross anatomy laboratory of Ege University, Department of Anatomy. Measurements were made using a caliper with 0.01 mm accuracy.

Results: The MF was observed single in 46 left (39\%) and 48 right (58\%) temporal bones, double in 45 left (\%38), 23 right (28\%), triple 17 left (14\%), 8 right (10\%) bones, quadruple 6 left $(5 \%)$. The mean diameter of MF was 2,04 (0,6-4,8) mm on left and 2,08 (0,5-6,2) $\mathrm{mm}$ on right side. IOMEV on the sigmoid sulcus was single in 55 left (47\%) and 56 right (\% 68) temporal bones, double in 45 left $(38 \%)$, right $19(23 \%)$, triple 14 left $(12 \%)$, right $6(7 \%)$. The foramen was absent in 4 left $(3 \%)$ and 1 right $(2 \%)$. The mean diameter of the IOMEV was 2,2 $(0,8-7,1) \mathrm{mm}$ on left and $2(0,6-5,3) \mathrm{mm}$ on right side.

Conclusion: Development of the intracerebral veins and their extracranial drainage is complex in humans. Adaptation of a bipedal hominid position was associated with anatomical and physiological changes in the venous system of the skull base to adapt to the concomitant changes in intracranial venous blood flow. The anatomy of these structures is important for the clinical presentation and treatment of complications such as thromboembolism and intracranial increased pressure syndrome.

Keywords: anatomy, antropology, mastoid emissary vein, mastoid foramen

\section{P-098}

\section{Alpha-tocopherol reduces spike-wave discharges in the WAG/Rij rat model of absence epilepsy}

\section{Gedikli Ö, Yildırım M}

Department of Physiology, Karadeniz Technical University Faculty of Medicine, Trabzon, Turkey

Objective: In this preliminary study, it is aimed to investigate the effects of alpha-tocopherol on the number and duration of spike-wave discharges (SWDs) in WAG/Rij rats, a genetic animal model of absence epilepsy.

Methods: Eight male WAG/Rij rats, 6 month old, were used in the experiments. Animals were implanted with a tripolar electrode for EEG recordings. Alpha-tocopherol was administered intramuscularly at a dose of $300 \mathrm{mg} / \mathrm{kg} / \mathrm{day}$ for 5 days. EEG recordings were made for $60 \mathrm{~min}$ before and $180 \mathrm{~min}$ after alpha-tocopherol injection (between 10:00-14:00 h). The number and duration of SWDs were statistically analyzed using the Wilcoxon test.

Results: When compared to baseline values, alpha-tocopherol administration decreased the number and total duration of
SWDs between 120 and $180 \mathrm{~min}$ after the first injection $(\mathrm{p}<0.05)$. It also decreased the number of SWDs between 60 and $120 \mathrm{~min}$ after the last doses $(\mathrm{p}<0.05)$. The number of SWDs was reduced by about $50 \%$, while the total duration of SWDs were reduced by about $70 \%$.

Conclusion: The present study demonstrates that alpha-tocopherol decrease the number and duration of SWDs in the WAG/Rij rats. These preliminary results suggest that alphatocopherol may be an effective adjuvant agent in the treatment of absence seizures.

Keywords: alpha-tocopherol, spike-wave discharge, absence epilepsy, WAG/Rij

\section{P-099}

Protective role of ascorbicacid against spike-wave discharges in a genetic model of absence epilepsy

Sekerci E, Gedikli Ö, Yıldırım M

Department of Physiology, Karadeniz Technical University Faculty of Medicine, Trabzon, Turkey

Objective: Ascorbic acid is a powerful water soluble antioxidant that reduces harmful oxidants in the central nervous system. The present study investigated the effects of ascorbic acid on the spike-wave discharges (SWDs) in freely moving WAG/Rij rats.

Methods: The experiments were carried out on 6-month-old male WAG/Rij rats $(n=8)$. EEG electrodes were chronically implanted under general anaesthesia. The animals were intraperitoneally injected ascorbic acid in the dose of 100 $\mathrm{mg} / \mathrm{kg}$. EEG recordings were performed using an electrophysiological data acquisition system (PowerLab 16/35) for four hours. Data analyzed using a paired samples t test.

Results: The $100 \mathrm{mg} / \mathrm{kg}$ ascorbic acid significantly decreased the number and duration of SWDs in the measured $120 \mathrm{~min}$ period when compared to baseline activity $(\mathrm{p}<0.001$ and $\mathrm{p}<0.01$ ). The number and duration of SWDs were reduced by about $50 \%$ compared with baseline values.

Conclusion: These preliminary data indicate that ascorbic acid has a protective effect against spontaneous SWDs in the WAG/Rij rat model of absence epilepsy.

Keywords: ascorbic acid, spike-wave discharge, absence epilepsy, WAG/Rij

\section{P-100}

Neuroprotective effect of chlorogenic acid on oxidative stress: a comparative study with resveratrol

Gül Z ${ }^{1}$, Bagdaş $D^{2}$, Büyükuysal RL ${ }^{1}$

'Department of Pharmacology, Uludag University, Faculty of Medicine, Bursa, Turkey; 'Experimental Animals Breeding and Research Center, Uludag University Faculty of Medicine, Bursa, Turkey

Objective: Since oxidative stress is a major component of harmful cascades activated in several neurodegenerative disor- 
ders, drugs having antioxidant properties are promising agents for new therapeutic strategies. Chlorogenic acid (CGA) is a natural phenolic compound with antioxidant properties. The present study was undertaken to compare the possible effects of CGA and its metabolites; caffeic acid, CA and quinic acid, QA with that of resveratrol on hydrogen peroxide (H2O2)-induced tissue damage in rat brain slices.

Methods: After $60 \mathrm{~min}$ of preincubation, cortical slices $(0.35$ $\mu \mathrm{m})$ were subjected to $200 \mu \mathrm{M}$ of $\mathrm{H} 2 \mathrm{O} 2$ for 1 hour. Tissue viability was evaluated by staining the brain slices with 2, 3, 5triphenyltetrazolium chloride (TTC). H2O2-induced alterations in malondialdehyde (MDA) and reactive oxygen species (ROS) levels were determined by 2,4-dinitrophenylhydrazine (DNPH ) and by 2,7-dichlorofluorescin diacetate (DCFHDA), respectively.

Results: While TTC staining was declined $48 \%$ by $\mathrm{H} 2 \mathrm{O} 2$ incubation, $57 \%$ and $50 \%$ increases were seen in MDA and ROS levels. Presence of CGA in the medium returned all the measured parameters to control levels. IC50 values of CA were calculated as 10, $3 \mu \mathrm{M}, 6,4 \mu \mathrm{M}$ and 3,7 $\mu \mathrm{M}$ for TTC staining, MDA and ROS, respectively. Although resveratrol exerted similar effects, its IC50 values were significantly less than that of CGA. In addition to these results it was also observed that $\mathrm{CA}$, one of the main metabolites of CGA, also has protective effects against $\mathrm{H} 2 \mathrm{O} 2$-induced alterations.

Conclusion: These results indicate that CGA may have therapeutic potential in counteracting oxidative damages in neurodegenerative disorders.

Keywords: chlorogenic acid, resveratrol, antioxidant, oxidative stress, neurodegeneration

\section{P-101}

Proteomic analysis of the brain extracellular matrix in the 5XFAD Alzheimer's mouse model

\section{Gürel B ${ }^{1}$, Sevinç C ${ }^{2}$, Cansev M², Baykal AT $^{3}$}

'Institute of Health Science, Istanbul Medipol University, Istanbul, Turkey; ${ }^{2}$ Department of Pharmacology, Uludag University, Faculty of Medicine, Bursa, Turkey; ${ }^{3}$ Department of Biochemistry, Istanbul Medipol University, Faculty of Medicine, Istanbul, Turkey

Objective: The extracellular matrix (ECM) molecules in the brain are produced by neurons, glia and non-neuronal cells and its importance in neurodegenerative disorders is being investigated. Recent studies revealed that some ECM molecules in the hippocampus have numerous effects on the synaptic responses and the stabilization of synaptic connectivity which are important for memory formation which is disrupted in Alzheimer's Disease (AD). The aim of our study is investigating the proteom profile of the hippocampal extracellular matrix in the early stages of $\mathrm{AD}$ to find whether there are early stage alterations in ECM compounds that can be linked to the genesis of neurodegeneration.

Methods: 5XFAD transgenic mouse models which carry five familial Alzheimer's Disease mutations were used as the exper- imental group $(n=6)$ and their non-transgenic littermates were used as the control group $(n=6)$. Morris water maze test was used to test memory impairment. The hippocampal extracellular matrix samples from each mice were obtained by intracranial cerebral microsidialysis push-pull method at month 3 . The changes in the protein expression profiles were eveluated by label-free nanoLC-MS/MS.

Results: 40 of 206 proteins that were identified in the samples by LC-MS/MS, show statistically meaningful expression difference between non-transgenic littermates and 5XFAD samples $(\mathrm{p}<0.05$ and min. fold change $>2)$.

Conclusion: According to Panther classification system, most of these 40 protein is related with catalytic activity $(64.7 \%)$ in terms of molecular function, metabolic $(41.8 \%)$ and cellular $(20 \%)$ processes in terms of biological processes.

Keywords: Alzheimer's disease, brain extracellular matrix, 5XFAD mouse model, proteomics, hippocampus

\section{P-102}

\section{Effects of rat amniotic membrane on nerve regeneration: a histological and stereological study}

Evirgen $\mathrm{O}^{1}, \underline{\text { Hayırl }_{1} \mathrm{EN}^{1}}$, Yılmaz ER $^{2}$, Hastürk AE

'Department of Histology and Embryology, Ankara University Faculty of Medicine, Ankara, Turkey; ${ }^{2}$ Unit of Neurosurgery, Dışkapı Yıldırım Beyazıt Education and Research Hospital, Ankara, Turkey; ${ }^{3}$ Unit of Neurosurgery, Dr. Abdurrahman Yurtaslan Ankara Onkoloji Education and Research Hospital, Ankara, Turkey

Objective: Repair of peripheral nerve injuries due to traumatic or iatrogenic factors the most important and successful method was microsurgery. Poor wound healing and fibrosis during regeneration impairs recovery and functionality as expected. New therapies are still continuing to develop for enhance nerve regeneration. In this study, we aimed to evaluate the effect of rat amniotic membrane (rAM) which was a biological material wrapped around primary epineurial anastomosis (PEA) region on wound healing and nerve regeneration.

Methods: Rats were randomly divided into 4groups control, transection injury, PEA after transection and PEA with rAM wrapping. 12 weeks after tissue samples collected from injury site and distal part fixed with $10 \%$ buffered formalin and $2.5 \%$ glutaraldehyde for light and electon microscopic examination. Paraffin sections stained with hematoxylin-eosin and semi-thin sections with toluidine-blue. Myelinated, unmyelinated axon numbers and myelin thickness was estimated using stereological methods.

Results: The histological evaluation appeared normal in control group. Examination of the transection injury group showed myelin degeneration, myelin ovoids, mast cells, Wallerian degeneration. There were a lot of Schwann cell nuclei and macrophages remowing myelin and axon debris. The endoneurial matrix enlarged due to fibroblastic and Schwann cell activity. PEA and PEA+rAM groups showed 
minifasicles formed by remyelinating axons. In the connective tissue sheaths a lot of mast cells detected. The thin myelinated axon numbers were more in PEA than PEA+rAM group.

Conclusion: As a biomaterial rAM wrapped around the nerve repair site have supportive effect on regeneration by providing an appropriate microenviroment and preventing fibrosis during regeneration.

Keywords: nerve regeneration, stereology, rat, amniotic membrane

\section{P-103}

\section{Melatonin is a protective effect of dopaminergic} neurons in a model of Parkinson's disease

$\underline{\text { Tanriöver } \mathrm{G}^{1}}$, Özsoy $\mathrm{O}^{2}$, Dilmaç $\mathrm{S}^{1}$, Kaya $\mathrm{Y}^{3}$, Aslan $\mathrm{M}^{4}$, Yıldırım $\mathrm{FB}^{3}$, A ğar A ${ }^{2}$

'Department of Histology and Embryology, Akdeniz University Faculty of Medicine, Antalya, Turkey; '2Department of Physiology, Akdeniz University Faculty of Medicine, Antalya, Turkey; ${ }^{3}$ Department of Anatomy, Akdeniz University Faculty of Medicine, Antalya, Turkey; ${ }^{4}$ Department of Biochemistry, Akdeniz University Faculty of Medicine, Antalya, Turkey

Objective: Parkinson's disease (PD) is characterized by progressive loss of dopaminergic neurons (DN) in the substantia nigra and depletion of dopamine in the corpus striatum. Melatonin is a potent free-radical scavenger and antioxidant. Its protective and anti-inflammatory effect in numerous neurological disorders. It is known to reduce detrimental effects of free radicals by stimulating antioxidant enzymes, however its role has not been studied in 6-hydroxydopamine (6-OHDA)induced rat model of PD. We aimed to elucidate the effects of melatonin on motor, Cox 2 activity determining of $\mathrm{DN}$ viability with tyrosine hydroxylase (TH) in these model.

Methods: Rats were divided into 5 groups: vehicle (V), melatonin treated $(\mathrm{M})$, parkinson $(\mathrm{P})$, melatonin treated+parkinson $(\mathrm{MP})$, parkinson+melatonin treated(PM). PD was created stereotactically via unilateral infusion of 6-OHDA into the medial forebrain bundle. Melatonin was injected at a single dose of $10 \mathrm{mg} / \mathrm{kg}$. Duration of melatonin teatment was 7 days for PM and 30 days for M, MP groups. PD was created on the 23rd day and melatonin treatment was continued for the remaining 7 days in the MP. PM group started receiving melatonin when PD was created and treatment was continued for 7 days.

Results: Locomotor activity decreased in $\mathrm{P}$ compared to $\mathrm{V}$ group, however melatonin treatment did not improve this impairment. 6-OHDA injection caused an obvious reduction in TH-positive DN viability as determined by immunohistochemistry. Caspase-3, Cox-2 activity confirmed to the results. Melatonin supplementation decreased DN death in PM, MP compared to $\mathrm{P}$ group.

Conclusion: Starting melatonin treatment before creating experimental PD was more effective on observed changes.

Keywords: Parkinson's disease, 6-hydroxydopamine, melatonin, tyrosine hydroxylase

\section{P-104}

EEG channel power spectrum analysis

Atl $\dot{\mathrm{I}}^{1}$, Akdeniz $\mathrm{G}^{2}$, Erdoğan $Z^{3}$

'Graduate School of Natural Sciences, YIldırım Beyazıt University, Ankara, Turkey; 'Faculty of Medicine / Department of Biophysics, Yıldırım Beyazıt University, Ankara, Turkey; ${ }^{3}$ Graduate School of Natural Sciences / Electronic and Communication Engineering, Yıldırım Beyazıt University, Ankara, Turkey

Objective: Electroencephalography (EEG) signals are known as bio-potential measured from the scalp surface or with the electrodes located in/on brain surface. Low amplitude signals of EEG data make it difficult to access information related to the area of the brain damaged for epilepsy patients. The accumulated power on the EEG recordings for each channel has a meaningful association with the seizures which may occur over time. The aim of this study is to calculate the power spectrum of alternating brain dynamics for each subject.

Methods: The EEG data used in this study is downloaded from web-site of epileptology department of Bonn University (http://epileptologie-bonn.de/cms/upload/workgroup/lehnertz/eegdata.html). For the calculations, we select the first channel of each EEG data set $\mathrm{O}$ and $\mathrm{Z}$ containing 10 healthy subjects, and set $\mathrm{F}$ and $\mathrm{S}$ containing 10 patients with epilepsy disorder. The data sets $\mathrm{O}-\mathrm{Z}$ include EEG recordings from scalp while data set $\mathrm{F}$ includes interictal and data set $\mathrm{S}$ include intracranial ictal EEG recordings. We have carried out fast Fourier transform (FFT) in Matlab for each individual's to compute power spectrum of EEG channel.

Results: As a result, the power spectrum of data set $\mathrm{S}$ which contains seizures generated larger values in contrast to other data sets. The values of the power spectrum were observed close to each other in healthy group data sets $(\mathrm{O}-\mathrm{Z})$.

Conclusion: These values provide information about recordings of impaired brain's electrical activity so that separation of EEG data including seizure can be distinguished effortlessly by using these findings.

Keywords: EEG, power spectrum, epilepsy, seizure

\section{P-105}

Gender specific analgesic actions of 1,4-cineole.

Cakmak A ${ }^{1}$, Aydın S

'Pharmacology, Erciyes University, Kayseri, Turkey; 'Pharmacology, Anadolu University, Eskisehir, Turkey

Objective: Monoterpenes are widely distributed in nature from primitive formss of life to higher vascular plants. 1.4-cineole is a member of this family and there very few experimental data on this compound. Due to high variation of 1.4-cineole results, the aim of the present studey was to test the analgesic actions of 1.4-cineol, on male and female mice.

Methods: 1.4-cineole, was commercially obtained and thermal algesic stimulus was used in all experiments and and cutoff time 
was $15 \mathrm{sec}$. Calculations of percent analgesic actions and graphics were performed by $\mathrm{R}$ programme.

Results: It was found that 1.4-cineole exerted analgesia only on males but not on female mice, suggesting the obligation to take into consideration of gender differences.

Conclusion: 1.4-cineole had gender specific analgesic actions and it was found that 1.4-cineole exerted analgesia only on males but not on female mice, suggesting the necessity to take into consideration of gender differences of monoterpenes. To the best of our knowledge, this is the first report on gender specific actions of 1.4-cineole and related compounds.

Keywords: monoterpene, TRP, 1.4-cineole, analgesia, gender specific action

\section{P-106}

Corrugator muscle compressions of the supratrochlear and supraorbital nerves for treatment of trigeminal cephalgia

Pınar Y, Govsa F, Ozer MA, Sirintürk S

Department of Anatomy, Faculty of Medicine, Ege University, Izmir

Objective: The contraction of the corrugator supercilii muscle (CSM) lead to supraorbital headaches appears as a result of negative emotions, such as anger, anxiety, fatigue, fear, and disapproval. Compression points of the supratrochlear nerve (St) and supraorbital nerve (So) in the (CSM) are important for treatment of cephalgia.

Methods: In fifty cadaver hemibrows, the St and So were investigated in their compression points and its relationships to CSM types.

Results: Three compression points of nerves (St/So) were identified as the entrance points into the frontal notch, the inside into the CSM, and the exit points of the nerve from the CSM. The five different CSM types were showed as rectangular shaped $(42.5 \%)$, three bellies (25\%), duplicate muscle $(12.5 \%)$, irregular flat (15\%) and hypoplastic types (5\%). During So and St's CSM is being under dense muscle mass, these affective nerves are sending a signal to the brain to create the headache. Appraising the relation between muscle and nerves, the CSM of the the rectangular shaped, three bellies and dublicate muscle types might be disadvantageous that these types may lead to major compression on the St and So. Irregular and hypoplastic types may be advantageous to treat with botox injection, because of these muscle types may results into minor compression.

Conclusion: Reporting different types of CSM were undefined before, entrapment of the nerves caused by the muscle could not be discussed in all ways. Defined topographical details of the So and St with CSM can help understanding to treat on supraorbital neuralgia of the individual pattern.

Keywords: supraorbital nerve, supratrochlear nerve, compression point, trigeminal cephalgia, peripheral nerve stimulation

\section{P-107}

Age-dependent effects of retigabin on genetic absance epileptic WAG/Rij rats

Karadenizli S, Şahin D

Kocaeli University Medical School, Physiology Department, Kocaeli, Turkey

Objective: Absance epilepsy is a type of non-convulsive epilepsy characterized by sudden loss of consciousness, bilateral synchronous and symmetrical EEG spike-wave complexes (SWD). Retigabine is an anticonvulsant drug used in the treatment of partial seizures and effects through the activation of M-type current dependent on KCNQ channels. The purpose of this study was to investigate the age dependent effects of Retigabine.

Methods: Recording electrodes were placed on skulls of 12 and 6-month WAG/Rij rats. Following the recovery period, baseline EGG recording were measured for 1 hour. After that, Retigabine $(5 \mathrm{mg} / \mathrm{kg}$ ) to experimental group and 20\% DMSO to control group were injected, intraperitoneally. EEG were recorded for 3 hour. Unpaired t test and non-parametric Bonferoni's test were used to compare changes between groups and changes in the group, respectively.

Results: After application of Retigabine to 12-month rats, the number and the total duration of SWD's in 1st and 2nd hour signifficantly increased compared to baseline $(\mathrm{p}<0.05)$. In the 6-month rats after application of Retigabine, there weren't signifficant changes in the number and duration of SWD's during 1st, 2 nd and 3 rd hours recordings ( $>0.05)$. The number and duration of SWD's in 12-month rats were signifficantly higher than 6-month rats in 1 st and 2 nd hour $(\mathrm{p}<0.05)$.

Conclusion: Application of Retigabine increased the number and duration of SWD's in aged group compared to young group. Increasing effect of Retigabine in aged rats may be related to facilitating effect on $\mathrm{K}+$ channel activity (similar to GABAB) modify T-type $\mathrm{Ca} 2$ + channels activity in the reticular thalamic nucleus.

Keywords: retigabine, absance epilepsy, $\mathrm{K}$ channel opener, spike-wave discharges

\section{P-108}

Effects of kisspeptin on locomotor acitivity and anxiety behavior in adult mice following intra-habenular infusion

\section{Eyuboğlu S, Yilmaz B}

Yeditepe University, School of Medicine, Department of Physiology, Istanbul, Turkey

Objective: Kisspeptin plays an important role in hypothalamic regulation of gonadotropin releasing hormone secretion. Specific receptor of this neuropeptide (GPR54) is expressed in brain areas in addition to the hypothalamus. Therefore, it is thought that kisspeptin may have effects other than its neuroendocrine control of reproductive functions. Lateral 
Habenula $(\mathrm{LHb})$ is a brain area where direct and indirect effects on dopaminergic and serotonergic systems are processed. In the present study, effects of kisspeptin thorough GPR54 receptors in the $\mathrm{LHb}$ on anxiety and motor activity behavior in adult mice were investigated.

Methods: Adult male Balb-C mice were divided into three groups ( $\mathrm{n}=3$ /group) as control, kisspeptin and kisspeptin antagonist (P234). The mice were placed in the stereotaxic frame under isofluoran anesthesia (2\%) and intra-habenular infusions were performed. The animals were observed to return normal feeding-drinking behavior within $30 \mathrm{~min}$ after anesthesia. Following the infusions, elevated plus maze and open field tests were performed to evaluate anxiety and locomotor activity, respectively. At the end of the experiments, all animals were decapitated. Brains were dissected out and stored at $-80^{\circ} \mathrm{C}$ for future analysis.

Results: In view of the preliminary findings, it was determined that stereotypic, ambulatory and horizontal locomotor movements were significantly reduced by $\mathrm{P} 234$ compared to the kisspeptin group $(\mathrm{p}<0.01, \mathrm{p}<0.05$ and $\mathrm{p}<0.01)$. In addition, distance parameter was significantly increased by kisspeptin in comparison to the P234 group and resting time in this antagonist group was significantly higher than the kisspeptin group $(\mathrm{p}<0.01)$. There was no significant change in the elevated plus maze findings among the groups.

Conclusion: It was observed that locomotor activity was increased by kisspeptin and decreased by kisspeptin antagonist following intra-habenular infusion. It is thought that motor activity inhibition processed by the dopaminergic system in the $\mathrm{LHb}$ is disinhibited by kisspeptin, and this inhibition is further increased by P234. New experiments involving brain microdialysis evaluation of dopaminergic neurotransmission in this model are planned.

Keywords: lateral habenula, kisspeptin, P234, GPR54, anxiety, locomotor activity, mice

\section{P-109}

Neuropsychometric profile aspects of REM sleep disorders among young migraine patients

Mantar $\mathrm{N}^{1}$, Çadırcı $\mathrm{F}^{1}$, Yılmaz Helvacı $\mathrm{N}^{2}$, Yamanoğlu $\mathrm{M}^{1}$, Toprak $\mathrm{G}^{1}$, Velioğlu HA ${ }^{1}$, Soğukkanlı Kadak K ${ }^{1}$, Hanoğlu L ${ }^{2}$

'Istanbul Medipol University, Faculty of Medicine, Department of Neuroscience, Istanbul, Turkey; ${ }^{2}$ Istanbul Medipol University, Faculty of Medicine, Department of Neurology, Istanbul, Turkey

Objective: Clinic aspects, cognitive patterns and results of form of REM sleep behavior disorder (RDB) among elder ages, are researched very well. In elder ages, idiopathic RBD characterize by low performance with executive visual and perceptual function is shown specific pattern with cognitive impairment. Recent studies show that these phenomenon are resulted with pathology of "synucleinopathy" degenerative states like Parkinson diseases, Lewy body dementia. However, (RBD)'s forms that are seen in young ages are studied very little and there is not any study about the cognitive profile of these phenomenon. Our aim in this study is to research if there is a similar cognitive effect between younger ages and older ages of a patient group with migraine.

Methods: In our study, we determined 11 RBD patients by questionnaire form among migraine patients from our previous related research. They were evaluated by recording a singlenight polysomnography. These patients and their 10 non-RBD controls who were selected by form as havin same demographic information with the patients were applied a neuropsychological test batter consists of verbal, nonverbal memory, executive and visual-perceptual functions.

Results: According to international polysomnography RBD criteria, six of the patients diagnosed RBD and five of them described motor enacting behavior. Both of groups were compared with 10 non-RBD control group patients in terms of neuropsychometric batteries test performance.

Conclusion: The results of our study will be discussed in the light of literature.

Keywords: REM sleep behavior disorder, cognition, neuropsychometry 